\title{
A STUDY OF PULSATION IN RR LYRAE MODELS*
}

\author{
ROBERT F. Christy \\ California Institute of Technology, Pasadena, California \\ Received August 5, 1965
}

\section{ABSTRACT}

Non-linear calculations of intermediate amplitude to investigate stability have been carried out for one hundred RR Lyrae models of varying composition, mass, luminosity, and effective temperature. Of these, the calculations have been extended to the maximum amplitude for fifty models. The dependence of the location of the instability strip on composition, mass, and luminosity has been explored. In particular, the high-temperature boundary of the strip increases by $500^{\circ} \mathrm{K}$ for each 15 per cent increase in the mass fraction of helium.

The dependence on effective temperature of the phase shift, the ratio of radius and luminosity amplitudes, and the shape of the light-curve has been explored, and the correlations of these three measures have been found. These measures may then be applied to observed variables to determine their location in the instability strip and the helium content of their envelopes. The examples studied give best agreement at about 30 per cent helium by mass.

The calculations reveal many details of the shapes of the light- and velocity-curves which may be compared to observation. A number of features of these curves of known variables are discussed, and the underlying explanation is investigated.

The sources of the driving energy of these models have been explored. In the models of the most favored composition, 30 per cent helium, the hydrogen ionization region is almost as important to the driving as is the He II ionization. The effects that cause the amplitude to cease growing and level off at the observed maximum amplitude are explored: they appear to involve several non-linear effects, particularly a steep velocity front, in the $\mathrm{He}$ II ionization zone.

A new kind of period-luminosity relation has been discovered. The shortest period at which the fundamental mode persists, before it changes to the first harmonic, has been found to be independent of mass and composition and to depend only on the luminosity. This relation was found to be $P_{\mathrm{tr}}=0057$ $(L / L \odot)^{0.6}$ days. This has been used to interpret the varying period distributions of RR Lyrae stars in clusters and in the field with the result that the luminosities are found to vary from $M_{\text {bol }}=0.57$ for very metal-weak clusters to $M_{\text {bol }}=0.96$ for clusters only slightly weak in metals. Metal-rich variables are slightly less luminous still. These results lead to the conclusion that the masses of RR Lyrae stars are near $0.5 M \odot$, which must imply a significant mass loss. These conclusions appear to be consistent with the possible interior models.

\section{INTRODUCTION}

\section{a) Background}

This paper attempts to explore pulsating stars by the numerical integration of the equations of motion. The methods used in this exploration have already been reported in "The Calculation of Stellar Pulsation" (Christy 1964a; hereinafter referred to as "Paper II)." The purpose of this exploration or survey is, of course, first to improve our understanding of the pulsation phenomenon; in addition, a principal object is to find relations and correlations between the observable features of pulsating stars and the physical properties of the models that reproduce those features. From this point of view, a pulsating star provides us with a great wealth of detailed observational material which may well lead us to a corresponding wealth of deduced knowledge of the physical properties of the star when we have learned how to properly interpret and reduce the observational material. This paper is intended to be a start toward interpreting the non-linear features of pulsation.

The program of learning the properties of pulsating stars through the study of pulsation was initiated by Eddington, who first calculated the period of such stars and thereby learned their mean densities by use of the relation $P$ (days) $\sqrt{ }(\rho / \rho \odot)=Q$ (the pulsation

* Supported in part by the Office of Naval Research Contract [Nonr-220(47)] and the National Aeronautics and Space Administration grant (NsG-426). 
constant). In addition, Eddington discussed in considerable detail the possible causes of pulsation but was not able to come to a definite conclusion.

The study of the linear pulsation theory was extended by others but it was Zhevakin who first found that the interaction of $\mathrm{He}$ II ionization with pulsation led to a sufficiently strong unstabilizing force that it could overcome the damping and drive pulsation. ${ }^{1}$ More detailed linear calculations by Cox (1963) and by Baker and Kippenhahn (1962) confirmed this result. Another important source of instability for driving pulsation, the influence of the hydrogen ionization, was found by the author (Christy 1962; hereinafter referred to as "Paper I") and elaborated in detail in Paper II in fully non-linear calculations.

However, although the linear theory has been able to lead to an understanding of pulsation, and to an approximate identification of the instability strip in the H-R diagram, it has not been possible (by that technique) to calculate correctly even the phase relations of the light- and velocity-curves or their relative amplitudes. No calculation of higher-order corrections to the linear theory has yet been made. As a result, the sole point of detailed contact between theory and observation remained the computation of the period.

The above inadequacies of the linearized theories have convinced the author that the attempt to construct a full non-linear theory is worthwhile. Such non-linear calculations should be able to give not only the correct periods but also the correct phase relations, the correct relative amplitudes of light- and velocity-curves, the correct temperature variations, and indeed the correct amplitudes themselves of all of these observable variables and the correct shapes in detail of the observed variations. Such a program, when successful, then offers a great variety of detailed comparisons with observation. This should permit the determination of whether the theory is accurate and, further, permit the determination of additional physical properties of the stars which can now only be assumed. The present survey, for the first time, attempts to provide this detailed basis of comparison of observation and calculation.

\section{b) Comparison with the Linear Theory}

As there have been several attempts to account for pulsation by means of the linear theory, it seems worthwhile to present a discussion of the points of similarity and difference between the non-linear treatment given in Paper II and the linear treatment.

\footnotetext{
${ }^{1}$ A review of much of Zhevakin's research is to be found in Zhevakin (1963) which gives references to earlier work. Although he was the first to point out the importance of He II ionization to Cepheid pulsation, he has also made extensive claims to an explanation of other variable stars ranging from $\beta$ Canis Majoris types to long-period variables. I have been unable to understand the basis of these claims and believe they result from an unwarranted extension of results obtained for a model simulating $\delta$ Cephei to other luminosities. It is not clear to me that the luminosity parameter $L_{0}$ and thereby the nonadiabatic parameter $y_{z}$ in his work can be varied without reconstructing the basic stellar model.

More recently Aleshin (1964a), working with Zhevakin, has initiated partly non-linear calculations of the ionization zones. His method bears some resemblance to the method I used in Paper II, but he uses linearized approximations to the equation of state and opacity and a sinusoidal driving at the lower boundary instead of studying freely developing oscillations of the whole star. Aleshin (1964b) has made some criticism of Paper I (Christy 1962) and contends that hydrogen plays no significant role in the pulsation. I have already commented in Paper II (Christy 1964a) on some correction and reduction of my estimates in Paper I. The treatment he uses is the linearized version which I discuss later and which I believe is basically incapable of providing a realistic estimate of the hydrogen zone. In addition, he and Zhevakin appear to use an incorrect opacity law in the atmospheric region $\left(T<10000^{\circ} \mathrm{K}\right)$. In fact, the actual opacity in this region decreases by many orders of magnitude between $10000^{\circ}$ and $5000^{\circ} \mathrm{K}$, whereas the data of his Table 1 shows an increase of opacity with decreasing temperature. It is only in view of the low opacity and relatively large mass of the atmospheric layers, as shown in Paper I, that they can play a significant role in pulsation.

Although, as discussed above, I disagree with some features of the methods used by Zhevakin, my principal contention is that the results of my calculations (see Paper II) agree in detail with observations on variable stars. It will be possible to understand better which features of my calculations should be improved when others have also achieved similar agreement with observation.
} 
It should first be emphasized that the linear treatment uses less computing machine time, and therefore, it would be desirable to explore the problems first by the linear method. It is unfortunate that a broad survey of this kind has not yet been carried out. In comparing the linear and non-linear calculations, we have first examined the nonlinear calculations to find in which region of the star the final amplitude must be considered large. In the region of $10^{5}{ }^{\circ}<T<4 \times 10^{5}{ }^{\circ}$, the amplitude is small, and the motion is almost completely adiabatic (all temperatures throughout are in degrees Kelvin). In the region $6 \times 10^{\circ}<T<10^{5}$, the amplitude is also quite small, essentially sinusoidal and quasi-adiabatic but dissipating. The region of $\mathrm{He}$ II ionization $(3 \times$ $10^{4}<T<6 \times 10^{4}$ ) already is significantly non-linear in its variation (see Fig. 1 ). However, the behavior of actual stars in this $\mathrm{He}$ II zone is not so non-linear that it is difficult to see the relation with the linear theory. It is likely that the actual behavior in the He II zone is only a modification of the behavior calculated in the linear theory. The region where it is very difficult to relate the linear and non-linear calculations is the region of $T<3 \times 10^{4}$ which I have called the hydrogen zone.

Recent linear calculations by Baker and Kippenhahn (1965) have attempted to include the hydrogen zone. They pointed out two particular peculiarities of this region when linear calculations are used. First, the behavior of this region is sensitive to the outer boundary conditions employed, about which there seems to have been some uncertainty. Second, the amplitude of, for example, the temperature variation in the hydrogen zone is so large that they doubt whether their results can have bearing on the actually observed unstable motions.

It is in relation to these two peculiarities that the non-linear theory can perhaps make new contributions. They both pertain to the surface regions of the star where the actual amplitudes are so large that it is indeed difficult to see how to make a linearization that does not completely falsify the real behavior. There appears to be no real difficulty in using the non-linear method to deal with external boundary conditions. As was done in Paper II, the pressure is taken to be zero at the stellar surface. It is also clear that the radiation should be treated by the complete time-dependent transport theory. However, the diffusion approximation introduced in Paper II seems to be an adequate treatment insofar as the principal physics of the pulsation is concerned, and it is unlikely that significant changes in the behavior of the pulsation would arise from a more exact radiation transport theory.

Regarding the temperature (and other) variations in the hydrogen zone, the nonlinear theory (and the observations) show that the description must be in terms of a moving front. Thus there is a relatively thin layer of the star in which the temperature rises from photospheric values to 20000 or $30000^{\circ}$. This steep temperature front then moves up and down through a considerable mass (compared to the conventional photospheric mass) of the star in the course of the cycle of pulsation. The front is also steeper when it is moving out and less steep when it recedes.

Now a careful examination of the results of the linear theory (Baker and Kippenhahn 1965) shows that they are not inconsistent with this picture. However, in the linear approximation, a steep moving front appears almost as a singularity in the amplitude that would approach infinity if the actual front were a step function. The out-of-phase terms in the amplitude show the motion of the front, but the actual amplitude of the variations in this region can only be seen from non-linear terms. Thus the linear theory shows an excessively large amplitude in a region of negligible thickness whereas the actual motion involves a large (but finite) amplitude in a region of considerable thickness. The significance of the hydrogen zone is related to how far the front actually moves and how much phase delay it can introduce into the light-curve, and these questions seem almost impossible to answer from the linear theory.

It is usually argued that the purpose of the linear theory is to investigate the initiation of motion in a static envelope. The use of the method is also questionable, however, 


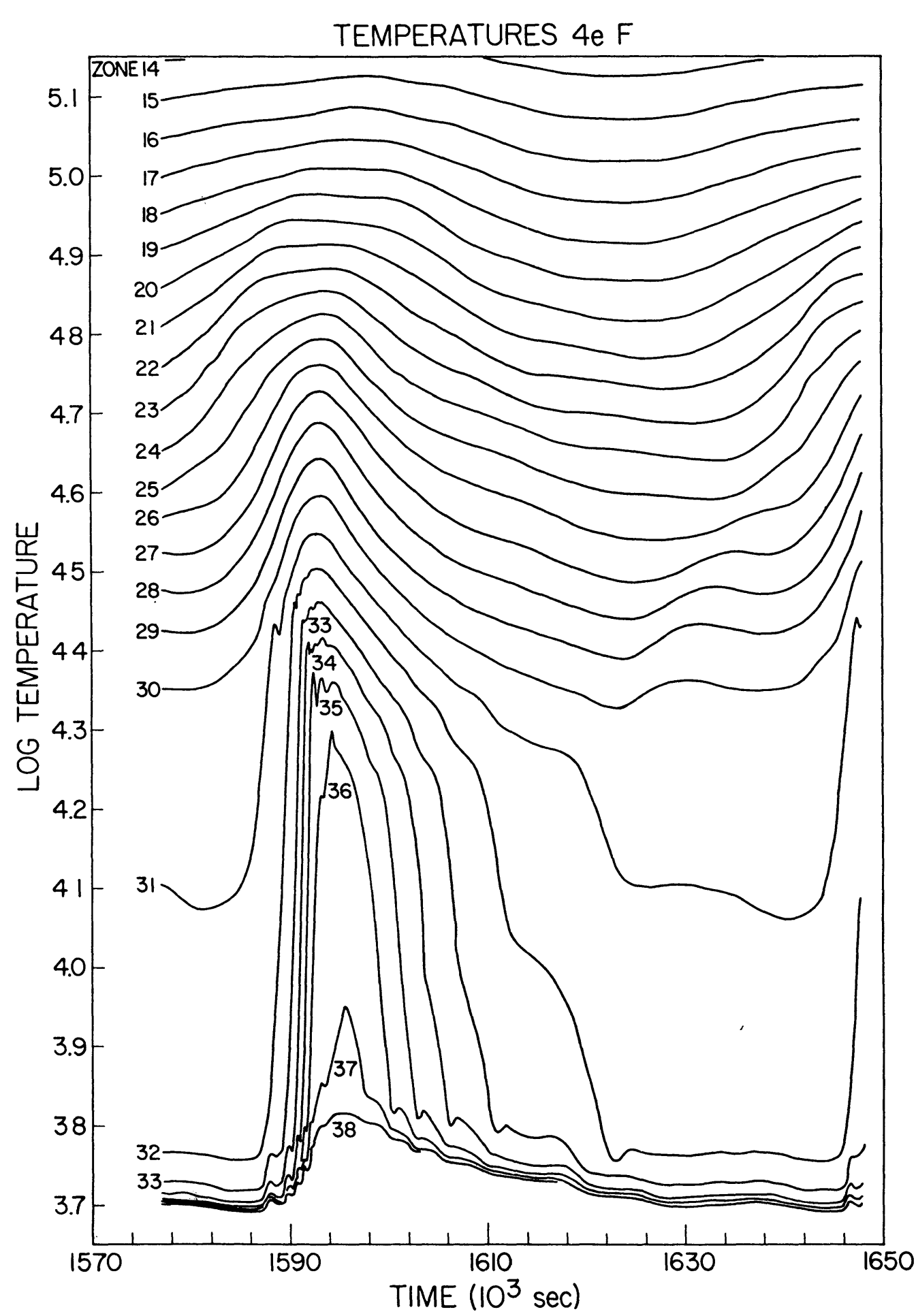

Fig. 1.-The temperature variation at various depths to $10^{\circ}{ }^{\circ} \mathrm{K}$ for model $4 \mathrm{e} F$ 
even for this purpose as the static envelope is also unstable to convective motions. It is customary to investigate the incipient instability to pulsation in an envelope already convecting. In many cases, however, the time for the growth of the pulsation is comparable to the time for growth of convection and it is unclear what should be taken as the static model. We thus realize that the growth of pulsation is basically a question of evolution-i.e., the pulsation must grow in models which are just evolving into the unstable region. Since evolution is much slower than the growth of pulsation, this means that pulsation will first appear at small amplitude only in models which are at the entering boundary of the region and are therefore marginally unstable. We will never find a star with infinitesimal amplitude in the middle of the unstable region.

The linear theory should then be sufficient to define the boundaries where stars are entering the unstable region. It is a peculiarity of non-linear methods that the boundaries where pulsating stars are leaving the unstable region may well differ from those for stars entering, as the region in the H-R diagram for maintaining pulsation in a star may be more extensive than the region where it can be initiated. In particular, at the low-temperature boundary where convection is so important, it is likely that pulsation and convection are competing modes for the instability in the hydrogen zone. Then we would expect that the presence of pulsation could inhibit convection and vice versa. The exploration of these interesting questions is still before us.

It appears from the non-linear calculations that it is just in the hydrogen zone that the characteristic shape of the light-curve and its phase shift and amplitude are determined. It is thus not surprising that these features have eluded the linear theory. In order to illustrate this behavior, we present in Figure 1 the time dependence of the temperature as a function of depth (zone number) in the star for problem $4 \mathrm{e} \mathrm{F}$ at its final full amplitude. It is already seen in Paper II (Fig. 7), that the velocity-curve is much less sensitive to the hydrogen zone than the light-curve. Correspondingly, as the accurate treatment of the heat flow in the hydrogen zone is one of the major difficulties of the non-linear calculations, we find that the calculation of the velocity-curve is much more reliable than present calculations of the light-curve. It is unfortunate that precisely the reverse is true of the observations.

The results of the non-linear calculations concerning the significance of the hydrogen zone have persistently surprised those familiar with the linear calculations because, in the static stellar model, the mass of gas lying above the hydrogen ionization region (just the photosphere) was too small to provide a significant phase delay. However, it is the mass of un-ionized hydrogen (the depth of the photosphere) at the temperature and luminosity minimum that is important. Because of the great sensitivity of the opacity to temperature, the photosphere is several times as deep as minimum temperature and pressure as in the static model and (as was shown in Paper I) is then able to provide a quite significant phase delay.

As a result of these considerations, the author has come to the view that the linear theory is probably reliable as regards those features associated with the He II zone and deeper zones in the star but that it is unlikely that a linear calculation will be able to represent correctly those features associated with the hydrogen zone. It was pointed out in Paper I and shown in Paper II that the hydrogen zone is also a very significant contributor to the instability and the driving of the pulsation. In the same way, then, it is not to be expected that the linear theory can accurately account for the location and width of the unstable region in the H-R diagram.

\section{c) The Method of Attack}

The general procedure of initiating, in the calculation, some approximation to a pure periodic pulsation has been described in Paper II. It consists in superimposing some prechosen velocity distribution, as a function of $R$, the radius, on a static model envelope, and following in time the development of the motion. It was soon found, however, that 
the kind of results that could be obtained and the approach depended on the particular stellar model.

The most significant parameter in studying a model of definite $T_{e}, X / Y$, and period is the mass or luminosity. The same period can be found for a wide range of masses with appropriate radii and luminosities. In general, it was found that the growth or decay of the amplitude was fast in models of low mass and exceedingly slow in models of large mass. The kinetic energy of the envelope of a model of large mass is very much greater than for one of low mass and changes much more slowly in time. In this connection, masses much larger than $1 M \odot$ are difficult to treat for RR Lyrae periods, whereas for a period of 5 days as much as $5 M \odot$ can be treated by this method.

Now this method of exploring pulsation determines the existence of stability or instability and obtains many other detailed results on pulsation by following the motion in time. If the amplitude changes by as little as $10^{-3}$ per period, then it becomes difficult to determine the question of instability. The principal problem is not the slow growth (or decay) of the fundamental mode but the inability to eliminate the first and higher harmonics from the motion in a reasonable time (1 hour) on a computer.

The problem of determining when a non-periodic motion is growing or decaying is quite difficult. The method adopted here was to examine the peak kinetic energy of the envelope each period and find the difference for 3 periods (to minimize effects of the first harmonic). This measure was reasonably insensitive to harmonic mixture.

This feature of the problem has led to two different types of survey. An instability survey was aimed at extracting the maximum amount of information from the computed behavior of models at about $\frac{1}{2}$ maximum amplitude where there was a considerable mixture of higher unwanted harmonics. These runs were followed on the computer for about 8 periods (usually 900 time steps and about $7 \mathrm{~min}$ on an IBM 7094). Under these circumstances, it was found that the principal effects of the first harmonic on the fundamental could be eliminated by averaging over three consecutive fundamental periods or four first harmonic periods since the ratio of periods is near $\frac{3}{4}$. This averaging permitted approximate measures to be extracted for all amplitude-insensitive gross features of the motion. These features included the question of stability or instability, the period, the phase lag of the luminosity relative to the velocity, the ratio of radius amplitude (or velocity amplitude) to light amplitude.

The second survey was aimed at exploring the computed behavior of envelope models at their maximum amplitude. For the less massive models and for the first-harmonic models of any mass, the kinetic energy of the envelope is sufficiently small that the growth or decay of the amplitude takes 20 or 30 periods which can readily be followed on the computer. At the same time, as the dominant mode grows in amplitude, the other modes decay so that after some 30 periods, a nearly pure mode is present and it is oscillating within 5 to 10 per cent of its maximum amplitude. Under these conditions, the motion has also developed nearly all the peculiar non-linear features that characterize the final steady periodic motion. The procedure for extrapolating the behavior so as to find the maximum amplitude was discussed in Paper II. It is then possible to obtain all details such as amplitudes and shapes of computed curves in order to compare them with observed ones. In addition, it is possible to "see" inside the star and study the physical processes taking place.

For the more massive models, the above procedure failed because of the slow approach to the asymptotic behavior. However, new numerical techniques were evolved for accelerating the approach to maximum amplitude. Some models were started at relatively large amplitude. Some were initiated at greater than final amplitude. These procedures, however, both introduce large amplitudes of the unwanted first harmonic which is then very slow to decay. Strong shock waves with associated "flashes" of light and strong dissipation appear when the amplitude is too large. A more successful method in these cases was to initiate a small-amplitude relatively pure harmonic and then to 
slowly augment its amplitude artificially. In between such periods of artificial growth, the free motion is studied to find if it is slowly growing or decaying. The motion is finally followed on the computer when it is near its maximum and the details of the motion are again studied.

In the course of these many different approaches to the asymptotic amplitude, some from below, some from above, and some artificial, it has become quite clear that the final motion, with the exception below, is quite independent of the initial conditions and also of the approach to the maximum amplitude. The only exception found consists of cases which are discussed later where the envelope is able to pulsate steadily in either the first harmonic or in the fundamental mode, depending on its initial conditions.

\section{d) Limitations of the Method}

One limitation of the method has already been mentioned: the difficulty of studying the more massive models. Thus, in studying models of period about $\frac{2}{3}$ day, the most massive which was examined was $1.3 \mathrm{M} \odot$ and even for this mass the growth or decay rates were quite slow (about 1000 periods). It would be quite difficult to use this method to explore the stability of much more massive models with this period. This limitation on mass is of course closely related to the luminosity and period, and for a period of, e.g., 5.4 days ( $\delta$ Cephei) a mass of $3.2 M \odot$ was found to have a rapidly growing amplitude. In fact, in no case where a model seemed to correspond closely to observed variables was the growth or decay rate of the amplitude found to be so slow that the method could not be used.

Another difficulty with the method has been discussed at some length in Paper II. The treatment of heat flow in the region of hydrogen ionization $\left(10^{4}{ }^{\circ}\right)$ is quite difficult, and the best compromise found so far still leads to temperature- and luminosity-curves having a fine structure associated with the cooling of successive zones through this temperature. In the models on the high-temperature side of the instability strip, it was convenient to make the zones of sufficiently small mass that this difficulty was of no consequence. However, in attempting to explore the low-temperature boundary of the instability, this limitation became severe. There, it was not at all convenient to achieve such fine zoning and, in fact, the difficulty in correctly treating the hydrogen ionization zone for such models leaves still unclear where the low-temperature boundary should be. It is noteworthy, as was pointed out in Paper II, that just for these same models the contribution of convection to the heat flow in the hydrogen zone can no longer be ignored. It would seem that this boundary of the instability can be correctly explored only by including the convection in the hydrogen zone.

\section{THE INSTABILITY REGION}

a) The Static Models

The dynamic models investigated in this program were initiated from static models that were obtained as the first step of the calculation. The region of the envelope that is relevant to this problem $\left(T<4 \times 10^{5}\right)$ is sufficiently remote from the dense stellar core that the pulsation behavior of the model is independent of the central conditions. This meant that it was possible to use an envelope model that was integrated inward from the photosphere and was terminated before reaching the center so that it ignored the central boundary conditions. The depth of the envelope was chosen so that the estimated sound travel time through the remaining core was less than 1 per cent of the total sound travel time through the envelope. This usually involved an envelope mass of about 2 per cent of the stellar mass and a temperature at its base somewhat in excess of $10^{6}{ }^{\circ}$. The radius of the base of the envelope was never greater than $\frac{1}{7}$ of the stellar radius. As a result, models were explored for a variety of masses at a given luminosity, effective temperature, and composition. Actual giants also take advantage of the possibilities of 
an inhomogeneous structure to appear with different masses at the same luminosity. Since the region of the envelope that is particularly relevant to these models involves less than 1 per cent of the stellar mass, it was reasonable to ignore composition inhomogeneities in the models.

Even then, a considerable number of physical parameters are required to describe an envelope. I have used the following set of parameters: mass $M$ (of star), mean luminosity $L_{0}$, effective temperature $T_{e}$ (of static model-equivalent to choice of mean radius $R$ ), hydrogen-helium ratio (by mass). An additional possible parameter, $Z$, the "metal" content has been ignored in this survey for the following reasons. The metals are only of significance in the opacity, not in the equation of state. In the opacity, the metals are important for $T>2 \times 10^{5}$, where they become the dominant contribution to the opacity. However, the pulsation motions are quite negligible in this region and the opacity there can have no effect. In the other limit, the metals are significant in determining the electron pressure and thereby the opacity for $T<5000^{\circ}$. However, the RR Lyrae and even the Cepheid models have atmospheres hot enough (and of low density) so that the metal contributions to the photospheric opacity are also quite small. It is thus reasonable to ignore the metal content in such a survey. In addition, of course, cluster-type RR Lyrae variables are low in metals. The survey was carried out with $Z=0.002$ (by mass). A series of static models with $Z$ ranging up to 0.08 was examined and confirmed the argument given above that the effect of the metal content on the envelope model is inconsequential.

The remaining four parameters still require a tremendous number of models for adequate coverage. It was for this reason that the survey was limited to RR Lyrae variables. For these purposes, they were defined as variables of period in the range $\frac{1}{4}-1$ day. This period restriction then permitted a rough relation between mass and luminosity to be introduced. The survey attempted to cover all pulsating model envelopes with $Z=$ 0.002 and with periods in the range (approx.) $\frac{1}{4}-1$ day.

The zone structure of these static models was made to parallel closely the treatment in Paper II. About three zones were used to describe the region above the photosphere. The next five zones comprised the region of strong driving from hydrogen ionization. The next five zones were the region of strong driving by the He II ionization. The next ten zones contributed the principal damping to the motion. A further ten zones behaved almost adiabatically. The final five zones, terminating at about $\frac{1}{10}$ of the outer radius of the star, were of no real consequence in the calculation but were introduced to remove the somewhat crude interior boundary condition (zero motion) farther from the active region of the star.

\section{b) The Instability Survey}

A preliminary survey was carried out to find the unstable region and to find the general characteristics of self-sustaining pulsations. This survey was briefly reported (Christy $1963 a, b$ ) and provided the basis of much more extensive investigations reported here.

The present series of models can be conveniently divided into a number of sequences. In each sequence, the mass, luminosity, and composition were kept fixed and the stability and properties of pulsation were investigated for a series of different mean effective temperatures $\left(T_{e}\right)$ or radii $R$.

No entirely satisfactory precise observable definition of $T_{e}$ or $R$ as used here for a pulsating star has been found. The values here refer to the static-envelope model before the initiation of pulsation. In general, the fractional radius change is small so that the radius as used here is within 1 per cent of the average between the actual maximum and minimum radii of the pulsating star. This mean radius, combined with the time-average luminosity $L_{0}$, can then be used to define a mean $T_{e}$.

In each sequence, the high $T_{e}$ side of the instability strip was studied and in most cases 
the sequence extended to a low $T_{e}$ near the low $T_{e}$ boundary of instability. As was discussed in $\S I$ and in Paper II, it was not possible to find precisely the low $T_{e}$ boundary. The various sequences are listed in Table 1 , where the boundaries in $T_{e}$ for various motions are also listed.

The compositions studied were (by mass) 0 per cent $\mathrm{He}, 15$ per cent $\mathrm{He}, 30$ per cent $\mathrm{He}, 45$ per cent $\mathrm{He}$, and 60 per cent $\mathrm{He}$, all with a content of 0.2 per cent of elements other than hydrogen and helium. The most extensive set of masses-approximately $0.166,0.25,0.376,0.58,0.88$, and $1.33 M \odot$-was investigated for 45 per cent He.

Most of the calculations were carried out for $L_{0}=1.50 \times 10^{35} \mathrm{ergs} / \mathrm{sec}$ (bolometric). Assuming no bolometric correction, this corresponds to $M_{v} \approx+0.76$. Some calculations were carried out for $L_{0}=2.00 \times 10^{35} \mathrm{ergs} / \mathrm{sec}\left(M_{v} \approx+0.45\right)$ and some for $L_{0}=2.80 \times$ $10^{35} \mathrm{ergs} / \mathrm{sec}\left(M_{v} \approx+0.08\right)$.

This survey was aimed first at defining the region of instability-i.e., the boundaries of instability in the H-R diagram-and relating it if possible to the properties of the static models. In this way, it may be possible to define the unstable region in terms of the properties of the static stars at the boundaries.

The results of the survey are included in Table 2, which is arranged in sequences according to helium content, luminosity, and mass. The first column provides a reference number and also designates whether it is the fundamental mode $F$ or the first harmonic $H$ that is being studied. Those models which were calculated at maximum amplitude are denoted by an asterisk; they are the most accurate. The next three columns give the $T_{e}, R$, and surface gravity, $g$, computed for the static model.

In the fifth column, I have presented an estimate of the maximum fraction of the flux that could be carried by convection in the hydrogen convection zone. All these models are violently, convectively unstable, but, as has been discussed in Papers I and II, since these envelopes are of such low density the maximum flux that could be carried by convection involving subsonic velocities is still small compared to the actual flux. This maximum convective flux has been estimated from the expression

$$
H_{c} \approx 10^{6} p \mathrm{ergs} / \mathrm{cm}^{2} \mathrm{sec}
$$

(Paper I, p. 889), where $p$ is the pressure in dynes $/ \mathrm{cm}^{2}$. For each static model, the ratio of $H_{c} \times 4 \pi R^{2}$ to $L_{0}$ was computed and is given in the fifth column. The actual calculations assumed that all the flux is carried by radiation alone. The period $P$ (in days) and the resulting pulsation constant $Q=P \sqrt{ }(\rho / \rho \odot)$ are given in the next two columns. The period given here was found by evaluating the elapsed time for as many complete cycles as feasible and dividing by the number of cycles. We have found no clear evidence for a change of period in the amplitude range studied from $\Delta R / R \approx 0.05$ to 0.15 . We concluded that the period is remarkably insensitive to amplitude, and the period we find for intermediate or large amplitude should be almost identical with that computed in the linearized theory at infinitesimal amplitude.

In Paper I (p. 891) and Paper II (p. 568) it was pointed out that the excess heat capacity associated with ionizing the surface layers of pulsating stars is closely related to the phase lag and the efficiency of operation of the driving mechanism. For this reason, the eighth column shows the time required (in the static model) to ionize those surface layers that are incompletely ionized (including both the helium and hydrogen ionization transitions) if the mean luminous flux of the star were entirely devoted to that process. For reference, the time is divided by the period of the fundamental oscillation so that the resulting quantity in the eighth column is some measure of the phase lag that could be generated in the ionizing layers. Actually, the measure of the possible phase lag due to He II ionization is fairly good. This calculation does not give a satisfactory estimate of the phase contributed by hydrogen ionization, as the condition of the atmosphere just prior to the light maximum is one in which the temperature is low and the ionization 
TABLE 1. The Instability Region

\begin{tabular}{|c|c|c|c|c|c|c|c|c|c|c|c|c|c|}
\hline & Sequence & Figure & $\begin{array}{l}\$ \\
\text { Helium } \\
\text { by Mass }\end{array}$ & $\begin{array}{l}\text { Luminosity } \\
\left(10^{35}\right. \\
\text { erg } / \mathrm{sec})\end{array}$ & $\left(10^{33} \mathrm{gm}\right)$ & $\begin{array}{l}\text { High }_{\mathrm{e}} \\
\text { limit, } \\
\mathrm{F} \text { or } \mathrm{H}\end{array}$ & $\begin{array}{c}\text { Transition } \\
\mathrm{T}_{\mathrm{e}} \begin{array}{l}\text { Between } \\
\mathrm{F}, \mathrm{H}\end{array}\end{array}$ & $\begin{array}{l}\text { Low } \mathrm{T}_{\mathrm{e}} \\
\text { Limit } \\
\mathrm{F} \text { or } \mathrm{H}\end{array}$ & $\begin{array}{l}\text { Ion Time } / \mathrm{P}_{\mathrm{F}} \\
\text { at High } \mathrm{T}_{\mathrm{e}} \\
\text { Limit }\end{array}$ & $\begin{array}{l}\mathrm{K} \text { at } \\
\text { High } \mathrm{T} \\
\text { Limit }\end{array}$ & $\begin{array}{c}P_{F} \text { at } \\
\text { Transition } \\
\text { (Days) }\end{array}$ & $\begin{array}{c}Q \text { at } \\
\text { Transition }\end{array}$ & ${ }_{\text {Transition }}^{\nabla} .83^{\text {at }}$ \\
\hline \multirow{17}{*}{$\Xi$} & 1 & -- & 0 & 1.50 & 0.75 & $\sim 6000 a_{F}$ & --- & $<5800^{\circ} \mathrm{F}$ & 0.10 & 1.57 & & & \\
\hline & 2 & 2 & 15 & 1.50 & 0.75 & $6500^{\circ} \mathrm{F}$ &.- & $<6000^{\circ} \mathrm{F}$ & 0.13 & 1.84 & & & \\
\hline & 3 & 2 & 15 & 1.50 & 1.15 & $6650^{\circ} \mathrm{H}$ & $6500^{\circ}$ & $<6200^{\circ} \mathrm{F}$ & 0.14 & 1.67 & 0.52 & 0.0362 & 19.1 \\
\hline & 4 & 3 & 30 & 1.50 & 0.75 & $6900^{\circ} \mathrm{F}$ & -- & $<6000^{\circ} \mathrm{F}$ & 0.19 & 1.87 & & & \\
\hline & 5 & 3 & 30 & 1.50 & 1.15 & $7150^{\circ} \mathrm{H}$ & $6550^{\circ}$ & $\sim 5200^{\circ} \mathrm{F}$ & 0.18 & 1.81 & 0.51 & 0.0363 & 19.2 \\
\hline & 6 & 4 & 45 & 1.50 & 0.33 & $6950^{\circ} \mathrm{F}$ & -.- & $\leq 5200^{\circ} \mathrm{F}$ & 0.18 & 2.10 & & & \\
\hline & 7 & 4 & 45 & 1.50 & 0.50 & $7150^{\circ} \mathrm{F}$ & --- & $<5800^{\circ} \mathrm{F}$ & 0.19 & 1.97 & & & \\
\hline & 8 & 4 & 45 & 1.50 & 0.75 & $7500^{\circ} \mathrm{H}$ & $7100^{\circ}$ & $\leq 5500^{\circ} \mathrm{F}$ & 0.19 & 2.04 & 0.50 & 0.0370 & 18.8 \\
\hline & 9 & 4 & 45 & 1.50 & 1.15 & $7600^{\circ} \mathrm{H}$ & $6550^{\circ}$ & $\leq 5800^{\circ} \mathrm{F}$ & 0.21 & 1.75 & 0.51 & 0.0364 & 19.2 \\
\hline & 10 & 4 & 45 & 1.50 & 1.75 & $7150^{\circ} \mathrm{H}$ & --- & $6000^{\circ} \mathrm{H}$ & 0.32 & 1.05 & & & \\
\hline & 11 & 4 & 45 & 1.50 & 2.65 & stable & --- & --- & --- & --- & & & \\
\hline & 12 & 5 & 45 & 2.80 & 0.75 & $6950^{\circ} \mathrm{F}$ & --- & $<6200^{\circ} \mathrm{F}$ & 0.20 & 1.90 & & & \\
\hline & 13 & 5 & 45 & 2.80 & 1.15 & $7330^{\circ} \mathrm{H}$ & $6800^{\circ}$ & $<6200^{\circ} \mathrm{F}$ & 0.19 & 1.94 & 0.74 & 0.0370 & 18.8 \\
\hline & 14 & 5 & 45 & 2.80 & 1.75 & $7320^{\circ} \mathrm{H}$ & $6300^{\circ}$ & $\leq 5800^{\circ} \mathrm{F}$ & 0.21 & 1.57 & 0.74 & 0.0364 & 19.2 \\
\hline & 15 & 6 & 60 & 1.50 & 0.75 & $7920^{\circ} \mathrm{H}$ & $7100^{\circ}$ & $\sim 5600^{\circ} \mathrm{F}$ & 0.22 & 1.76 & 0.50 & 0.0371 & 18.7 \\
\hline & 16 & 6 & 60 & 1.50 & 1.15 & $7800^{\circ} \mathrm{H}$ & $6500^{\circ}$ & $<6000^{\circ} \mathrm{F}$ & 0.27 & 1.31 & 0.52 & 0.0363 & 18.9 \\
\hline & 17 & 7 & 60 & 2.00 & 0.75 & $7800^{\circ} \mathrm{H}$ & $7200^{\circ}$ & $<6000^{\circ} \mathrm{F}$ & 0.20 & 1.87 & 0.61 & 0.0374 & 18.5 \\
\hline
\end{tabular}


TABLE 2. The Instability Survey

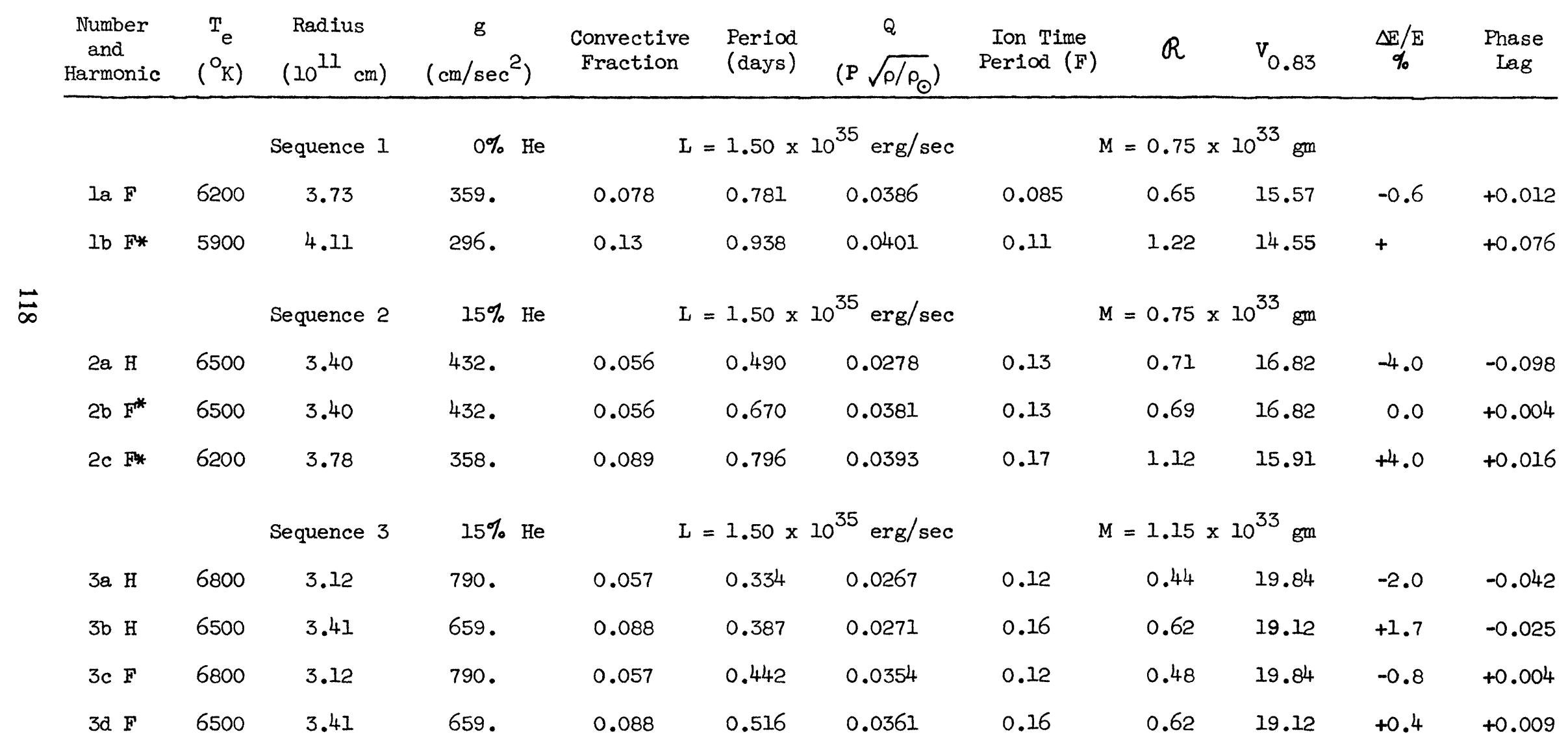


TABLE 2 (cont.)

\begin{tabular}{|c|c|c|c|c|c|c|c|c|c|c|c|}
\hline $\begin{array}{l}\text { Number } \\
\text { and } \\
\text { Harmonic }\end{array}$ & $\begin{array}{c}\mathrm{T}_{\mathrm{e}} \\
\left({ }^{\mathrm{O}} \mathrm{K}\right)\end{array}$ & $\begin{array}{l}\text { Radius } \\
\left(10^{11} \mathrm{~cm}\right)\end{array}$ & $\begin{array}{c}\mathrm{g} \\
\left(\mathrm{cm} / \mathrm{sec}^{2}\right)\end{array}$ & $\begin{array}{c}\text { Convective } \\
\text { Fraction }\end{array}$ & $\begin{array}{l}\text { Period } \\
\text { (days) }\end{array}$ & $\frac{Q}{\left(P \sqrt{\rho / \rho_{\odot}}\right)}$ & $\begin{array}{l}\text { Ion Time } \\
\text { Period (F) }\end{array}$ & $R$ & $v_{0.83}$ & $\frac{\Delta E}{\delta} / E$ & $\begin{array}{c}\text { Phase } \\
\text { Lag }\end{array}$ \\
\hline
\end{tabular}

Sequence $4 \quad 30 \% \mathrm{He} \quad \mathrm{L}=1.50 \times 10^{35} \mathrm{erg} / \mathrm{sec}$

$\begin{array}{lll}4 \mathrm{a} \mathrm{H} & 7100 & 2.86 \\ 4 \mathrm{bH} & 6800 & 3.11 \\ 4 \mathrm{c} \mathrm{F} & 7100 & 2.86 \\ 4 \mathrm{~d} \mathrm{F*} & 6800 & 3.11 \\ 4 \mathrm{eF*} & 6500 & 3.41 \\ 4 \mathrm{fF}^{*} & 6200 & 3.74\end{array}$

613.

516.

613.

516.

431.

0.373

0.0275

0.433

0.0281

0.496

0.0366

0.578

0.0375

0.678

0.0384

357.

0.802

0.0395

$L=1.50 \times 10^{35} \mathrm{erg} / \mathrm{sec}$ 5a $\mathrm{H} \quad 7400$

2.64

$5 \mathrm{~b} \mathrm{H} \quad 7100$

2.86

$5 \mathrm{c} \mathrm{H} * \quad 6800$

3.12

$5 \mathrm{~d} H * \quad 6500$

3.42

5 e $H^{*} \quad 6200$

3.75

5f F 6800

3.12

$5 g . F^{*} \quad 6500$

3.42

$5 h F * 6200$

3.75

$51 \mathrm{~F}^{*} 5900$

4.14

$5 j \mathrm{~F}^{*} \quad 5600$
$30 \%$

1104.

936.

0.027

0.254

0.0261

0.291

0.0265

788.

0.042

0.336

0.0269

0.389

0.0272

0.456

0.0277

0.444

0.0356

0.520

0.0364

0.612

0.0371

0.727

0.868

0.0389

545.

0.26

364.

0.46
0.0380
0.027

$M=0.75 \times 10^{33} \mathrm{gm}$

0.16

$0.87 \quad 18.69$

$-3.0 \quad-0.16$

0.20

$1.08 \quad 17.95$

$-0.5$

$-0.14$

0.16

$0.60 \quad 18.69$

$-2.0$

$-0.011$

0.20

0.83

17.95

$+1.0$

$+0.001$

0.25

1.10

17.15

0.31

1.54

16.24

$+$

$-0.002$

$+0.052$

$M=1.15 \times 10^{33} \mathrm{gm}$

0.15

0.50

20.81

$-1.5$

$-0.13$

0.65

20.42

$+0.2$

$-0.105$

0.89

19.86

$+1.6$

$-0.076$

$1.20 \quad 19.24$

$+3.0$

$-0.061$

2.03

18.51

$+3.0 \quad-0.005$

$0.74 \quad 19.86$

$+0.1$

$1.00 \quad 19.24$

$+1.0$

$+0.017$

0.41

1.36

18.51

$+1.7$

$+0.050$

0.54

1.66

17.74

$+2$.

$+0.08$

0.75

16.78

$+1.6$

$+0.16$ 
TABIE 2 (cont.)

\begin{tabular}{|c|c|c|c|c|c|c|c|c|c|c|c|}
\hline $\begin{array}{l}\text { Number } \\
\text { and } \\
\text { Harmonic }\end{array}$ & $\begin{array}{c}\mathrm{T}_{\mathrm{e}} \\
\left({ }^{\mathrm{O}} \mathrm{K}\right)\end{array}$ & $\begin{array}{l}\text { Radius } \\
\left(10^{11} \mathrm{~cm}\right)\end{array}$ & $\begin{array}{c}\mathrm{g} \\
\left(\mathrm{cm} / \mathrm{sec}^{2}\right) \\
\end{array}$ & $\begin{array}{l}\text { Convective } \\
\text { Fraction }\end{array}$ & $\begin{array}{l}\text { Period } \\
\text { (days) }\end{array}$ & $\begin{array}{c}Q \\
\left(P \sqrt{\rho / \rho_{\odot}}\right)\end{array}$ & $\begin{array}{l}\text { Ion Time } \\
\text { Period }(\mathrm{F})\end{array}$ & $R$ & $\mathrm{v}_{0.83}$ & $\underset{\&}{\Delta E / E}$ & $\begin{array}{l}\text { Phase } \\
\text { Lag }\end{array}$ \\
\hline & & Sequence 6 & $45 \% \mathrm{He}$ & \multicolumn{3}{|c|}{$L=1.50 \times 10^{35} \mathrm{erg} / \mathrm{sec}$} & \multicolumn{3}{|c|}{$M=0.33 \times 10^{33} \mathrm{gm}$} & & \\
\hline $6 a F$ & 7100 & 2.83 & 275. & 0.020 & 0.850 & 0.0422 & 0.16 & 0.99 & 14.35 & -9.0 & -0.022 \\
\hline $6 \mathrm{~b} F$ & 6800 & 3.08 & 231. & 0.032 & 1.000 & 0.0437 & 0.19 & 1.15 & 13.55 & +8 & +0.035 \\
\hline $6 c F$ & 5600 & 4.52 & 108. & 0.19 & 2.147 & 0.0529 & 0.49 & 1.70 & 10.08 & +30 & +0.21 \\
\hline $6 \mathrm{~d} F *$ & 5300 & 5.06 & 86. & 0.33 & 2.73 & 0.0568 & 0.66 & 1.61 & 9.00 & +20 & +0.25 \\
\hline & & Sequence 7 & $45 \% \mathrm{He}$ & \multicolumn{3}{|c|}{$L=1.50 \times 10^{35} \mathrm{erg} / \mathrm{sec}$} & \multicolumn{3}{|c|}{$M=0.50 \times 10^{33} \mathrm{gm}$} & & \\
\hline $7 \mathrm{a} \mathrm{H}$ & 7700 & 2.43 & 565. & 0.013 & 0.366 & 0.0281 & 0.14 & 1.01 & 18.03 & -7.1 & -0.15 \\
\hline $\mathrm{Tb} \mathrm{H}$ & 7400 & 2.63 & 484 . & 0.019 & 0.417 & 0.0285 & 0.17 & 1.60 & 17.36 & -2.9 & -0.072 \\
\hline $7 c F$ & 7400 & 2.63 & 484. & 0.019 & 0.559 & 0.0383 & 0.17 & 0.77 & 17.36 & -5.2 & -0.089 \\
\hline $7 d F *$ & 7100 & 2.85 & 411. & 0.024 & 0.647 & 0.0391 & 0.20 & 0.98 & 16.73 & +0.8 & -0.035 \\
\hline $7 e F$ & 6800 & 3.11 & 346. & 0.037 & 0.756 & 0.0402 & 0.25 & 1.18 & 15.96 & +6.0 & +0.051 \\
\hline $7 f \mathrm{~F}$ & 6500 & 3.40 & 289. & 0.066 & 0.898 & 0.0417 & 0.32 & 1.33 & 15.03 & +10 & +0.14 \\
\hline $7 g F$ & 6200 & 3.73 & 239. & 0.094 & 1.08 & 0.0436 & 0.39 & 1.67 & 14.20 & +15 . & +0.18 \\
\hline 7h F & 5900 & 4.12 & 196. & 0.16 & 1.31 & 0.0455 & 0.50 & 1.59 & 13.25 & +19 & +0.14 \\
\hline
\end{tabular}


TABIE 2 (cont.)

\begin{tabular}{|c|c|c|c|c|c|c|c|c|c|c|c|}
\hline $\begin{array}{c}\begin{array}{c}\text { Number } \\
\text { and } \\
\text { Harmonic }\end{array} \\
\end{array}$ & $\begin{array}{c}\mathrm{T}_{\mathrm{e}} \\
\left({ }^{\mathrm{O}} \mathrm{K}\right)\end{array}$ & $\begin{array}{l}\text { Radius } \\
\left(10^{11} \mathrm{~cm}\right)\end{array}$ & $\begin{array}{c}\mathrm{g} \\
\left(\mathrm{cm} / \mathrm{sec}^{2}\right) \\
\end{array}$ & $\begin{array}{l}\text { Convective } \\
\text { Fraction }\end{array}$ & $\begin{array}{l}\text { Period } \\
\text { (days) }\end{array}$ & $\begin{array}{c}Q \\
\left(P \sqrt{\rho / \rho_{\odot}}\right)\end{array}$ & $\begin{array}{l}\text { Ion Time } \\
\text { Period }(F)\end{array}$ & $R$ & $\mathrm{v}_{0.83}$ & $\frac{\Delta E}{\phi} / E$ & $\begin{array}{l}\text { Phase } \\
\text { Lag }\end{array}$ \\
\hline & & Sequence 8 & $45 \% \mathrm{He}$ & \multicolumn{3}{|c|}{$L=1.50 \times 10^{35} \mathrm{erg} / \mathrm{sec}$} & \multicolumn{4}{|c|}{$M=0.75 \times 10^{33} \mathrm{gm}$} & \\
\hline $8 \mathrm{aH}$ & 7700 & 2.43 & 845. & 0.015 & 0.285 & 0.0268 & 0.17 & 0.77 & 19.83 & -2.0 & -0.12 \\
\hline $8 \mathrm{~b} \mathrm{H*}$ & 7400 & 2.63 & 721. & 0.022 & 0.326 & 0.0272 & 0.20 & 1.04 & 19.39 & +1.0 & -0.100 \\
\hline $8 \mathrm{c} \mathrm{H} *$ & 7100 & 2.86 & 611. & 0.033 & 0.375 & 0.0276 & 0.25 & 1.23 & 18.84 & + & -0.105 \\
\hline $8 d F^{*}$ & 7100 & 2.86 & 611. & 0.033 & 0.502 & 0.0370 & 0.25 & 0.89 & 18.84 & +0.6 & +0.009 \\
\hline $8 e F^{*}$ & 6800 & 3.12 & 515. & 0.052 & 0.582 & 0.0377 & 0.30 & 1.15 & 18.18 & +2.5 & +0.039 \\
\hline $8 f F^{*}$ & 6500 & 3.41 & 430. & 0.082 & 0.680 & 0.0385 & 0.39 & 1.38 & 17.44 & +3.9 & +0.066 \\
\hline $8 g F^{*}$ & 6200 & 3.75 & 357. & 0.13 & 0.802 & 0.0394 & 0.49 & 1.71 & 16.62 & +3.5 & +0.133 \\
\hline \multirow[t]{3}{*}{ ב } & 5900 & 4.14 & 292. & 0.20 & 0.957 & 0.0406 & 0.64 & 1.79 & 15.72 & + & +0.197 \\
\hline & 5600 & 4.58 & 238 . & 0.33 & 1.157 & 0.0421 & 0.98 & 1.40 & 14.52 & + & +0.249 \\
\hline & \multicolumn{2}{|r|}{ Sequence 9} & $45 \% \mathrm{He}$ & \multicolumn{3}{|c|}{$L=1.50 \times 10^{35} \mathrm{erg} / \mathrm{sec}$} & \multicolumn{4}{|c|}{$M=1.15 \times 10^{33} \mathrm{gm}$} & \\
\hline $9 \mathrm{a} \mathrm{H}$ & 7700 & 2.44 & 1293. & 0.023 & 0.223 & 0.0259 & 0.20 & 0.55 & 20.69 & -0.4 & \\
\hline $9 \mathrm{~b} \mathrm{H}^{*}$ & 7400 & 2.64 & 1103. & 0.032 & 0.254 & 0.0262 & 0.24 & 0.77 & 20.44 & +0.7 & -0.079 \\
\hline $9 \mathrm{cH} *$ & 7100 & 2.87 & 935 & 0.048 & 0.290 & 0.0264 & 0.30 & 1.04 & 20.07 & +1 & -0.067 \\
\hline $9 \mathrm{~d} \mathrm{H}^{*}$ & 6800 & 3.12 & 787. & 0.075 & 0.335 & 0.0267 & 0.38 & 1.44 & 19.71 & + & -0.043 \\
\hline $9 e H^{*}$ & 6500 & 3.42 & 657. & 0.12 & 0.387 & 0.0271 & 0.49 & 1.96 & 19.19 & +1 & -0.022 \\
\hline $9 f F^{*}$ & 6500 & 3.42 & 657. & 0.12 & 0.520 & 0.0364 & 0.49 & 1.40 & 19.19 & +0.5 & +0.11 \\
\hline $9 g F^{*}$ & 6200 & 3.76 & 544. & 0.20 & 0.611 & 0.0371 & 0.62 & 1.80 & 18.62 & +0.7 & +0.15 \\
\hline
\end{tabular}


TABLE 2 (cont.)

\begin{tabular}{|c|c|c|c|c|c|c|c|c|c|c|c|}
\hline $\begin{array}{l}\text { Number } \\
\text { and } \\
\text { Harmonic }\end{array}$ & $\begin{array}{c}\mathrm{T}_{\mathrm{e}} \\
\left({ }^{\mathrm{O}} \mathrm{K}\right)\end{array}$ & $\begin{array}{l}\text { Radius } \\
\left(10^{11} \mathrm{~cm}\right)\end{array}$ & $\begin{array}{c}\mathrm{g} \\
\left(\mathrm{cm} / \mathrm{sec}^{2}\right)\end{array}$ & $\begin{array}{l}\text { Convective } \\
\text { Fraction }\end{array}$ & $\begin{array}{l}\text { Period } \\
\text { (days) }\end{array}$ & $\frac{Q}{\left(P \sqrt{\rho / \rho_{\odot}}\right)}$ & $\begin{array}{l}\text { Ion Time } \\
\text { Period }(F)\end{array}$ & $R$ & $v_{0.83}$ & $\frac{\Delta E}{\%} / E$ & $\begin{array}{l}\text { Phase } \\
\text { Lag }\end{array}$ \\
\hline
\end{tabular}

Sequence $10 \quad 45 \% \mathrm{He}$

10a H $7700 \quad 2.44 \quad 1959$.

10b H $7400 \quad 2.64 \quad 1672$.

$\begin{array}{llll}10 \mathrm{cH} & 7100 \quad 2.87 & 1417 .\end{array}$

IOd H 6800

3.13

1192.

10e H 6500

3.42

995.

824.

676.

$\log \mathrm{H} \quad 5900$

IOh F

$$
5900 \quad 4.15
$$

676.

$L=1.50 \times 10^{35} \mathrm{erg} / \mathrm{sec}$

$M=1.75 \times 10^{33} \mathrm{gm}$

Sequence $11 \quad 45 \% \mathrm{He}$

$112 \mathrm{H} \quad 6800$

3.13

1802.

IIb H 6500

3.43

1505.

Sequence 12

12a $\mathrm{F} \quad 7100$

3.89

$12 \mathrm{~b}$

6800

4.24

$12 c$

6500

4.65

\section{$45 \$ \mathrm{He}$}

330 .

278.

232.

0.032

0.15

$L=1.50 \times 10^{35} \mathrm{erg} / \mathrm{sec}$

$$
0.178 \quad 0.0255
$$

0.202

0.0255

0.232

0.0259

0.265

0.0261

0.304

0.0262

0.355

0.0265

0.417

0.0268

0.23

0.29

0.36

0.45

0.56

0.72

0.99

0.99

0.44

0.557

0.0358

$M=2.65 \times 10^{33} \mathrm{gm}$

0.51

0.64

0.243

0.0257

$L=2.80 \times 10^{35} \mathrm{erg} / \mathrm{sec}$

0.024

0.0382

0.18

0.033

0.961

0.0392

0.22

0.051

0.0405

0.26
0.823

$M=0.75 \times 10^{33} \mathrm{gm}$

$\begin{array}{llll}0.74 & 17.27 & -2.8 & -0.068 \\ 0.94 & 16.52 & +3.1 & +0.016 \\ 1.26 & 15.73 & + & +0.077\end{array}$




\begin{tabular}{ccccccc}
$\begin{array}{c}\text { Number } \\
\text { and }\end{array}$ & $\mathrm{T}_{e}$ & Radius & $g$ & $\begin{array}{c}\text { Convective } \\
\text { Fraction }\end{array}$ & $\begin{array}{c}\text { Period } \\
\text { (days) }\end{array}$ & $\begin{array}{c}Q \\
\text { Harmonic } \sqrt{\rho / \rho_{\odot}}\end{array}$ \\
\hline
\end{tabular}

Sequence $13 \quad 45 \%$ He

$13 \mathrm{a} \mathrm{H} \quad 7400$

3.60

592.

$13 \mathrm{~b} \mathrm{H} * 7100$

3.91

$13 \mathrm{cH} * 6800$

4.26

13d $\mathrm{F} \quad 7100$

13 e $F * 6800$

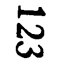

Sequence $14 \quad 45 \% \mathrm{He}$

14a H 7400

3.61

$14 \mathrm{~b} \mathrm{H} * \quad 7100$

3.92

$14 \mathrm{c} \mathrm{H} * 6800$

4.27

$14 \mathrm{~d} H * 6500$

4.67

14 e $\quad 6800$

4.27

$149 \mathrm{~F} * 6500$

4.67

$14 \mathrm{~g} F * 6200$

5.14

5.67
$L=2.80 \times 10^{35} \mathrm{erg} / \mathrm{sec}$

502.

422.

502.

422.

898.

762.

640 .

0.019

0.414

0.0268

0.029

0.476

0.0272

0.551

0.0276

0.638

0.0364

0.738

0.0370

$L=2.80 \times 10^{35} \mathrm{erg} / \mathrm{sec}$

0.029

0.327

0.0260

0.043

0.373

0.0262

0.067

0.429

0.0265

535.

0.11

0.498

0.0269

0.067

0.575

0.0355

0.668

0.0360

0.780

0.0365

0.920

0.0372
443.

363.
0.25
Ion Time Period (F)
$R \quad V_{0.83}$

$\frac{\Delta E}{\mathscr{6}} /$

Phase Lag

0.20

0.25

0.31

$M=1.0 .5 \times 10^{33} \mathrm{gm}$

0.18

$0.76 \quad 19.83$

$-0.8$

$0.97 \quad 19.34$

$+2.6$

$-0.095$

0.27

1.21

$18.80^{\circ}$

$+4.5$

$-0.075$

0.65

19.34

$-0.6$

$+0.021$

0.92

18.80

$+1.1$

$+0.030$

$M=1.75 \times 10^{33} \mathrm{gm}$

$0.52 \quad 20.65$

$-0.3$

$0.72 \quad 20.28$

$+0.8$

$-0.050$

$1.02 \quad 19.95$

$+1.3$

$-0.033$

0.40

1.40

19.50

$+1.5$

$+0.10$

0.31

0.76

19.95

$-0.0$

$+0.065$

0.40

1.07

19.50

0.51

1.45

19.07

0.66

18.49

$+0.050$

$+0.133$

$+0.148$ 


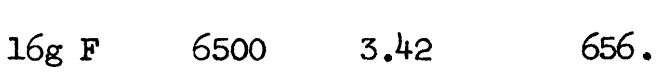

$0.521 \quad 0.0363$

0.69

$1.24 \quad 18.94+0.05+0.21$

TABLE 2 (cont.)

\begin{tabular}{|c|c|c|c|c|c|c|c|c|c|c|c|}
\hline $\begin{array}{c}\begin{array}{c}\text { Number } \\
\text { and } \\
\text { Harmonic }\end{array} \\
\end{array}$ & $\begin{array}{c}\mathrm{T}_{\mathrm{e}} \\
\left({ }_{\mathrm{K}}^{\mathrm{K}}\right) \\
\end{array}$ & $\begin{array}{l}\text { Radius } \\
\left(10^{11} \mathrm{~cm}\right)\end{array}$ & $\begin{array}{c}\mathrm{g} \\
\left(\mathrm{cm} / \mathrm{sec}^{2}\right) \\
\end{array}$ & $\begin{array}{l}\text { Convective } \\
\text { Fraction }\end{array}$ & $\begin{array}{l}\text { Period } \\
\text { (days) }\end{array}$ & $\begin{array}{c}Q \\
\left(P \sqrt{\rho / \rho_{\odot}}\right) \\
\end{array}$ & $\begin{array}{l}\text { Ion Time } \\
\text { Period (F) }\end{array}$ & $R$ & $v_{0.83}$ & $\frac{\Delta E}{\mathscr{g}} / E$ & $\begin{array}{l}\text { Phase } \\
\text { Lag }\end{array}$ \\
\hline & & Sequence 15 & $60 \% \mathrm{He}$ & \multicolumn{3}{|c|}{$L=1.50 \times 10^{35} \mathrm{erg} / \mathrm{sec}$} & \multicolumn{4}{|c|}{$M=0.75 \times 10^{33} \mathrm{gm}$} & \\
\hline $15 \mathrm{a} \mathrm{H}$ & 8000 & 2.26 & 982. & 0.014 & 0.252 & 0.0265 & 0.21 & 0.70 & 19.77 & -0.5 & -0.03 \\
\hline $15 \mathrm{~b} \mathrm{H}$ & 7700 & 2.43 & 845. & 0.019 & 0.286 & 0.0269 & 0.25 & 0.87 & 19.47 & +1.5 & +0.04 \\
\hline $15 \mathrm{~d} \mathrm{H}$ & 7100 & 2.86 & 611. & 0.043 & 0.373 & 0.0275 & 0.37 & 1.22 & 18.72 & +2.5 & +0.16 \\
\hline $15 \mathrm{e} F *$ & 7100 & 2.86 & 611. & 0.043 & 0.504 & 0.0371 & 0.37 & 1.09 & 18.72 & +0.9 & +0.069 \\
\hline $15 f \mathrm{~F}^{*}$ & 6800 & 3.12 & 514. & 0.063 & 0.583 & 0.0377 & 0.45 & 1.28 & 18.23 & +1.4 & +0.111 \\
\hline $15 \mathrm{gF}$ & 6500 & 3.41 & 430 & 0.097 & 0.682 & 0.0386 & 0.57 & 1.34 & 17.60 & +1.8 & +0.19 \\
\hline & & Sequence 16 & $60 \% \mathrm{He}$ & \multicolumn{3}{|c|}{$L=1.50 \times 10^{35} \mathrm{erg} / \mathrm{sec}$} & \multicolumn{3}{|c|}{$M=1.15 \times 10^{33} \mathrm{gm}$} & & \\
\hline $16 \mathrm{aH}$ & 8000 & 2.26 & 1504 . & 0.021 & 0.200 & 0.0259 & 0.25 & 0.53 & 20.62 & -0.4 & +0.026 \\
\hline $16 \mathrm{~b} \mathrm{H}$ & 7700 & 2.44 & 1291. & 0.029 & 0.223 & 0.0259 & 0.28 & 0.65 & 20.27 & +0.2 & +0.065 \\
\hline $16 \mathrm{c} \mathrm{H}$ & 7400 & 2.64 & 1101. & 0.041 & 0.255 & 0.0262 & 0.36 & 0.86 & 20.02 & +0.7 & +0.084 \\
\hline $16 \mathrm{dH}$ & 7100 & 2.87 & 934. & 0.059 & 0.291 & 0.0264 & 0.44 & 1.09 & 19.66 & +1.0 & +0.14 \\
\hline $16 \mathrm{e} \mathrm{H}$ & 6800 & 3.13 & 786. & 0.093 & 0.333 & 0.0266 & 0.55 & 1.38 & 19.33 & +0.8 & +0.21 \\
\hline $16 \mathrm{f} \mathrm{H}$ & 6500 & 3.42 & 656. & 0.14 & 0.387 & 0.0270 & 0.69 & 1.71 & 18.94 & +0.3 & +0.28 \\
\hline
\end{tabular}


TABLE 2 (cont.)

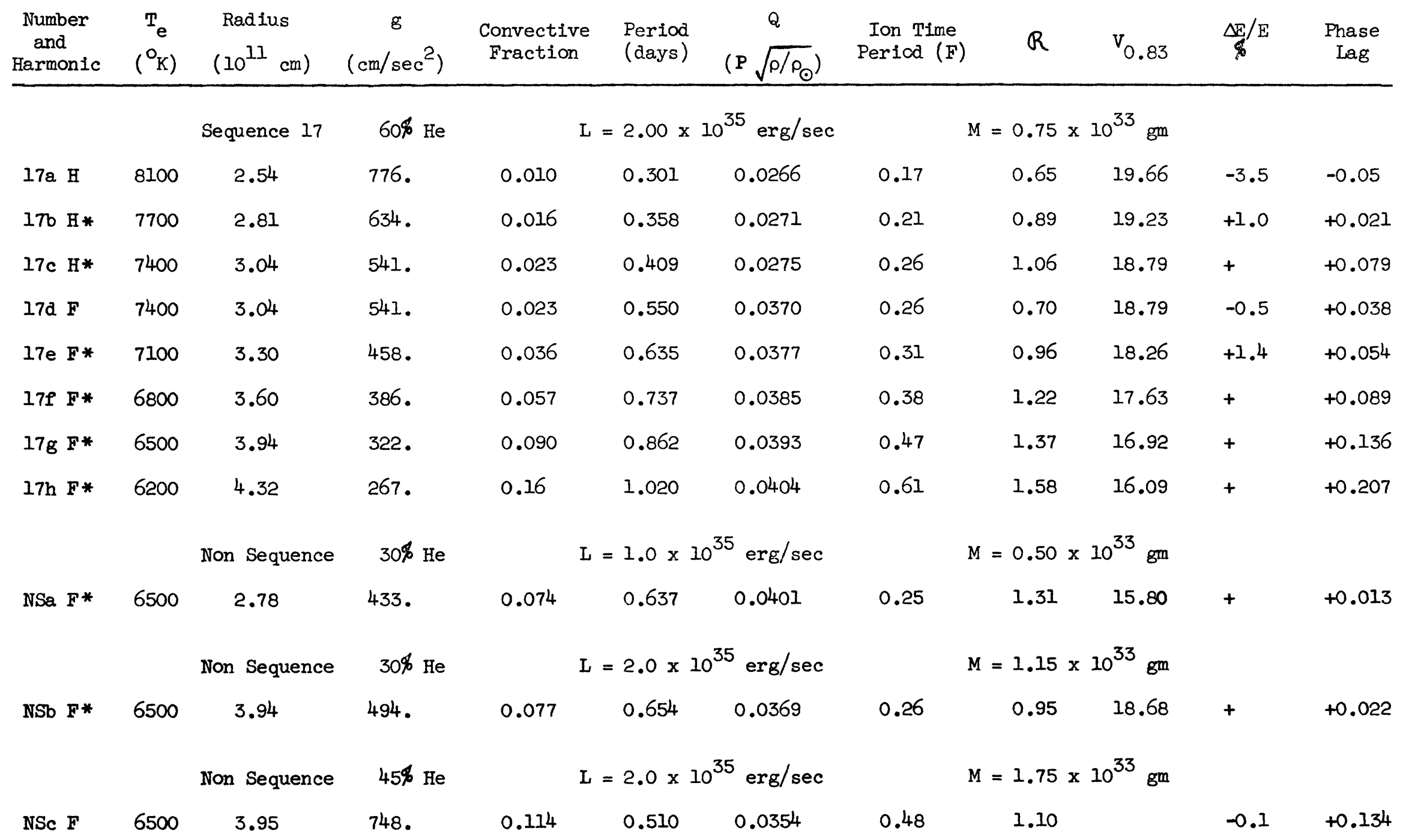


transition is much deeper than in the static case (Paper I, p. 895). This measure of the phase is closely related to the phase integral defined by Cox and Whitney (1958) but is particularly suited to a non-linear calculation. The eleventh column gives the stability result - the percentage increase $(+)$ or decrease $(-)$ in the kinetic energy of the envelope per period. The ninth, tenth, and twelfth columns are referred to later.

\section{c) The Types of Instability}

The normal sequence of results for fixed mass and varying $T_{e}$ is shown by sequence 5 of Table 2. As $T_{e}$ is decreased from a high value, in general the instability appears first in the first harmonic. The value of $T_{e}$ at which the instability first appears marks the blue side of the instability strip and can be found by interpolating the results for $\Delta E / E$. This limit also marks the shortest period of the sequence of unstable models with fixed $L_{0}$ and $M$.

As $T_{e}$ is decreased, there comes a minimum $T_{e}$ at which the first harmonic is unstable at small amplitude. However, before this $T_{e}$ is reached, there is another $T_{e}$ at which the fundamental first becomes unstable at small amplitude. There is thus a range of $T_{e}$ in which both modes are able to grow from small amplitude. This overlap of instability is primarily of theoretical interest.

What is of more interest observationally is that $T_{e}$ (the transition $T_{e}$ ) at which the final, stable, large-amplitude motion switches from the first harmonic to the fundamental. This question is, however, one of the most difficult to answer since it is just in this region of $T_{e}$ that both the fundamental and first harmonic motions are most persistent and, as a result, the calculation must be followed for a long time to determine which mode (if either) will ultimately dominate. Clearly, in this region are there four distinct possibilities at any given $T_{e}$. First (type 1), the first harmonic may ultimately settle down as a pure periodic motion regardless of initial conditions. Second (type 2), the fundamental may ultimately settle down as the pure periodic motion for arbitrary initial conditions. Third (type 3), the final motion may be either a pure fundamental or a pure first harmonic, depending on the initiating small-amplitude motion, that is, depending on the history of the star. Fourth (type 4), the final large-amplitude motion may be a permanent mixture of the fundamental and first harmonic. Since the ratio of the periods of these motions is very near 4 to 3 in the RR Lyrae region, such a mixture appears as a motion (fundamental) with a modulation period of about 3 periods or as a motion (first harmonic) with a modulation period of about 4 periods. Such motions are known among the $\delta$ Scuti variables. Since the period ratio is not precisely 4 to 3 , such motions are not strictly periodic. In this lies the major computational difficulty in establishing whether a harmonic mixture will persist: as the motion is essentially somewhat irregular, it is very difficult to define in a short time, whether the motion is persistent or not. In spite of the computational difficulty, the $\delta$ Scuti variables are undoubtedly undergoing this type of motion.

Of these four types of behavior, we have identified the first three only in these calculations. The fourth type may exist among these models, but, because of the inherent diffculty in its demonstration, it has not been proved. Typically, in the region of $T_{e}$ where both harmonics can grow at low amplitude, the first type (final first harmonic) is what is found at highest $T_{e}$. Next, as $T_{e}$ is lowered, there appears to be a region of type 3 motion where either the first harmonic or the fundamental can be found to be stable at large amplitude depending on initial conditions. At still lower $T_{e}$, the second type (stable fundamental) is found, and this continues even to lower $T_{e}$ where the first harmonic no longer shows a small amplitude instability.

Several examples (5d H, 5g F; 5e H, 5h F; 8c H, 8d F; 9e H, 9f F; 13c H, 13e F; $14 \mathrm{~d} H, 14 \mathrm{f} \mathrm{F}$ ) have been found for which the type 3 situation prevails. The ease with which this has been found points to the existence of a region of $T_{e}$ with this behavior. It is, however, quite difficult to establish the precise limits of this kind of motion: i.e., it is 
difficult to find the minimum and maximum $T_{e}$ for which either one or the other motion is stable depending on the initiating conditions. The reasons for this difficulty are clear as the question can be answered only by studying the motion at maximum amplitude and no interpolation method has been found to interpolate between the (necessarily) discrete examples explored.

The effect of such behavior in an evolutionary sequence would be as follows. If the star moves across the variable region toward lower $T_{e}$, the first-harmonic motion would persist to lower $T_{e}$ and longer periods before it switched to the fundamental. If, on the other hand, the star moves across the variable region toward higher $T_{e}$, the fundamental would persist to higher $T_{e}$ and shorter periods before switching to the first harmonic. In all the cases that have been examined, the decay of one harmonic or the other (as the case may be) has been so rapid that it would be essentially complete in about 100 periods. Even though some cases might take somewhat longer, it does not seem likely that cluster variables will be caught in the act.

As $T_{e}$ is lowered further, we presume the fundamental instability will diappear. However, in most of the models studied here, the method of calculation by which these motions has been studied became somewhat unreliable at essentially the same place where the star appeared to be stabilizing. For this reason, the lower limit of $T_{e}$ at which the instability finally ceases can only be estimated. This limit corresponds to the longest period in a series of fixed $L_{0}$ and mass.

For models with low mass for a particular luminosity (or high luminosity-to-mass ratio), the sequence of instabilities was quite different in that no region of first-harmonic instability was found. The high- $T_{e}$ boundary of the instability occurred where the fundamental first showed small-amplitude instability and the fundamental continued to the low $T_{e}$ region as the only unstable mode. As the mass was further decreased, there was no indication that the unstable region would disappear no matter how high the luminosity-to-mass ratio, although the region does move somewhat toward lower $T_{e}$.

On the other hand, for models with high mass for a particular luminosity, the firstharmonic instability which starts at the high $T_{e}$ boundary extends across the instability region to the low $T_{e}$ boundary and no region of fundamental instability is found. For still higher mass (smaller luminosity-to-mass ratio) the instability region remains a first-harmonic region but narrows and disappears entirely for $(L / L \odot) /(M / M \odot)$ less than about 30.

The above features of the instability region are shown in Figures 2-7. There, log $P$ (days) is plotted as ordinate against $-\log T_{e}$ (to give the same appearance as in the H-R diagram). The unstable models are plotted as $\odot$ 's and the stable ones as $X$ 's. The region of first-harmonic instability shows up separate from the region of fundamental instability because of the ratio of periods (which was nearly 1.34). The lines connecting the points are drawn to the limits of instability. In the region of overlapping fundamental and first-harmonic instability, the dotted vertical line shows approximately where the transition appears to take place between the two motions for large-amplitude pulsations.

It is possible that instability may exist in higher harmonics than the first under some circumstances. In this survey no systematic search for such higher harmonics has been made. With one exception, no indication of instability in a higher harmonic has appeared in any of these calculations (in that case, there was some indication of a thirdharmonic instability). Since the number of zones used in the models was relatively small $(\sim 38)$ in this survey, we would not expect to be able to get good results for high harmonics and this question has not been pursued.

\section{d) Systematics of the Instability Region}

From the results we have presented in Table 1 on the high- $T_{e}$ boundary of the instability region, it is possible to identify the particular features of the static-envelope models which apparently govern this limit of stability. An examination of Table 2 shows 
that the quantity (time to ionize surface layers)/(period of fundamental) is remarkably constant and about 0.2 at this limit; it is tabulated in the ninth column of Table 1 . This suggests that it is, in fact, just this physical limitation that the ionization regions must be able to delay the temperature rise by a significant fraction of a period and thereby be able to develop a significant amount of work to drive the pulsation-which controls the high- $T_{e}$ boundary of the instability.

Accordingly, we have systematized the data on this limit in the expression

$$
\left(T_{e}^{\max } / 10^{4}\right)^{5} \sqrt{ }\left(\frac{L / L \odot}{M / M \odot}\right)\left(X+X^{2}\right)=K \approx 1.9,
$$

where $X$ is the mass fraction of hydrogen. $K$ is tabulated in the tenth column of Table 1 ; its approximate constancy indicates it can be used to define the maximum $T_{e}$ for any model. This expression then serves to define $T_{e}{ }^{\max }$ for any model in the RR Lyrae region and can be extrapolated into the Cepheid region.

In order to allow for the metal content, we have explored the effect of metal content, $Z$, on static models only. If our surmise is correct that it is the heat capacity of the ionization regions that is significant, we can extend the above formula to include $Z$ by the results of these static envelopes. There follows

$$
K^{\prime}=\left(T_{e}^{\max } / 10^{4}\right)^{5} \sqrt{ }\left(\frac{L / L \odot}{M / M \odot}\right)\left(X+X^{2}\right)(1+6 Z) \approx 1.9
$$

in the range $Z=0-0.08$. This formula can now be used also in the Cepheid region for both population I and II Cepheids provided the ratio $L / M$ is known. This expression

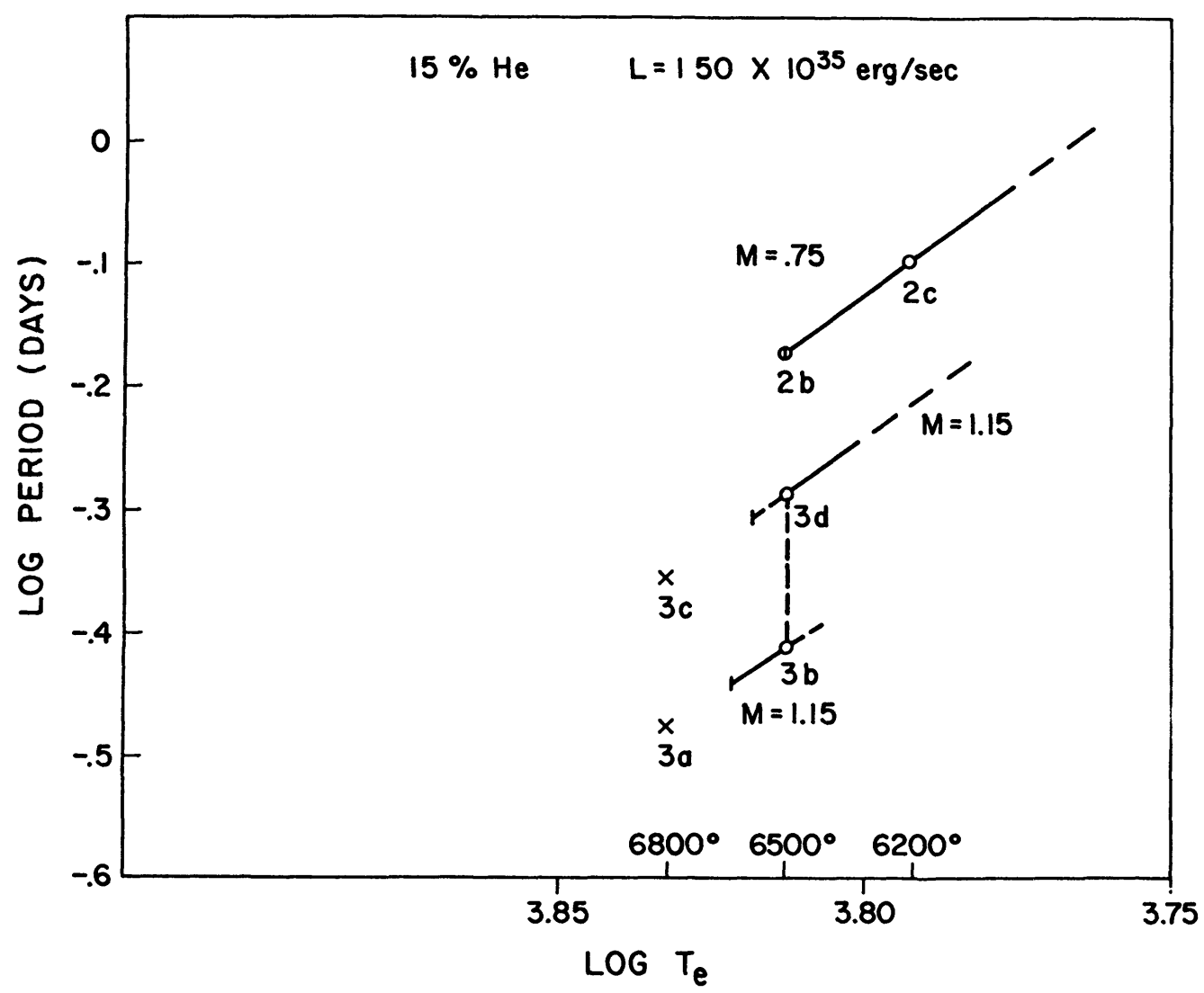

Fig. 2.-The dependence of the period on $T_{e}$ and the limits of instability for sequences 2 and 3 . The $X$ refers to a stable model, $\bigcirc$ to an unstable model, and the dashed vertical line marks the transition between fundamental and first harmonic. 
shows only about a 1 per cent decrease in $T_{e}{ }^{\max }$ for each 1 per cent increase in $Z$ and therefore suggests that metal content has an inappreciable effect on pulsation as such, although it may well have a profound effect on the stellar interior and thereby on $L$ and $M$.

We note in particular that the smaller the mass for a given $L$, the lower is $T_{e}{ }^{\max }$. This then means that a population II Cepheid (W Virginis type) will show instability at lower $T_{e}$ than classical Cepheids (as observed) if the masses of the population II Cepheids are considerably smaller. A separate publication will discuss a computed model of W Virginis with $M / M \odot=0.88$ that agrees well with observation and indeed is relatively cool.

In order to plot the above relation in the H-R diagram, a mass-luminosity relation is needed as well as a $(B-V)-T_{e}$ relation. We will not attempt to give such a plot here, but we note that we have found no significant disagreement between this relation and observation.

A noteworthy feature of the calculated models which is contained in this formula is the systematic shift of the instability region from low values of $T_{e}$ for low helium content to high values of $T_{e}$ for high helium content. If accurate values of the mean $T_{e}$ can be determined for pulsating stars, in particular for cluster variables, it is possible to use this dependence of the stability region on helium content to determine the helium content of actual variables or clusters. The calculated shift in the boundary is about a 0.5 per cent increase in $T_{e}$ for a 1 per cent increase in helium content. The application of this

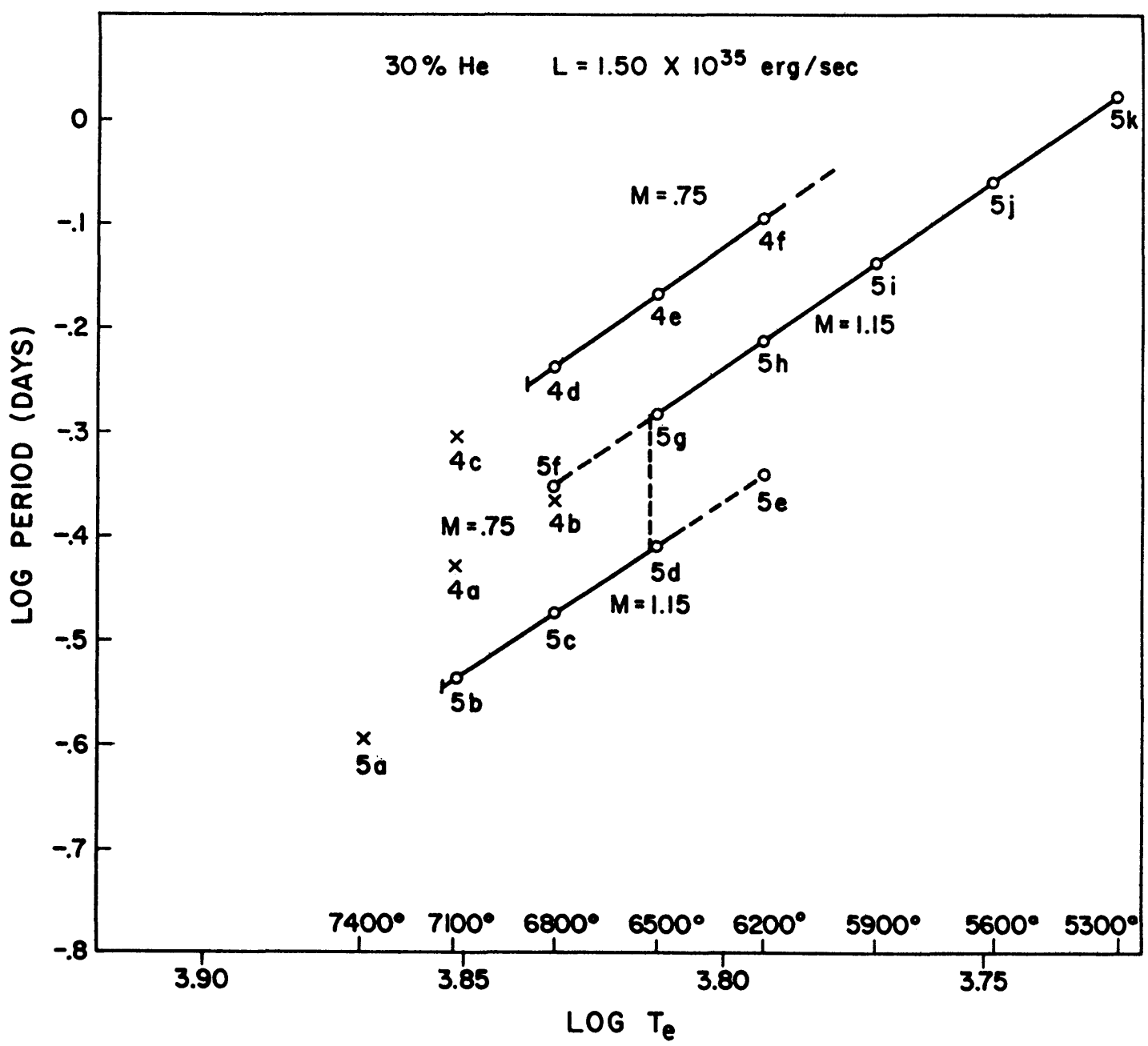

Fig. 3. - The dependence of the period on $T_{6}$ and the limits of instability for sequences 4 and 5 . The symbols are as in Fig. 2. 
relation to a cluster, where the limits of the instability region are directly observed, is immediate. The application to field variables and clusters is discussed in a later section.

We cannot so readily arrive at criteria for the low $-T_{e}$ limit of instability in view of the limitation of the calculations. We have, however, noted that when the convective fraction becomes 30-50 per cent, as shown in Table 2 , the models show signs of restabilizing. This has suggested that it is the effective transport of heat by convection that actually limits the low- $T_{e}$ bound of instability. We note that the convective fraction

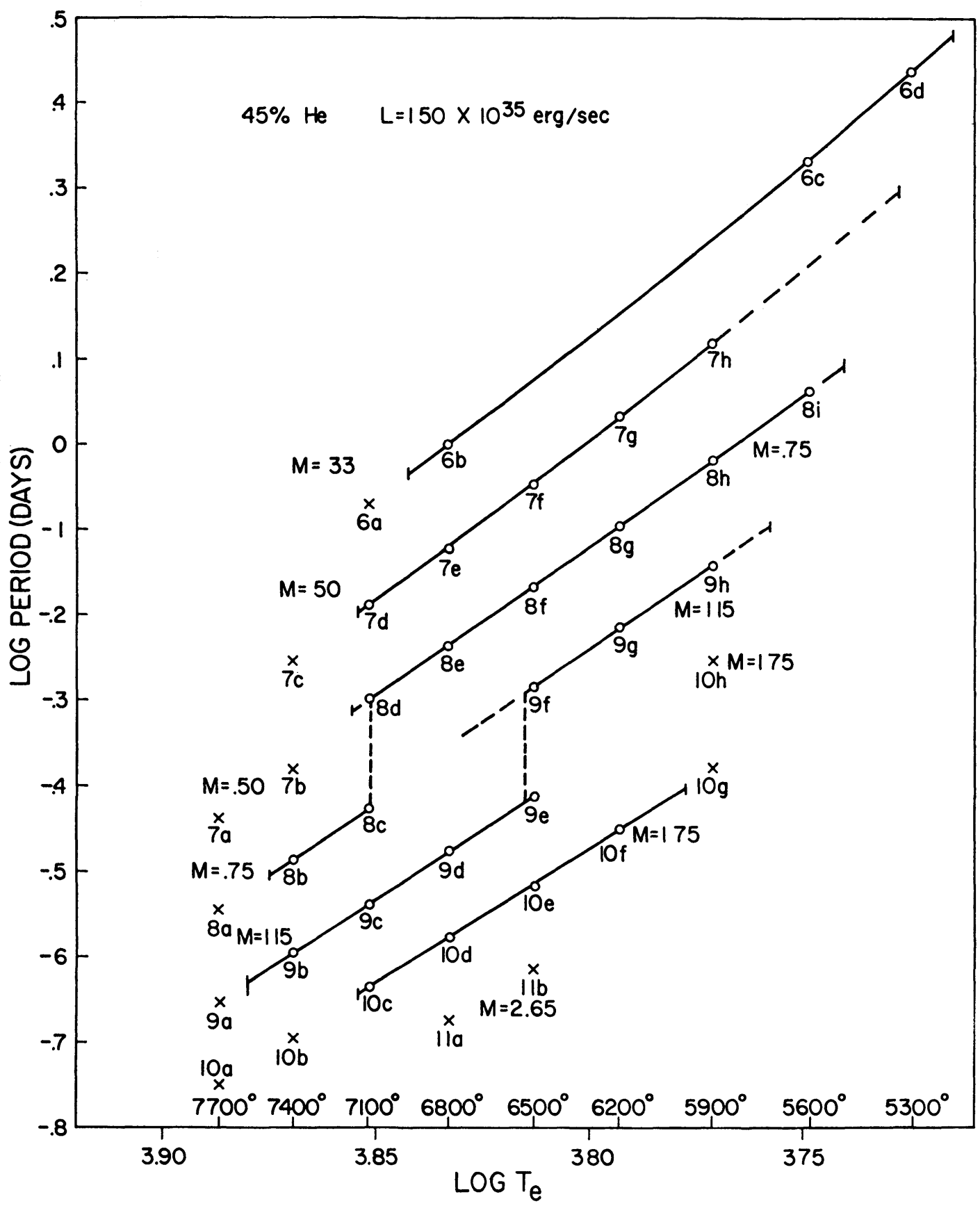

Fig. 4. - The dependence of the period on $T_{c}$ and the limits of instability for sequences $6-11$. The symbols are as in Fig. 2. 
actually is proportional to the square of the ionization time/period as shown in Table 2 so that (except for some dependence on $X / Y$ ) this value of the convective fraction also corresponds to a fixed value $\approx 1$ of ionization time/period. It has been shown (Cox 1963) that the ionization mechanism becomes less effective when the heat capacity of the ionizing layers becomes too great. Thus it appears that these two effects (increasing effectiveness of convection and decreasing effectiveness of ionization energizing) both take place at about the same place in the H-R diagram and both define lines parallel to the line given by equation (2) above but displaced in $T_{e}$. We estimate that the width of the unstable region corresponds to a ratio $T_{e}^{\max } / T_{e}^{\min } \approx 1.3$ to 1.4 . The fuller understanding of $T_{e}{ }^{\mathrm{min}}$ must await a study of the interaction of convection in the hydrogen zone with pulsation since the question of the persistence of pulsation to lower $T_{e}$ depends on the effectiveness of the hydrogen zone as an energizing influence in the presence of convection.

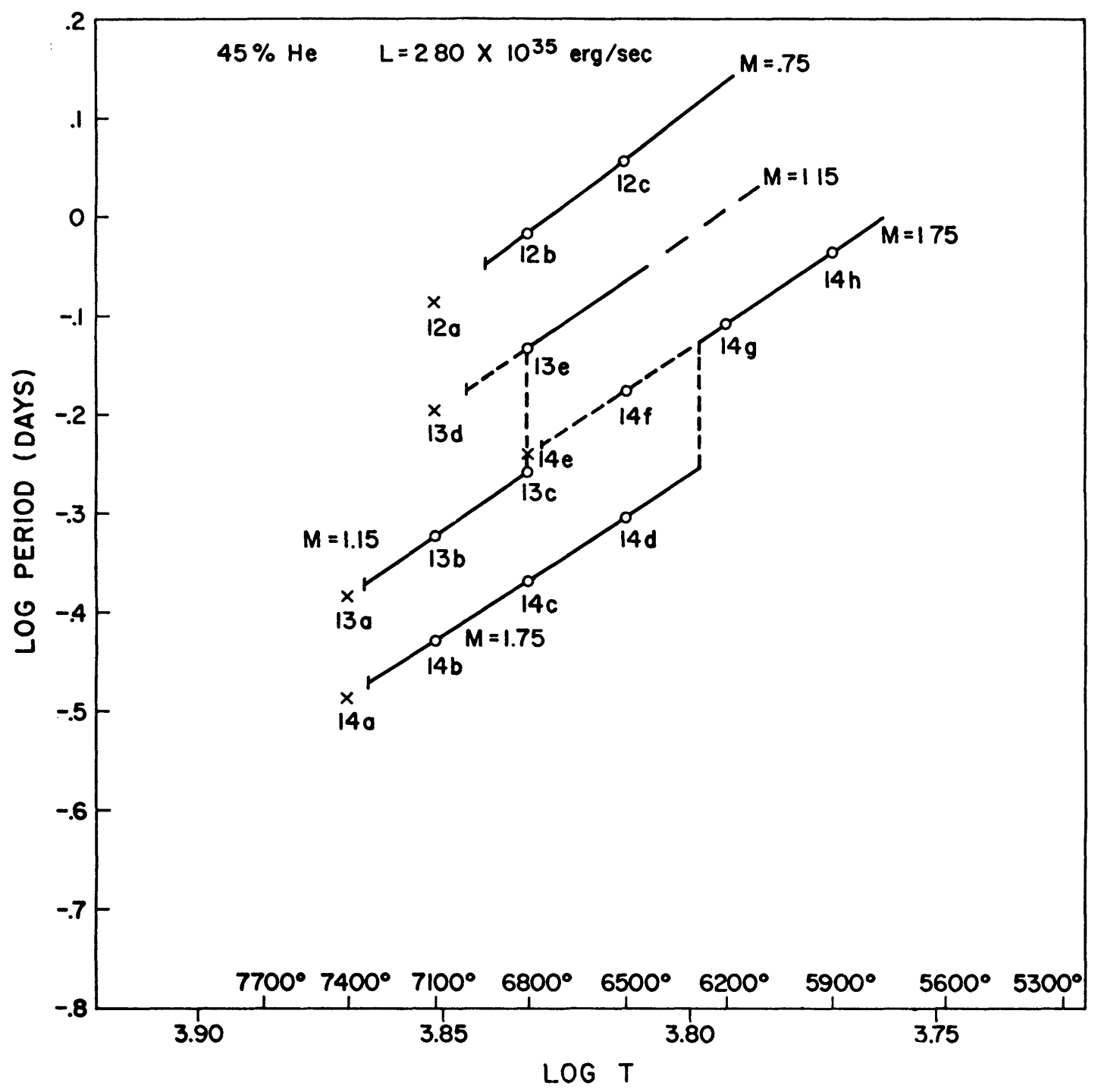

FIG. 5.-The dependence of the period on $T_{e}$ and the limits of instability for sequences 12-14. The symbols are as in Fig. 2. 


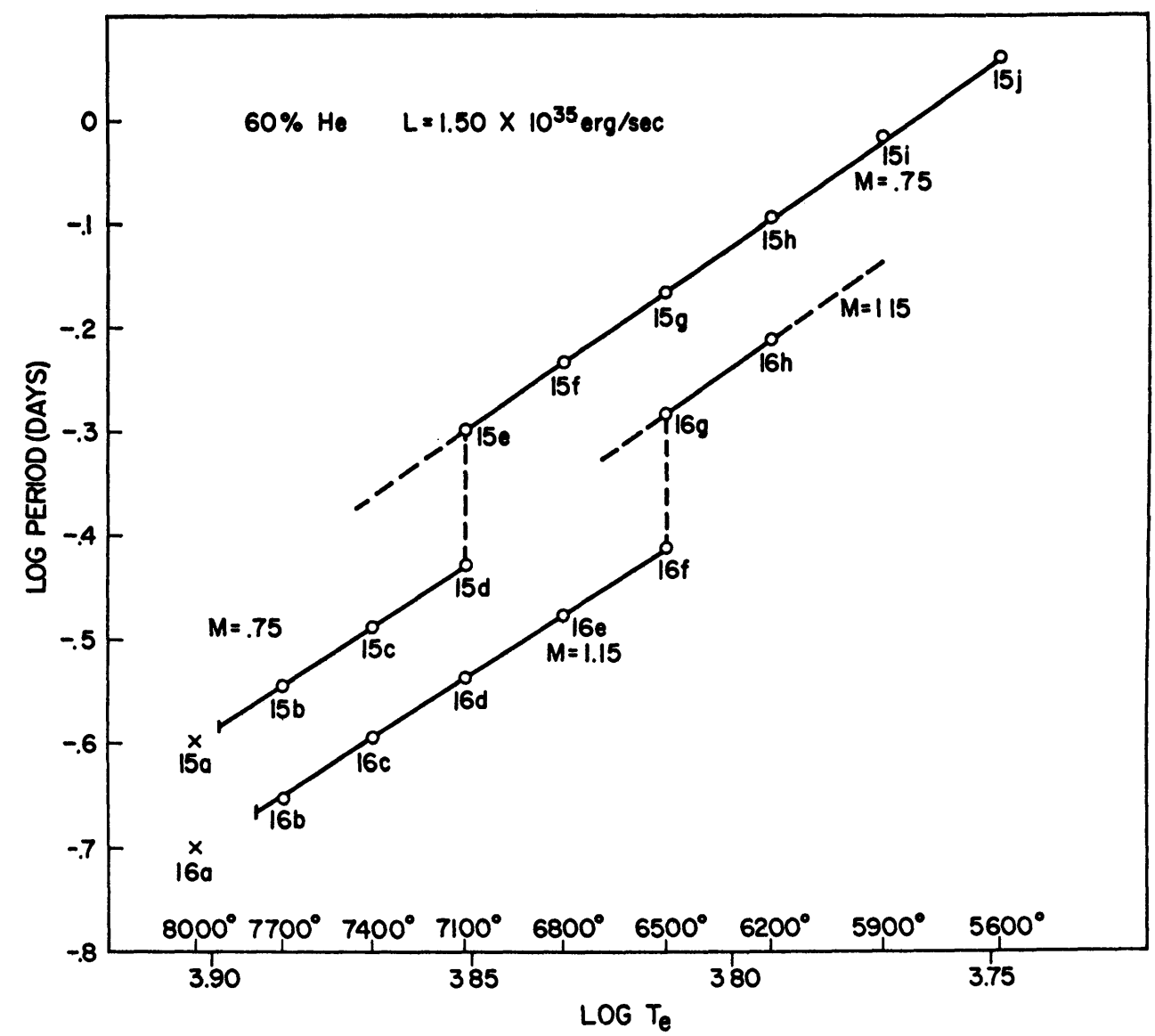

Fig. 6. - The dependence of the period on $T_{e}$ and the limits of instability for sequences 15 and 16. The symbols are as in Fig. 2.

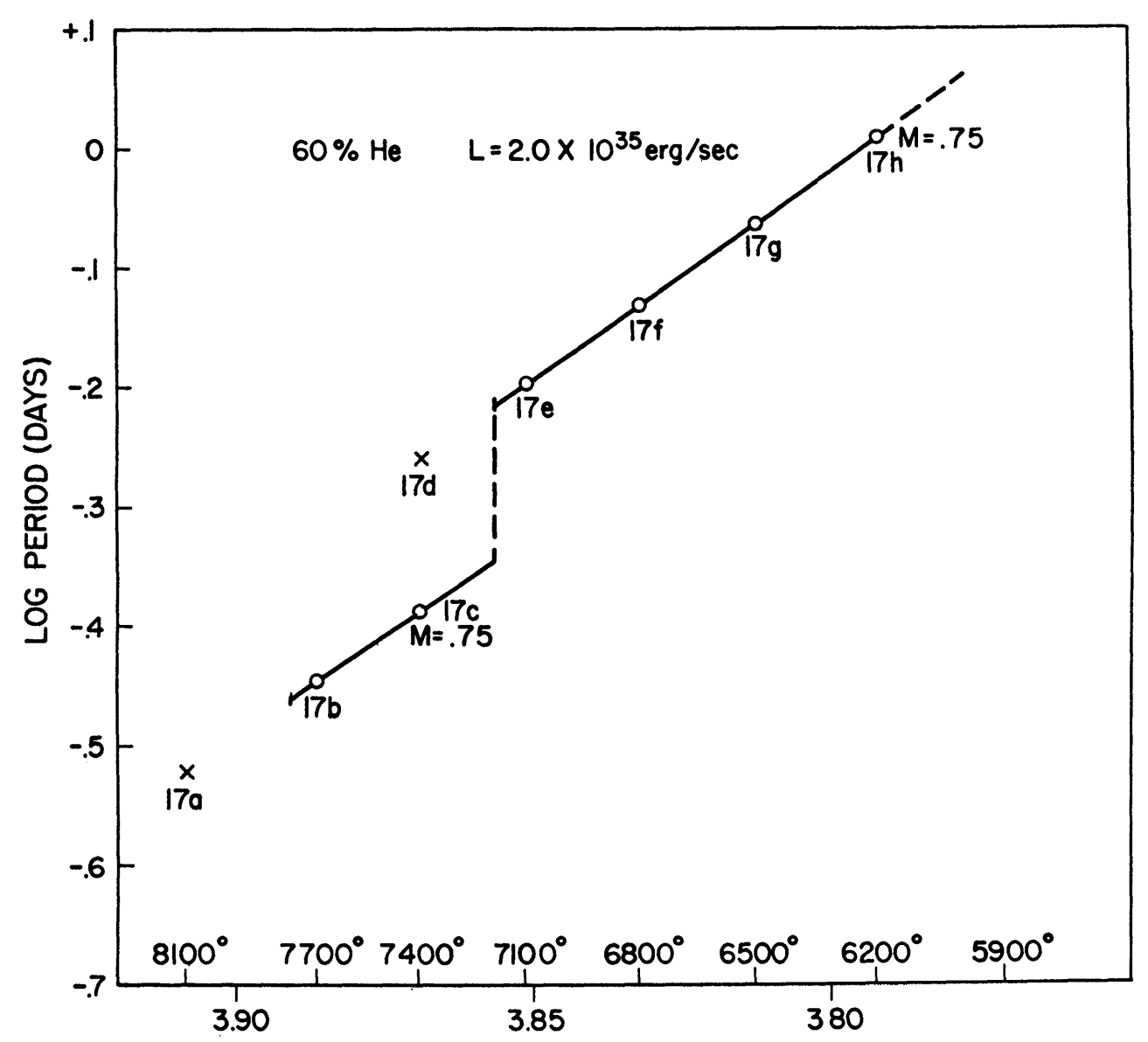

(C) American Astronomical Society - ProvidedG Je NASA Astrophysics Data System

FIG 7-The dependence of the period on $T$, and the limits of instability for sequence 17 The symbols 


\section{e) The Influence of Convection}

We have pointed out earlier that the calculations performed here have omitted convection as a means of energy transport. The method of Böhm-Vitense (1958) has been extensively used in model calculations but probably is unsatisfactory for models such as these (Mihalas 1965) as the computed velocities are near or exceeding sonic.

We have, however, tried to infer the nature of the changes that would be introduced by the presence of convection. Fundamentally, convection improves the efficiency of energy transport in the hydrogen zone. This is the same kind of effect as would be achieved by a reduction in opacity in this zone. We have seen that a reduction in opacity increases the mass of the ionizing regions (it increases the pressure at fixed temperature). And we have found that the instability occurs where the mass of the ionizing zones bears a certain ratio to the luminous flux per period. As a consequence, we see that the presence of convection will tend to shift the boundaries of instability somewhat to higher $T_{e}$. This shift will be quite small at the high- $T_{e}$ boundary we have computed as convection can have but little influence at higher $T_{e}$ because of the very low density of the envelope. However, it will have a much larger influence at the low- $T_{e}$ side where we have been unable to establish a clear boundary. It is likely that the shift will be large enough so that our lowest- $T_{e}$ models will actually lie outside the stable region.

Apart from such shifts in the instability boundary, especially at low $T_{e}$, we do not expect that the inclusion of convection will make major changes in the results. We note that changes in the opacity law would also have the effect of shifting the boundaries of instability. Presumably, the principal reason for the instability region moving toward higher $T_{e}$ with increasing helium content is just the decrease in opacity associated with the decrease in hydrogen content. Since we find that the parameter measuring convective efficiency is always very small at the high- $T_{e}$ boundary, it is suggested that these methods could be applied to any model to test for incipient pulsation instability without including convection. Such an investigation of $T_{e}^{\max }$ over a wide range of luminosities and masses is now under way.

\section{f) The Periods and a New Luminosity Relation}

A striking feature of Figures 2-7 is that the shortest-period fundamental (and, correspondingly, the longest-period first harmonic) is remarkably insensitive to mass. In other words, among all the periods plotted in Figure 4 there is a unique period that marks the shortest period of the fundamental where it changes to first-harmonic instability, independent of mass. In this figure it is very near 0.5 day.

We have explored this question in Figures 2-3 and 5-7 for compositions 15, 30, 45, and 60 per cent helium. The exploration is less thorough but the result is the same. The data on the transition from first harmonic to fundamental are summarized in Table 1. It appears that the transition temperature is the same for various compositions and fixed mass so that the transition appears at a fixed period independent of mass and composition.

However, the transition period $P_{\mathrm{tr}}$ (shortest fundamental period) does depend on the luminosity. The 0.5 -day value of $P_{\mathrm{tr}}$ was found for $L_{0}=1.50 \times 10^{35} \mathrm{ergs} / \mathrm{sec}$. From Figures 7 and 5 it appears that $P_{\mathrm{tr}} \approx 0.6$ day for $L_{0}=2.00 \times 10^{35} \mathrm{ergs} / \mathrm{sec}$ and $P_{\mathrm{tr}} \approx$ 0.75 day for $L_{0}=2.80 \times 10^{35} \mathrm{ergs} / \mathrm{sec}$. Actually, the range of luminosities explored here is not sufficient to define accurately the dependence of $P_{\operatorname{tr}}$ on $L_{0}$, but we have deduced the (approximate) relation

$$
P_{\mathrm{tr}} \approx 0.057(L / L \odot)^{0.6} \text { days }
$$

from the above results.

The significance of this result is immediately apparent. If we can observe a group of pulsating stars having uniform luminosity and showing both type $a$ and type $c$ (funda- 
mental and first-harmonic) pulsation, we can then identify the shortest-period type $a$ as $P_{\operatorname{tr}}$ and thereby deduce the absolute luminosity of the group. This method is applied in a later section to $\omega$ Cen, M3, and other clusters. We will discuss later the implications of this relation for Cepheid variables.

We have also attempted to find what properties of the static model govern this transition situation where the instability shifts from one harmonic to another. To this end, we have included in Table 2 the dimensionless quantity $V=-(r / p) d p / d r=\rho G M_{r} /(p r)$ evaluated at $r / R=0.83$. In this formula, $p$ is the pressure. This is the same quantity that is so useful in computations of stellar envelopes (Schwarzschild 1958, p. 109). We have chosen to evaluate $V$ at $r / R=0.83$, as this is where the node of the first harmonic comes and this point gives our best estimate of a representative or effective value of $V$ for the purposes of evaluating pulsation. We include the value of $Q_{F}$ and $V_{0}{ }_{83}$ at the transition in the last two columns of Table 1.

The transition conditions in the various sequences appear to correspond to a fixed value of $Q_{F} \approx 0.0365$ and of $V_{083} \approx 19.0$. This now permits us to examine any static envelope, and from the various criteria we have developed we can estimate whether it will be stable or unstable and whether the instability will favor the first harmonic or the fundamental.

We would also like to have been able to express the reasons for this result on the transition criterion in physical terms. So far, we have not found any convincing arguments that agree with the computed results.

The simplest representation of the period of a pulsating star is through the pulsation constant $Q=P_{(\text {days })} \sqrt{ }(\rho / \rho \odot)$ which is shown in the seventh column of Table 2 . It is apparent that $Q$ varies systematically in each sequence, increasing toward lower $T_{e}$. In order to include the first-harmonic cases, we have transformed the first harmonic $Q_{H}$ to a fundamental $Q_{F}$ by $Q_{F}=134 Q_{H}$.

It is found that $Q_{F}$ correlates very well with $V_{0}{ }_{83}$ except for sequence 1 . This correlation is shown in Figure 8. This is not surprising, as it is the run of $V$ with $r / R$ that determines $Q$ in the linear theory. This correlation permits the accurate estimation of the fundamental pulsation period for any calculated static envelope model without making any pulsation calculations.

No other general expression that would permit the determination of $Q$ for an arbitrary star has been found. In the present calculations, the series of results for $L \odot=1.50 \times$ $10^{35}$ can be digested into the form

which also implies

$$
Q_{F} \approx 0.033\left(\frac{9000}{T_{e}}\right)^{n}
$$

where, empirically,

$$
P \propto R^{15+n / 2}
$$

$$
n=0.18 M \odot / M .
$$

It must be noted, however, that this expression is valid only for the particular value of $L_{0}$ and cannot be extended to other luminosities.

\section{PROPERTIES OF UNSTABLE MODELS}

a) Phase Shifts, Amplitude Ratios, and Asymmetry of Luminosity-and Velocity-Curves

Several possible observable measures of phase relations, amplitude relations, and asymmetry have been studied in this survey so that quantitative comparisons of the calculated features of pulsation with observation could be made. These measures have all been found to vary systematically from one side of the instability region to the other so that it was hoped that they could be used to fix the location of a given star in the instability region and to extract additional physical properties of the star. 
The first of these quantities is the phase shift. We have attempted to define a phase shift that is readily found in the calculations but, more important, that is readily observable and insensitive to small inaccuracies of observation or peculiarities of a given variable. We chose to define the phase of the light-curve not by the time of peak light but rather by the time of mean light in the rising branch. Since the rise from minimum to maximum light occurs usually in much less than $\frac{1}{2}$ period, the time at which the luminosity $L$ equals its time-average value $L_{0}$ is quite precisely determined. Similarly, we have defined a fiducial phase of the velocity-curve as the time at which the velocity passes through zero (relative to the star) at minimum radius. The velocity referred to in

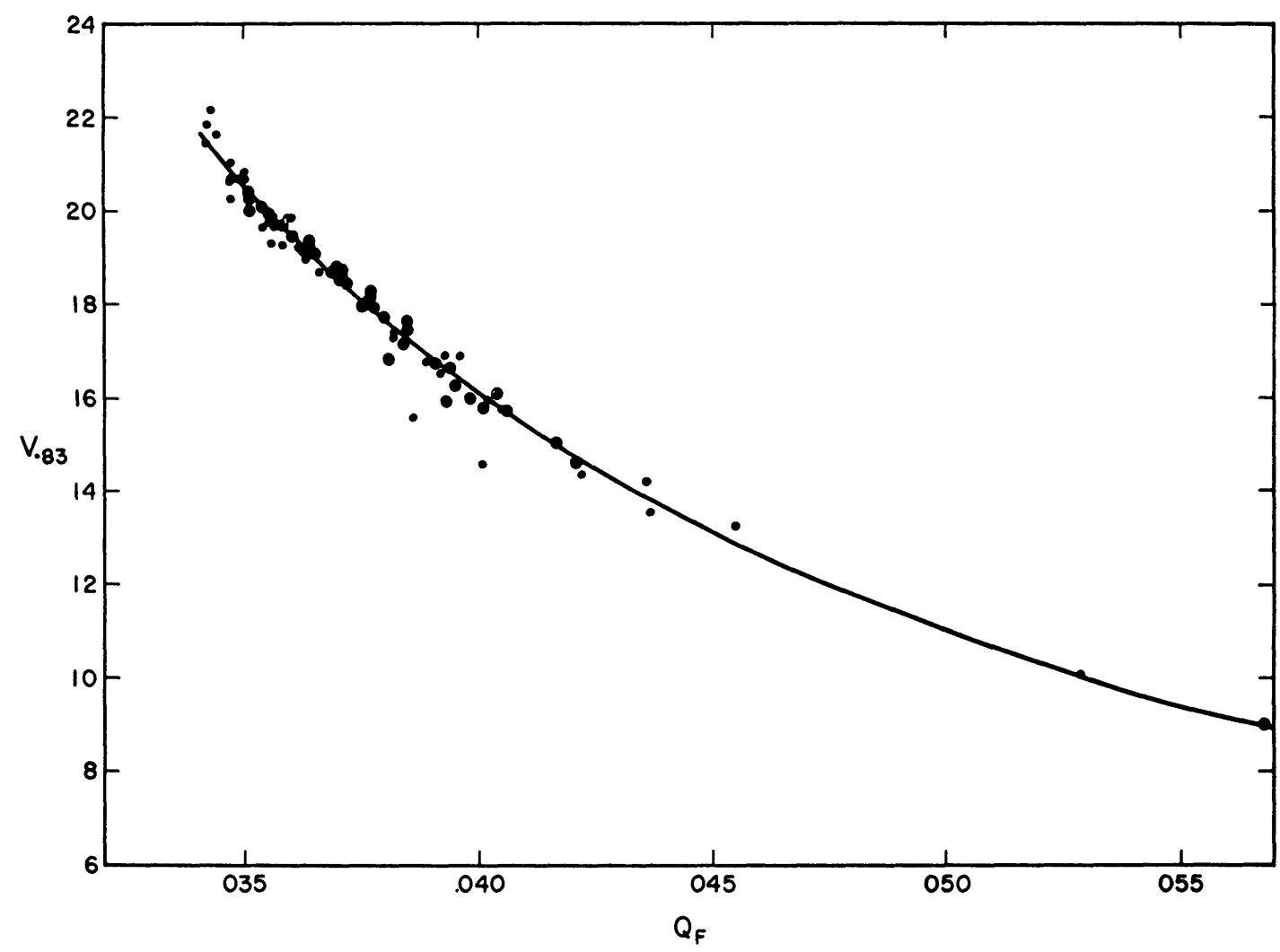

FIG 8 -The correlation of $V_{083}=d(\log$ pressure $) / d\left(\log\right.$ radius), evaluated at $r / R=083$, with $Q_{F}$ the pulsation constant of the fundamental mode. The large dots are the more precise models of Table 3 .

the calculation is the velocity of a fixed-mass layer located at about $\tau=0.1-0.2$ in the static model. It is known, of course, that the velocity observed depends somewhat on the spectral lines used and refers to varying mass layers in the atmosphere. The observed velocity tends to refer to a very deep layer at velocity maximum (astronomical convention on sign: infalling with respect to star) since it corresponds approximately to temperature minimum which is the opacity minimum, whereas the observation relates to a very shallow (in mass) layer at velocity minimum because of the (then), near maximum temperature. This leads in many cases to an observational doubling of the velocity (two lines being observed with low and high velocities) just at the time when the velocity is rapidly reversing. However, an examination of observed velocity-curves indicates that this doubling usually does not significantly confuse the time of zero velocity being referred to here. We will discuss later the question of a velocity gradient in the atmosphere and its relation to these calculations. We have finally defined the phase lag as the lag in time of the fiducial phase of the luminosity-curve after the corresponding fiducial 
phase of the velocity-curve divided by the period. With this definition, the characteristic " $\pi / 2$ " phase lag corresponds to zero lag as defined here. If the luminosity increase is abnormally late, we have a positive phase lag, whereas, if it is abnormally early, we have a negative phase lag.

The phase lag defined in this way was calculated for all the models studied. It was found (see Paper II) that, although the lag was relatively independent of amplitude, there was a small but systematic decrease in the lag between models at $\frac{1}{2}$ maximum and maximum amplitude. The lag is tabulated in the twelfth column of Table 2 as a fraction of the period. Because of the decrease with increasing amplitude, the lag is most accurate

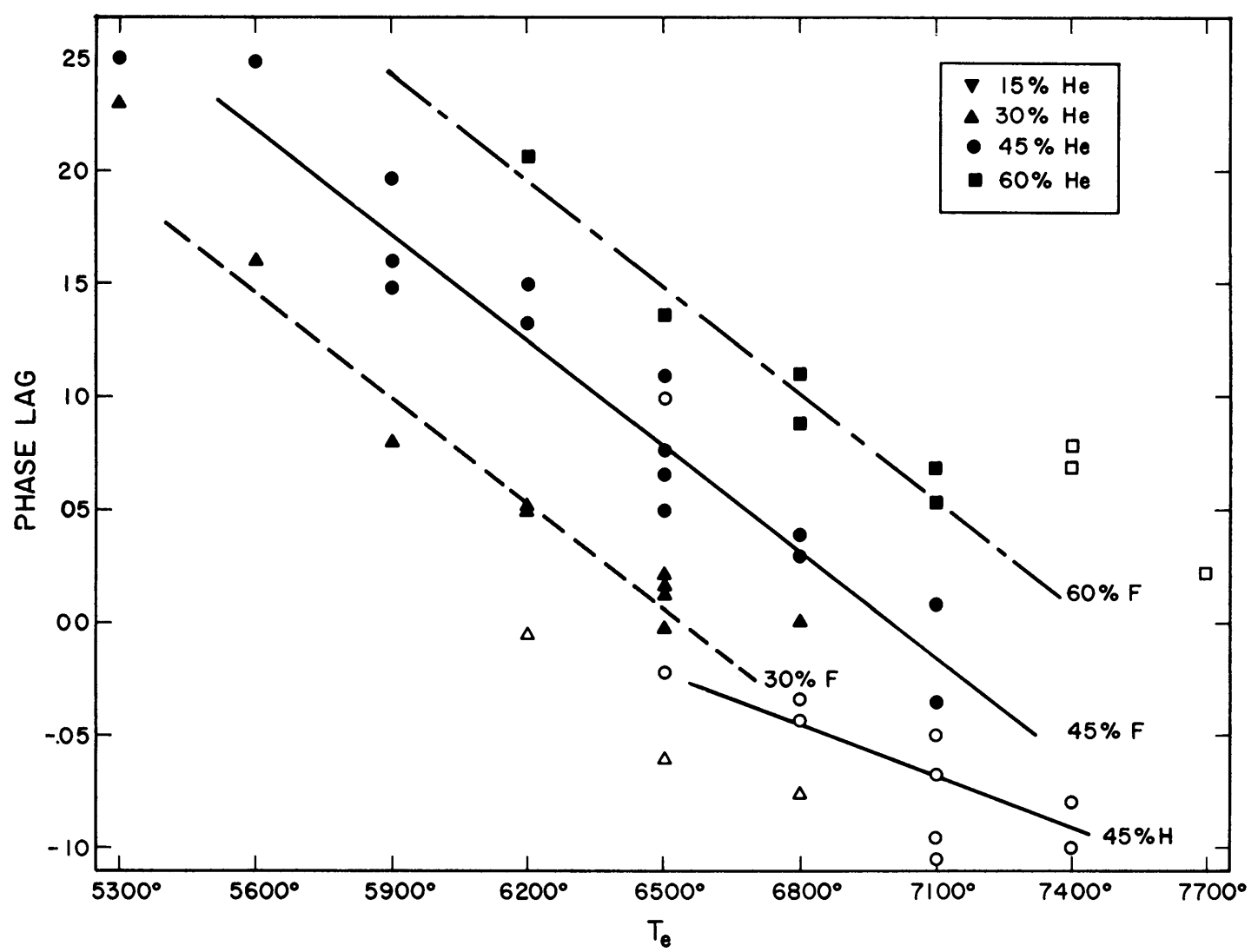

Fig. 9.-The correlation of the phase lag with $T_{e}$ for the models of Table 3. The solid symbols are fundamental modes; the open symbols are first-harmonic modes

for the starred models which were calculated at full amplitude and are listed also in Table 3. The general behavior of the phase lag as $T_{e}$ is varied across the instability region is as follows. The lag is usually negative for first-harmonic models with less than 60 per cent helium content. When the motion changes to the fundamental, the lag is small and usually positive. As $T_{e}$ decreases, the lag increases somewhat. This behavior is shown in Figure 9 where the phase lag for maximum-amplitude models only are plotted against $T_{e}$. The general behavior is the same for 30 and 45 per cent helium and (for the fundamental only) also for 60 per cent helium. The lag for the fundamental increases by about 0.10 for a $650^{\circ}$ decrease in $T_{e}$. The value of $T_{e}$ that corresponds to a fixed phase lag is lowest for 30 per cent and highest for 60 per cent helium. Thus for a lag of $0.05, T_{e}=$ $6220^{\circ}$ for 30 per cent $\mathrm{He}, T_{e}=6680^{\circ}$ for 45 per cent $\mathrm{He}$, and $T_{e}=7130^{\circ}$ for 60 per cent $\mathrm{He}$. The precise determination of the mean $T_{e}$ of pulsating stars may thus provide a measure of the helium abundance in their envelopes. 
TABLE 3. The Full Amplitude Survey

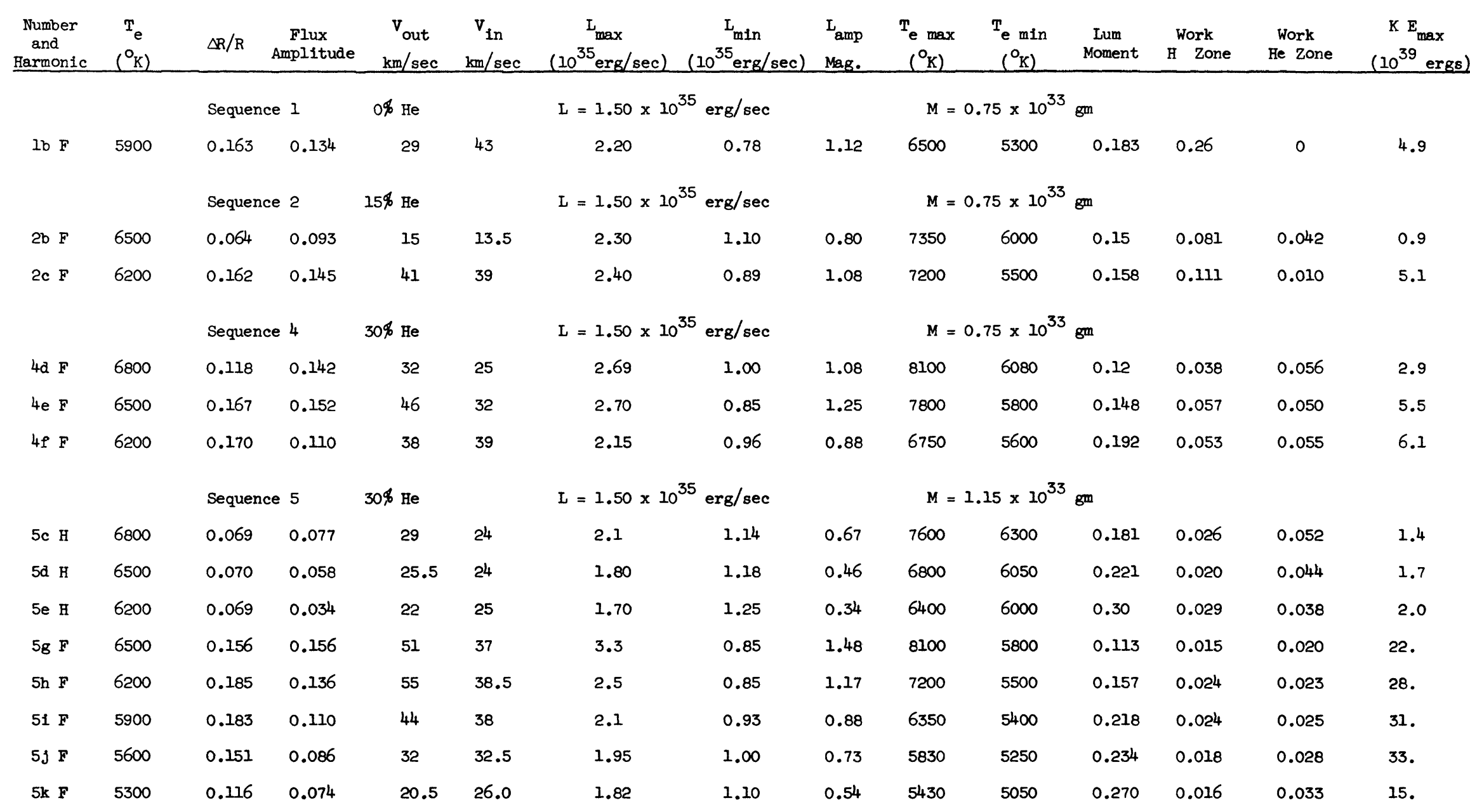


TABLE 3 (cont.)

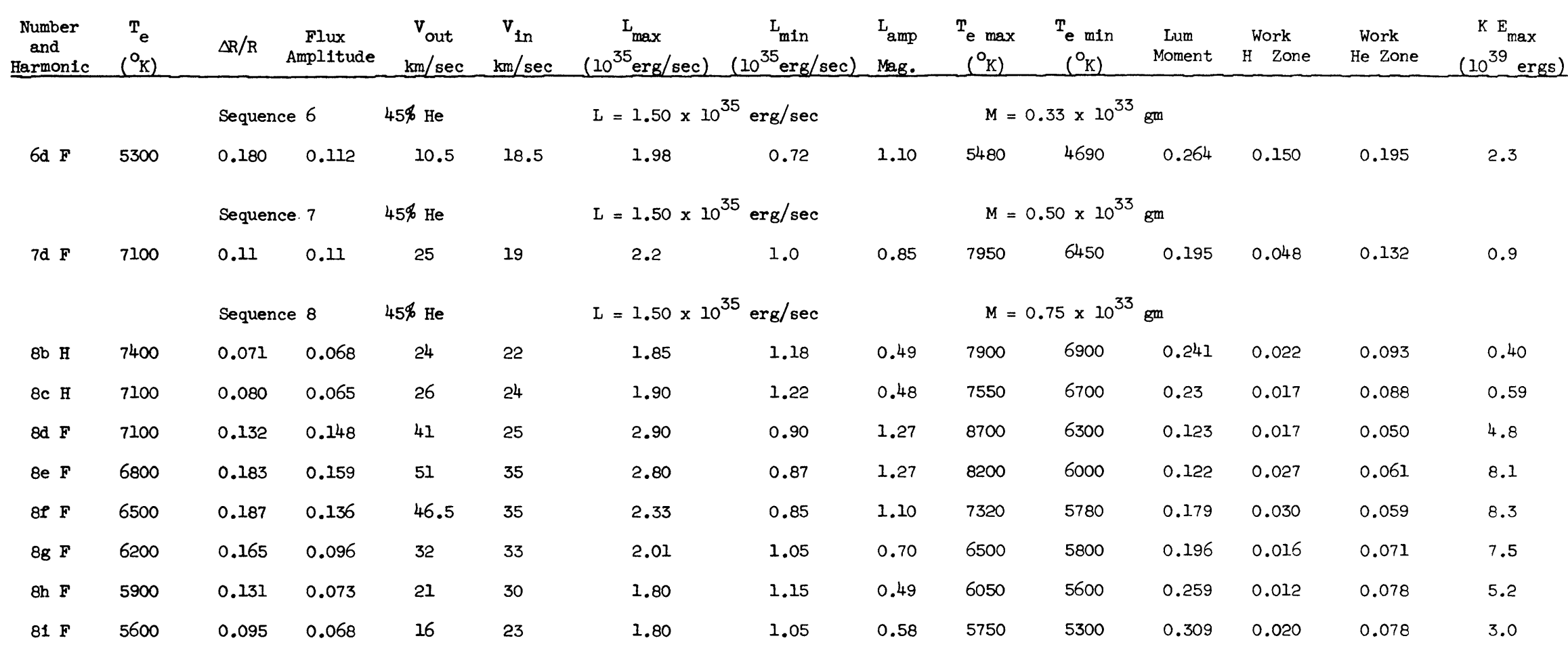


TABLE 3 (cont.)

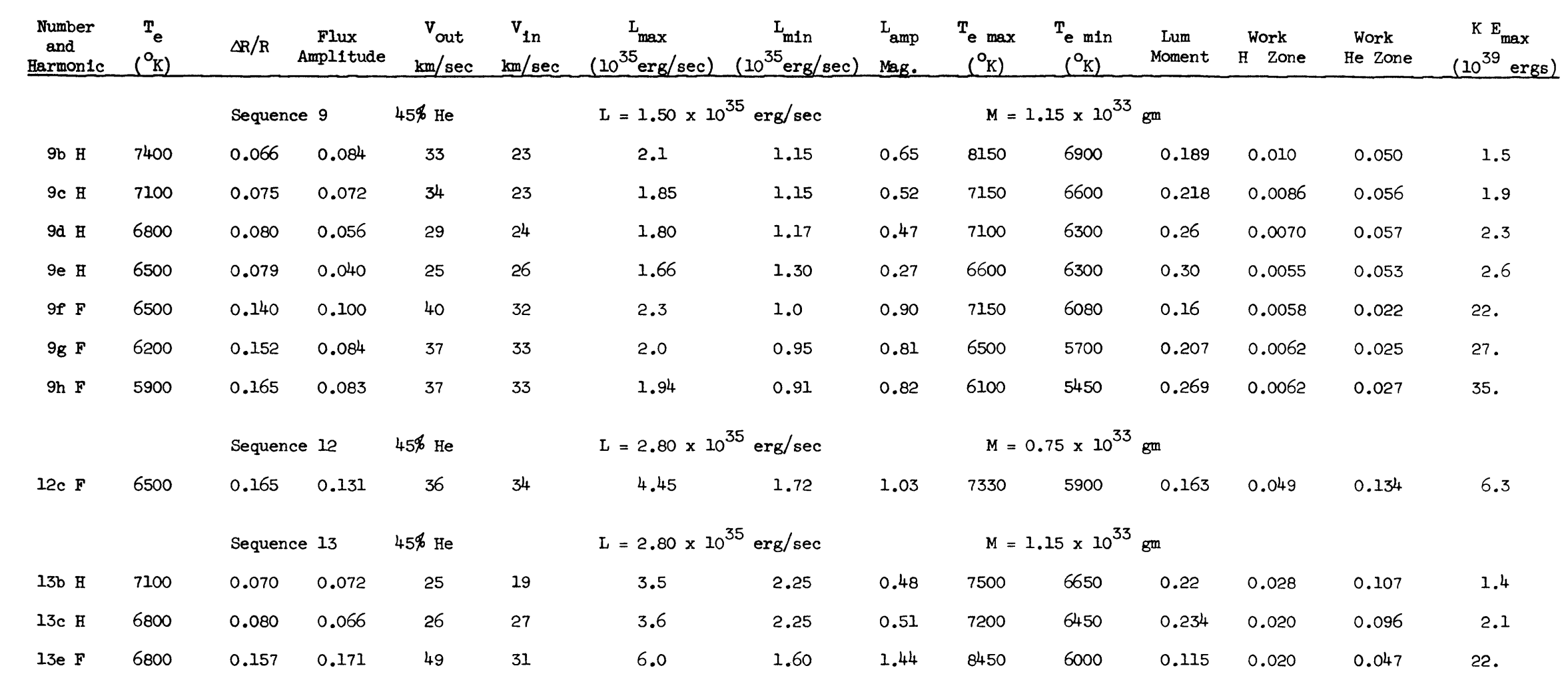


TABLE 3 (cont.)

\begin{tabular}{|c|c|c|c|c|c|c|c|c|c|c|c|c|c|c|}
\hline $\begin{array}{l}\text { Number } \\
\text { and } \\
\text { Harmonic }\end{array}$ & $\begin{array}{c}\mathrm{T}_{\mathrm{e}} \\
\left(\mathrm{O}_{\mathrm{K}}\right)\end{array}$ & $\Delta R / R$ & $\begin{array}{c}\text { Flux } \\
\text { Amplitude }\end{array}$ & $\begin{array}{c}\nabla_{\text {out }} \\
\mathrm{km} / \mathrm{sec}\end{array}$ & $\begin{array}{c}v_{\text {in }} \\
\mathrm{km} / \mathrm{sec}\end{array}$ & $\begin{array}{c}\mathrm{I}_{\max } \\
\left(10^{35} \mathrm{erg} / \mathrm{sec}\right)\end{array}$ & $\begin{array}{c}\mathrm{L}_{\min } \\
\left(10^{35} \mathrm{erg} / \mathrm{sec}\right)\end{array}$ & $\begin{array}{l}\mathrm{I}_{\text {amp }} \\
\text { Mag. }\end{array}$ & $\begin{array}{l}T_{e} \max \\
\left({ }^{\circ} \mathrm{K}\right)\end{array}$ & $\begin{array}{l}T_{e} \min \\
\left({ }^{\circ} \mathrm{K}\right)\end{array}$ & $\begin{array}{c}\text { Lum } \\
\text { Moment }\end{array}$ & \begin{tabular}{cc}
\multicolumn{2}{c}{ Work } \\
$\mathrm{H} \quad$ Zone
\end{tabular} & $\begin{array}{l}\text { Work } \\
\text { He Zone }\end{array}$ & $\begin{array}{c}K E_{\max } \\
\left(10^{39} \text { ergs }\right)\end{array}$ \\
\hline
\end{tabular}

\begin{tabular}{cccccc} 
菖 15 c H & 7400 & 0.080 & 0.065 & 29 & 19 \\
15 e F & 7100 & 0.166 & 0.152 & 53.5 & 33 \\
151 F & 6800 & 0.180 & 0.141 & 47 & 35 \\
& & \multicolumn{5}{c}{ Sequence 17} & $60 \%$ He \\
$17 \mathrm{~b} \mathrm{H}$ & 7700 & 0.078 & 0.088 & 28 & 23 \\
$17 \mathrm{c} \mathrm{H}$ & 7400 & 0.088 & 0.083 & 31 & 24 \\
17 e F & 7100 & 0.167 & 0.174 & 49 & 30 \\
$17 \mathrm{f} \mathrm{F}$ & 6800 & 0.177 & 0.145 & 46 & 34 \\
$17 \mathrm{~g} \mathrm{~F}$ & 6500 & 0.168 & 0.123 & 34 & 29 \\
$17 \mathrm{~h} \mathrm{~F}$ & 6200 & 0.129 & 0.082 & 21 & 28
\end{tabular}

$\begin{array}{cc}\mathrm{L}= & 2.80 \times 10^{35} \mathrm{erg} / \mathrm{sec} \\ 4.50 & 2.00 \\ 3.80 & 2.05 \\ 3.50 & 2.22 \\ 5.05 & 1.70 \\ 3.80 & 1.90 \\ 3.60 & 1.70\end{array}$

$\begin{array}{ccc} & M=1.75 \times 10^{33} \mathrm{gm} \\ 0.88 & 8100 & 6550\end{array}$

\begin{tabular}{|c|c|c|c|c|c|}
\hline $14 \mathrm{~b} \mathrm{H}$ & 7100 & 0.074 & 0.103 & 38 & 27 \\
\hline $14 \mathrm{c} \mathrm{B}$ & 6800 & 0.084 & 0.082 & 37 & 29 \\
\hline $14 \mathrm{~d}$ H & 6500 & 0.088 & 0.063 & 32.5 & 28 \\
\hline $14 f \mathrm{~F}$ & 6500 & 0.138 & 0.129 & 51 & 42 \\
\hline $14 \mathrm{~g} \mathrm{~F}$ & 6200 & 0.133 & 0.092 & 36 & 33 \\
\hline $14 \mathrm{~h} \mathrm{~F}$ & 5900 & 0.148 & 0.089 & 36 & 34 \\
\hline
\end{tabular}

$$
L=1.50 \times 10^{35} \mathrm{erg} / \mathrm{sec}
$$

$\begin{array}{llll}0.88 & 8100 & 6550 & 0.162\end{array}$

$0.67 \quad 7350$

6300

$1.18 \quad 7580$

6130

0.199

0.0111

$0.75 \quad 6600$

5900

0.23

0.0119

0.0423

6.1

$0.81 \quad 6100$

5800

0.145

0.0116

0.0493

8.0

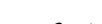

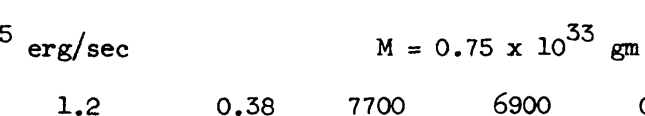

$\begin{array}{lllllllll}1.7 & 1.2 & 0.38 & 7700 & 6900 & 0.205 & 0.012 & 0.112 & 0.76 \\ 2.7 & 0.78 & 1.35 & 8400 & 6250 & 0.134 & 0.010 & 0.044 & 9.1 \\ 2.42 & 0.80 & 1.20 & 7600 & 6100 & 0.160 & 0.014 & 0.054 & 10.5\end{array}$

$L=2.00 \times 10^{35} \mathrm{erg} / \mathrm{sec}$

$\begin{array}{lllllllll}2.75 & 1.54 & 0.63 & 8400 & 7150 & 0.182 & 0.024 & 0.122 & 0.63 \\ 2.80 & 1.55 & 0.64 & 8050 & 6900 & 0.161 & 0.014 & 0.146 & 0.89 \\ 4.00 & 1.00 & 1.50 & 8500 & 6250 & 0.127 & 0.019 & 0.067 & 9.5 \\ 3.30 & 1.05 & 1.24 & 7700 & 6000 & 0.159 & 0.026 & 0.075 & 9.8 \\ 2.88 & 1.30 & 0.86 & 6950 & 6000 & 0.190 & 0.016 & 0.080 & 9.4 \\ 2.50 & 1.30 & 0.71 & 6400 & 5850 & 0.220 & 0.007 & 0.092 & 6.3\end{array}$


TABLE 3 (cont.)

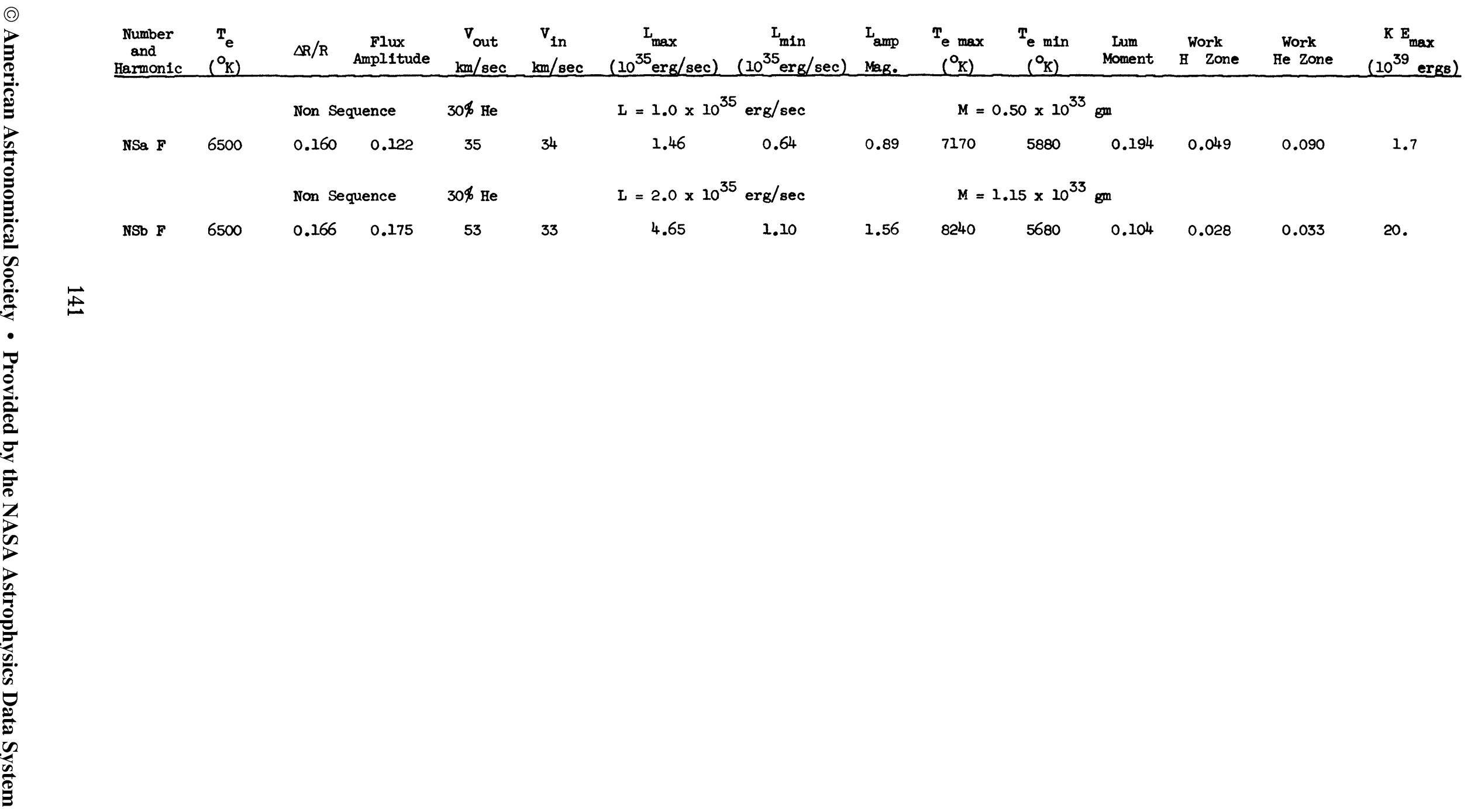


For the first-harmonic models for 30 and 45 per cent helium, the luminosity rise is earlier by about 0.07 at corresponding $T_{e}$ than for the fundamental. This is not the case for 60 per cent He. The reason is seen in reference to the corresponding calculated lightcurves. The 30 and 45 per cent He models show a prominent double peak in the luminosity whereas those for 60 per cent He do not. The first peak in the light-curve is responsible for the early phase for the lower helium models. This feature is discussed and compared to observation in a later section.

The predictions (Christy 1963a) about the increase in phase lag with decrease in $T_{e}$ that result from this survey have not yet been confirmed from observation If they are found to be true, they could afford a way of locating a field variable in the unstable region and thereby relating it to all of the results of this survey. Recently, Preston and Paczyński (1964) have observed the negative phase lag associated with the first-harmonic (type $c$ ) variables.

Studies have also been made to discover other observable measures of pulsating stars. One such measure could be taken to be the ratio of the velocity amplitude to the light amplitude, which is known to be relatively constant. However, peak amplitudes are particularly difficult to calculate accurately since any harmonic mixture or lack of precision will affect the amplitude. Observationally, peak amplitudes are also subject to significant errors. We have chosen instead to calculate the ratio $R$ defined as the ratio of the relative radius amplitude $\Delta R / R$ to the integrated luminosity amplitude (ILA)

$$
\text { ILA }=\frac{\int^{\text {positive phase }}\left(L-L_{0}\right) d t}{\text { period } \times L_{0}}
$$

$\Delta R$ is the full (not half) radius amplitude $\left(R_{\max }-R_{\min }\right)$. This ratio $R=(\Delta R / R) /$ ILA (see Paper II) is quite insensitive to amplitude and could be fairly accurately determined for medium as well as maximum-amplitude models. It is tabulated in the ninth column of Table 2. For reference, if there were no variation of $T_{e}$ during the period and if the radius (and luminosity) varied sinusoidally, $R$ would equal $\pi$. In general, the existence of a variation in $T_{e}$ in phase with the variation of $R$ reduces the value of $R$, and the larger the temperature amplitude, the smaller is $R$.

The ratio $R$ is shown plotted against $T_{e}$ in Figure 10 for $L=1.50 \times 10^{35}$. In addition, $R$ varies somewhat with $L$. In general, we see that $R$ decreases as $T_{e}$ increases and, for the first-harmonic models, $R$ is somewhat larger than for the fundamental corresponding to the smaller temperature amplitude of these models. Also, as for the phase lag, $\mathbb{R}$ tends to take on similar values at higher $T_{e}$ for higher helium content.

A measure of the asymmetry of the light-curve has also been explored in an attempt to find additional distinct observable measures. We have chosen (largely for reasons of precision and convenience) the luminosity moment defined as

$$
\text { Luminosity moment }=\frac{\int_{t_{0}}^{t_{1}}\left(t-t_{0}\right)\left(L-L_{0}\right) d t}{\operatorname{Period} \times \int_{t_{0}}^{t_{1}}\left(L-L_{0}\right) d t},
$$

where $t_{0}$ is the time when $L=L_{0}$ and is increasing, while $t_{1}$ is the time when $L=L_{0}$ and is decreasing. The luminosity moment is just the time moment of the positive phase of the luminosity-curve divided by the period. It is tabulated only for the full amplitude models in the twelfth column of Table 3 since it varies significantly with amplitude It would be 0.25 for a sinusoidal light-curve and 0.17 for a sawtooth light-curve.

The dependence of the luminosity moment on $T_{e}$ is shown in Figure 11. The wellknown trend to greater asymmetry at higher $T_{e}$ (Roberts and Sandage 1955) for funda- 


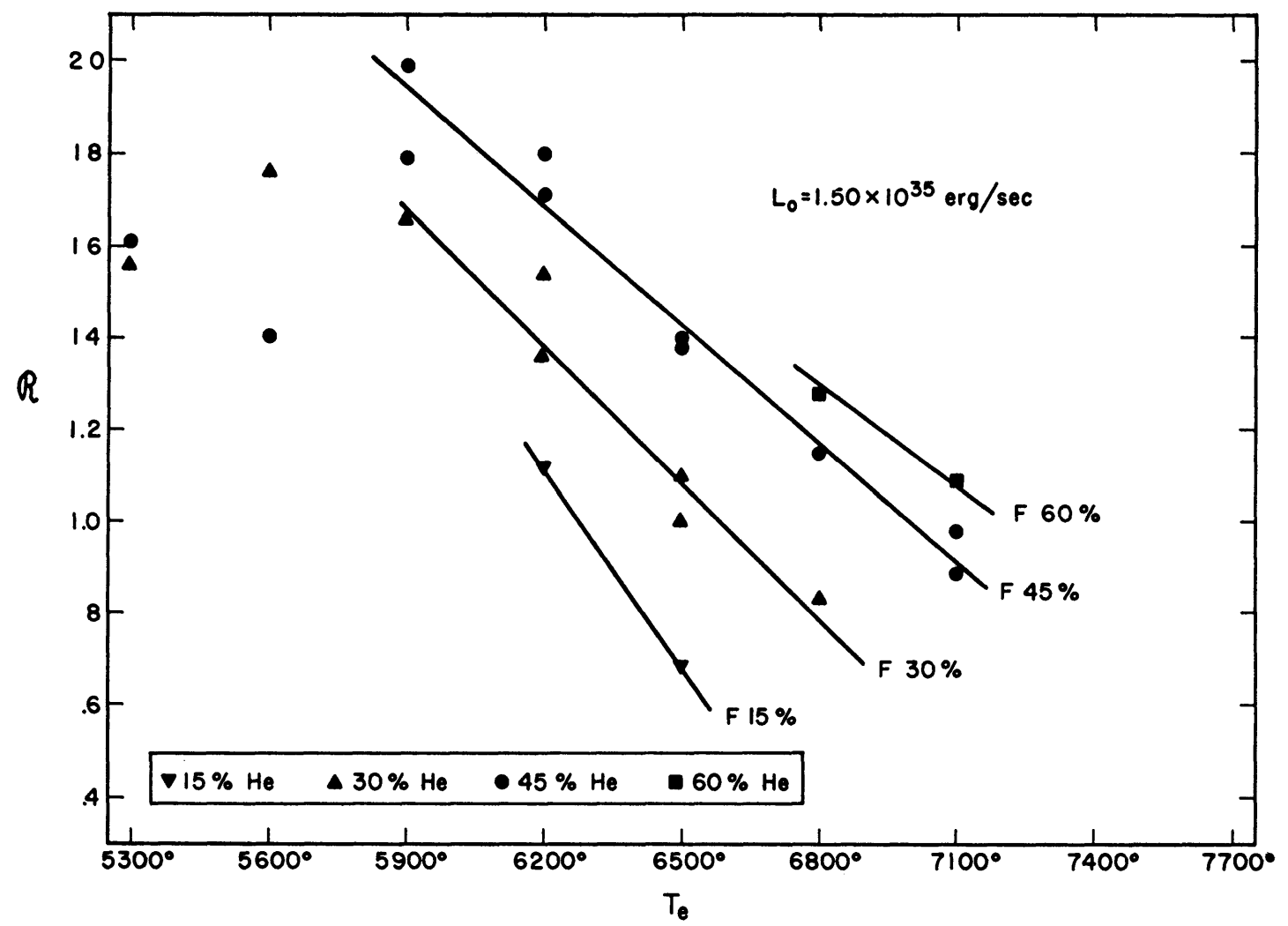

Fig. 10.-The correlation of the ratio $R$, of the radius amplitude to the integrated luminosity amplitude, with $T_{e}$ for the fundamental models of Table 3.

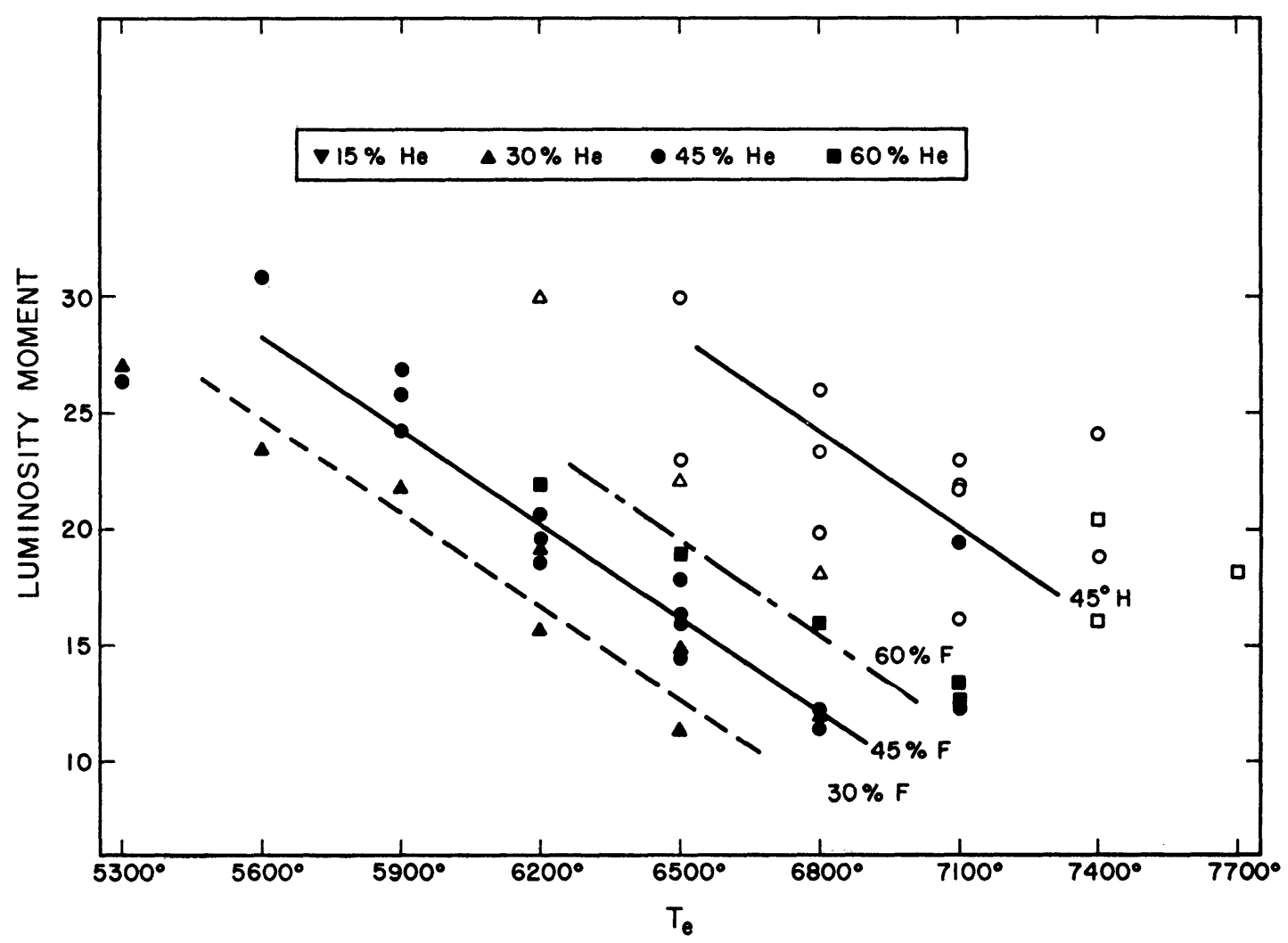

Fig. 11. - The correlation of the luminosity moment with $T_{e}$ for the models of Table 3. Symbols are a in Fig. 9. 
mental-mode pulsators, which is responsible for the type $b$ to $a$ variation, is apparent in the figure as a decrease in luminosity moment with increasing $T_{e}$. The results for different helium content show that the same behavior is found at $T_{e}$ increased by about $250^{\circ}$ for an increase of 15 per cent in helium content. The first-harmonic models show larger moments and less asymmetry which is the characteristic of type $c$ variables.

We have found, however, that the luminosity moment correlates closely with $R$. In Figure 12 we see that the very asymmetric light-curve, corresponding to a small luminosity moment, is found with small $\&$ corresponding to a large temperature amplitude. The

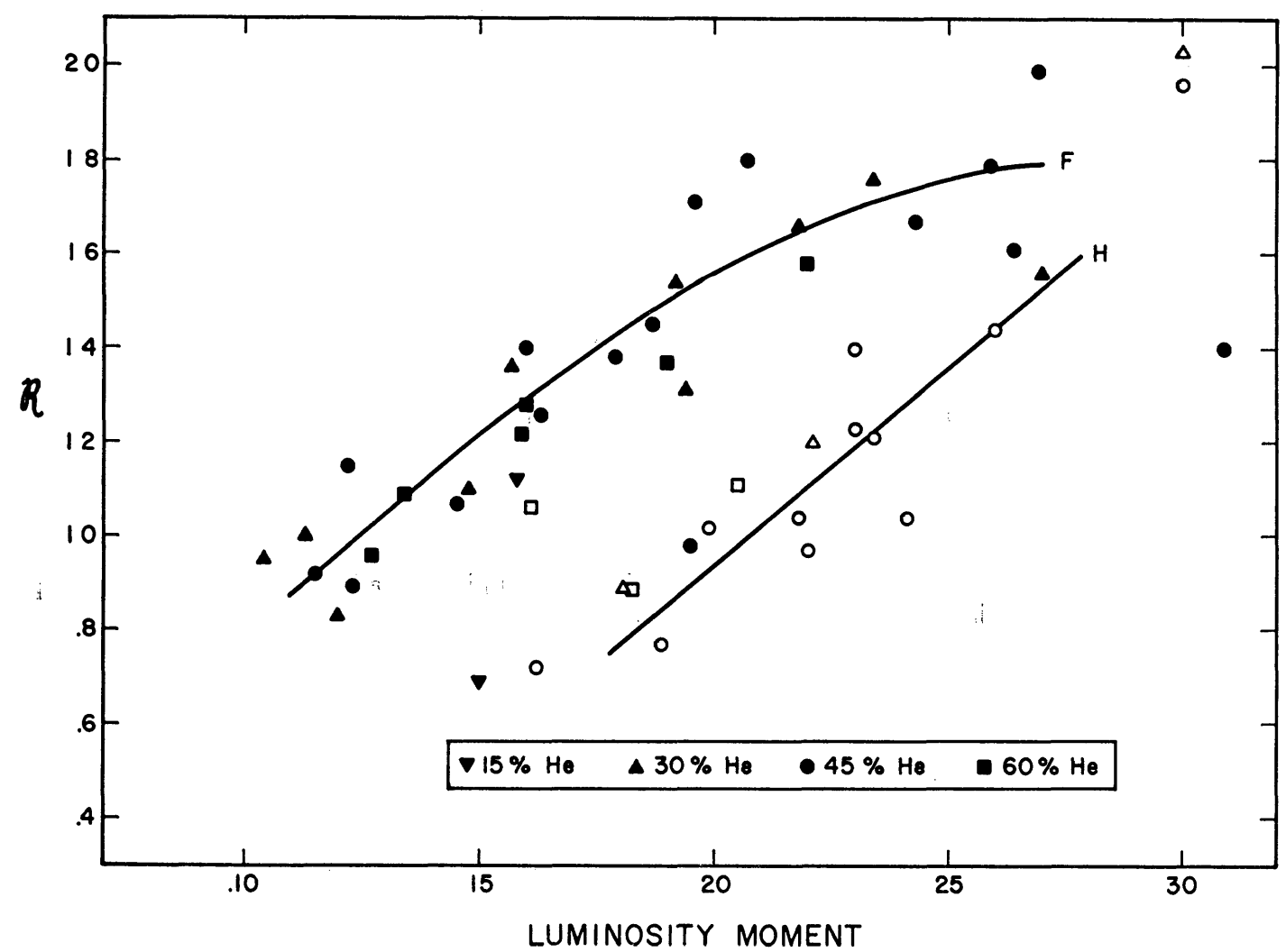

FIG. 12.-The correlation of the ratio $\mathcal{R}$, of the radius amplitude to the integrated luminosity amplitude, with the luminosity moment for the models of Table 3. Symbols are as in Fig. 9.

separate determination of $R$ and the luminosity moment would, however, clearly distinguish the first harmonic from the fundamental mode. Apart from this, the two measures contain basically the same information, and it is a matter of observational convenience and precision which measure is used.

The luminosity moment also correlates well with the phase lag (Fig. 13). Again, the first-harmonic mode is clearly distinguished from the fundamental. In the fundamental mode, some distinction of helium content is discernible with the higher helium content involving a larger phase lag for a given asymmetry. In Figure 14, $\mathbb{R}$ and the phase lag are compared with a similar result. For example, with a value of $R$ near 1 , there is a clear separation of the first harmonic at phase lag $\approx-0.07$, from the fundamental, and the phase lag, about 0.01 at 30 per cent helium in the fundamental, increases to about 0.06 at 60 per cent helium. This result offers a possibility of determining helium content and already has suggested that 30 per cent helium is nearer to correct than 60 per cent helium. Attempts to match these and other measures to an example (SU Draconis) have already been made (Christy 1963b) and are discussed fully in a later section. 


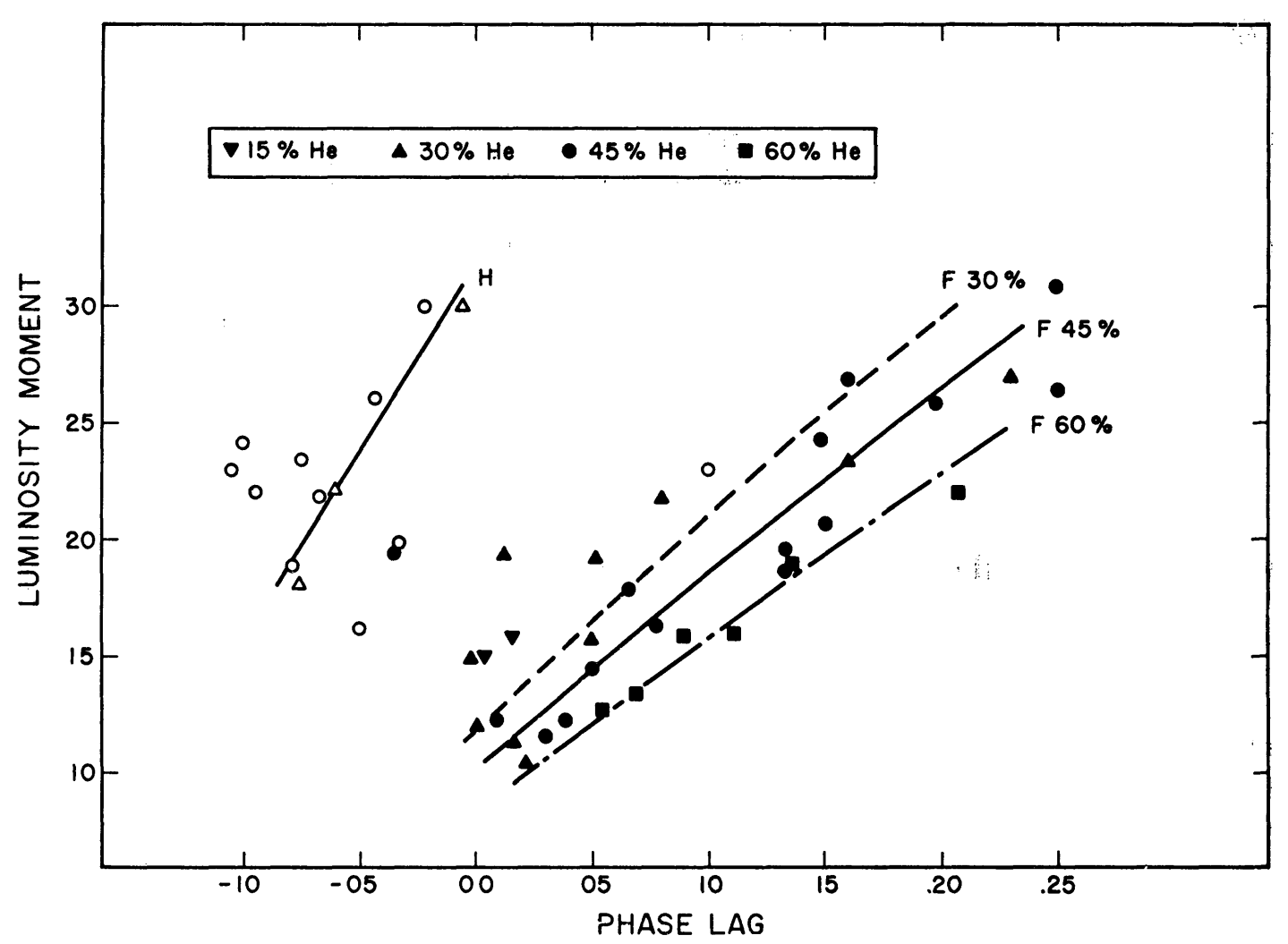

FIG. 13.-The correlation of the luminosity moment with the phase lag for the models of Table 3. Symbols are as in Fig. 9.

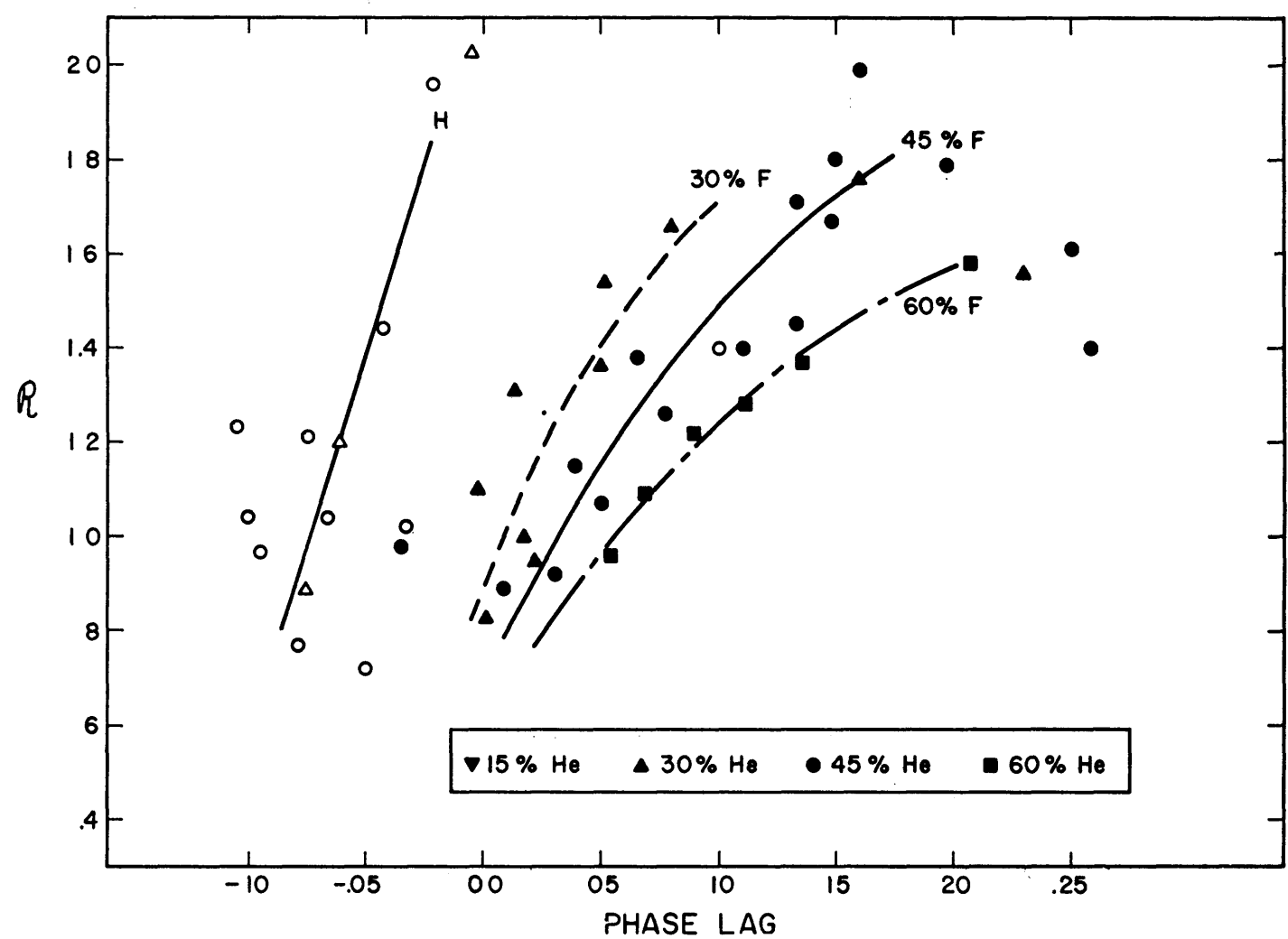

FIG. 14.- The correlation of the ratio $R$, of the radius amplitude to the integrated luminosity amplitude, with the phase lag for the models of Table 3. Symbols are as in Fig. 9. 
We have thus been able to find two independent quantities relating to phase lags and shapes in addition to the absolute amplitude. In examining the physical phenomena in the envelope, we can relate these quantities to the three quantities velocity amplitude, temperature amplitude, and phase relation of velocity and temperature. These three, of course, govern the luminosity amplitude and its phase and shape. We have chosen to use luminosity rather than temperature as a basic quantity because it is more readily observed and also was more precisely calculated. The measures of shape, relative amplitude, and phase lag we have discussed are not all independent and, at most, two of them can be used for independent measures. The magnitudes of the amplitudes are discussed next.

\section{b) The Full-Amplitude Survey}

Of the more than one hundred models which were examined for instability, fifty have been pursued until they approximated full amplitude and their light- and velocity-curves became almost periodic. For these models, which are listed in Table 3, it was then possible to arrive at reasonably accurate results for observable quantities related to the maximum amplitude or which are sufficiently amplitude-sensitive to be useful only when determined near maximum amplitude. In no case was there observed any systematic relaxation of the mean envelope structure in the course of the calculation. The mean radius (and mean $T_{e}$ ) of the final pulsating state in no case differed by as much as $\frac{1}{2}$ per cent from the initial static model. Such changes are in principle possible, but we have ignored them since they were not found in the relatively brief computer runs (never more than 50 periods of pulsation).

These models each involved from $\frac{1}{2}$ to 1 hour on an IBM 7094 compared to about 7 min for an investigation of instability. Thus, although it was essential to study a number of models pulsating at maximum amplitude, it was necessary to learn how to extract as much useful information as possible about the pulsation properties of a given model in a relatively short computing time. It was for this reason that the survey was in two parts dealing with medium-amplitude models and with maximum-amplitude models.

The basic purpose of both surveys was the same, namely, to learn how to interpret observational data on pulsation in terms of the physical characteristics of the models. Of course, the observed variables are certainly pulsating at their maximum amplitude so that our interest in smaller amplitude arises solely because it is computationally much easier.

We have already discussed the luminosity moment in the twelfth column of Table 3. We have, in addition, tabulated all of the amplitudes that it seemed possible to observe. These included the velocity, luminosity, effective temperature, radius, and integrated luminosity amplitudes. Finally, in the last three columns we have tabulated unobservable results referring to the driving mechanism, namely, the energy production in the hydrogen zone and the He II zone and the maximum kinetic energy of the envelope.

It was pointed out in discussing the phase lag that the velocities recorded here are the radial velocities (with respect to the star) of a fixed-mass layer in the star. These velocities are not to be compared to the observed Doppler velocities of spectral lines until the observed velocities are multiplied by some factor (conventionally $\frac{24}{17}$ ) to correct for the fact that only a projection on the line of sight is actually observed. There is a further difference between the computed and observed velocities arising from the fact that the observed velocities refer to a very shallow layer at $T_{e}{ }^{\max }$ and to a very deep layer at $T_{e}{ }^{\min }$. By reference to Figure 7 of Paper II or Figure 15 here, we see that at $T_{e}{ }^{\text {min }}$ there is essentially no velocity gradient in the atmosphere-it is almost all falling with the same velocity; except perhaps for the highest layers where the hydrogen lines are formed, the corrections to the observed velocities should be negligible at this time (corresponding to the time when the velocity is near its maximum value). At $T_{e}^{\max }$ however, there is a considerable velocity gradient; on the other hand, the atmosphere is 
very thin at this time so that the velocity differences between optical depths 0.1 and 0.2 are not large. In Figure 15, the velocity for zone 35 during the time between the points marked $X$ is distorted because the material is ionized and expanded to low density. Only for the hydrogen lines at very small optical depth would we expect a significantly higher peak velocity. We have considered a uniform mass depth which is somewhat deeper than the region of metal-line formation at this phase, and therefore our maximum outward velocities may be a slight underestimate of the observable metal-line velocities. Our calculations involved too crude a zone structure to be used for hydrogen-line velocities.

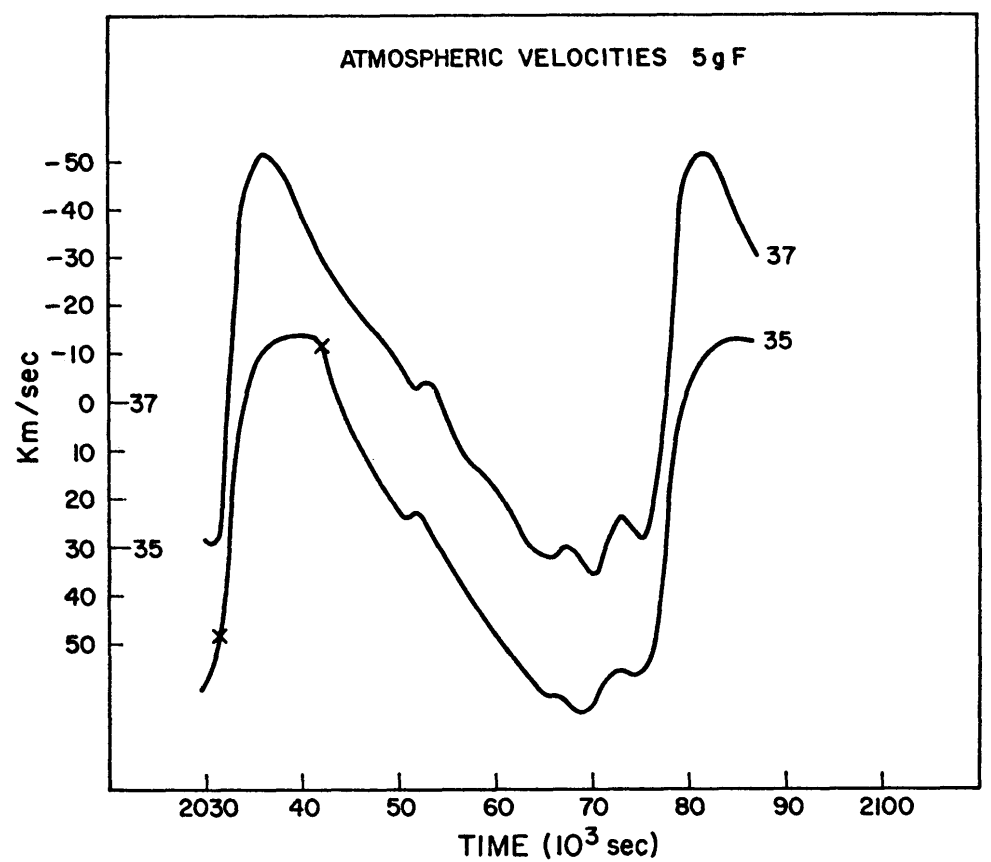

FIG. 15.-The velocities of two zones in the atmosphere of model $5 \mathrm{~g}$ F. The points $\times$ mark the times when zone 35 enters and leaves the ionized region.

\section{c) The Amplitude Variations}

Table 3 contains all of the results on the amplitudes of the steady-state pulsations which we have computed. The principal variation that we see in these results is the systematic change in amplitudes with $T_{e}$ for each of the sequences. The different sequences are very similar in this respect so we have chosen sequence 5 to represent the trends. These trends are shown in Figures 16-20, where the luminosity amplitude, $L_{\max }$ and $L_{\min }, T_{e}^{\max }$ and $T_{e}^{\min }$, velocity maximum and velocity minimum, and $\Delta R / R$ are shown as a function of $T_{e}$.

In general, the dependence of the amplitudes on mean $T_{e}$ is very similar to the dependences that have been noted in the globular-cluster variables (Roberts and Sandage 1955). The luminosity amplitude (Fig. 16) is about 0.5 mag. for the first-harmonic variables, increases to from 1 to $1.5 \mathrm{mag}$. for the highest- $T_{e}$ fundamental variables, and then decreases as $T_{e}$ decreases to about $0.5 \mathrm{mag}$. Where there are several first-harmonic variables in a sequence, the decrease in amplitude with decreasing $T_{e}$ is also apparent among them. The lowest-amplitude fundamental that has been calculated is about 0.5 mag. The maximum and minimum lumnosities in Figure 17 show the same general dependence. The principal part of the variation in amplitude is found in the variation of the maximum luminosity. The reason is, of course, that the maximum is peaked and is sensi- 


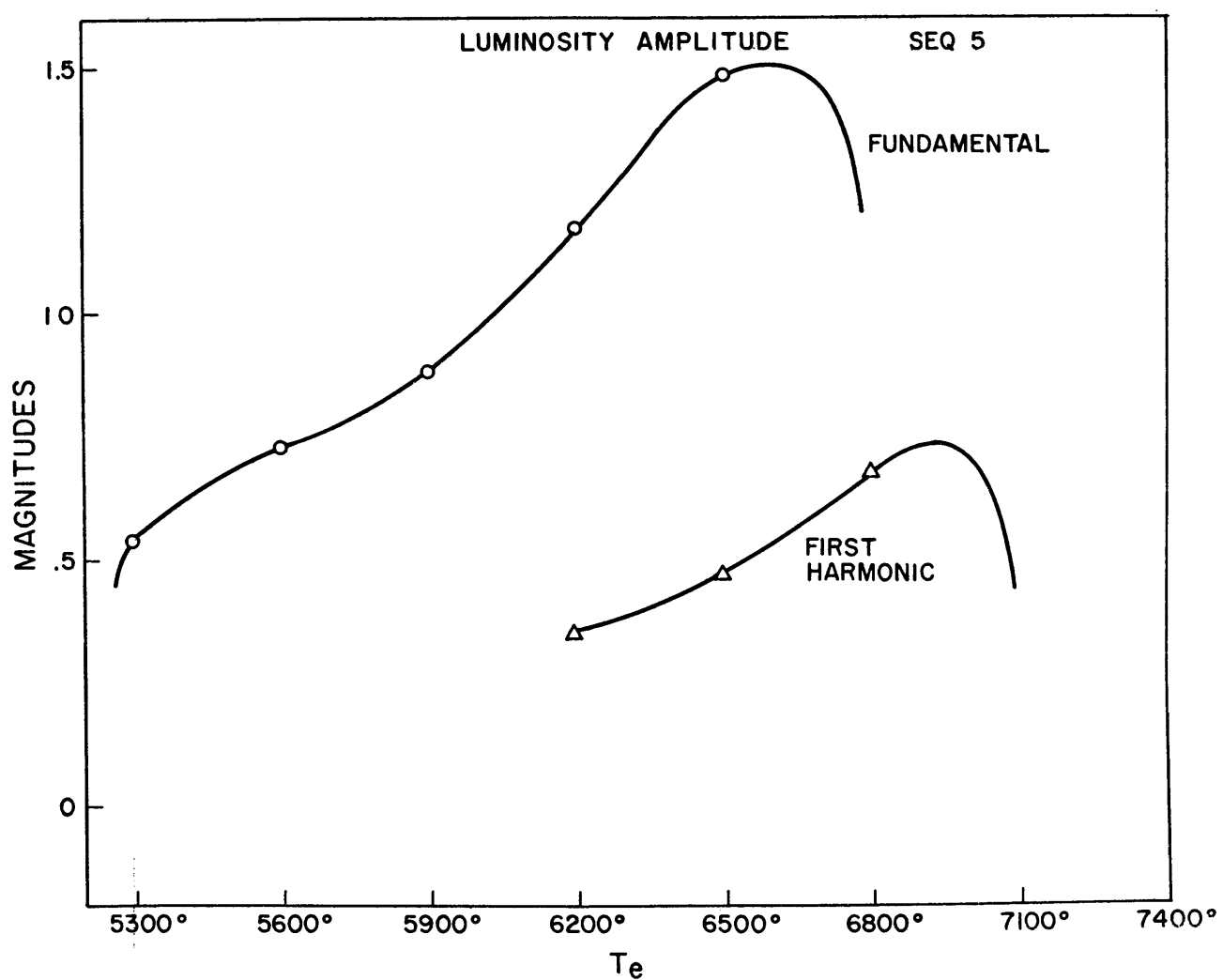

FIG. 16.-The variation of the luminosity amplitude with $T_{\boldsymbol{e}}$ for the models of sequence 5

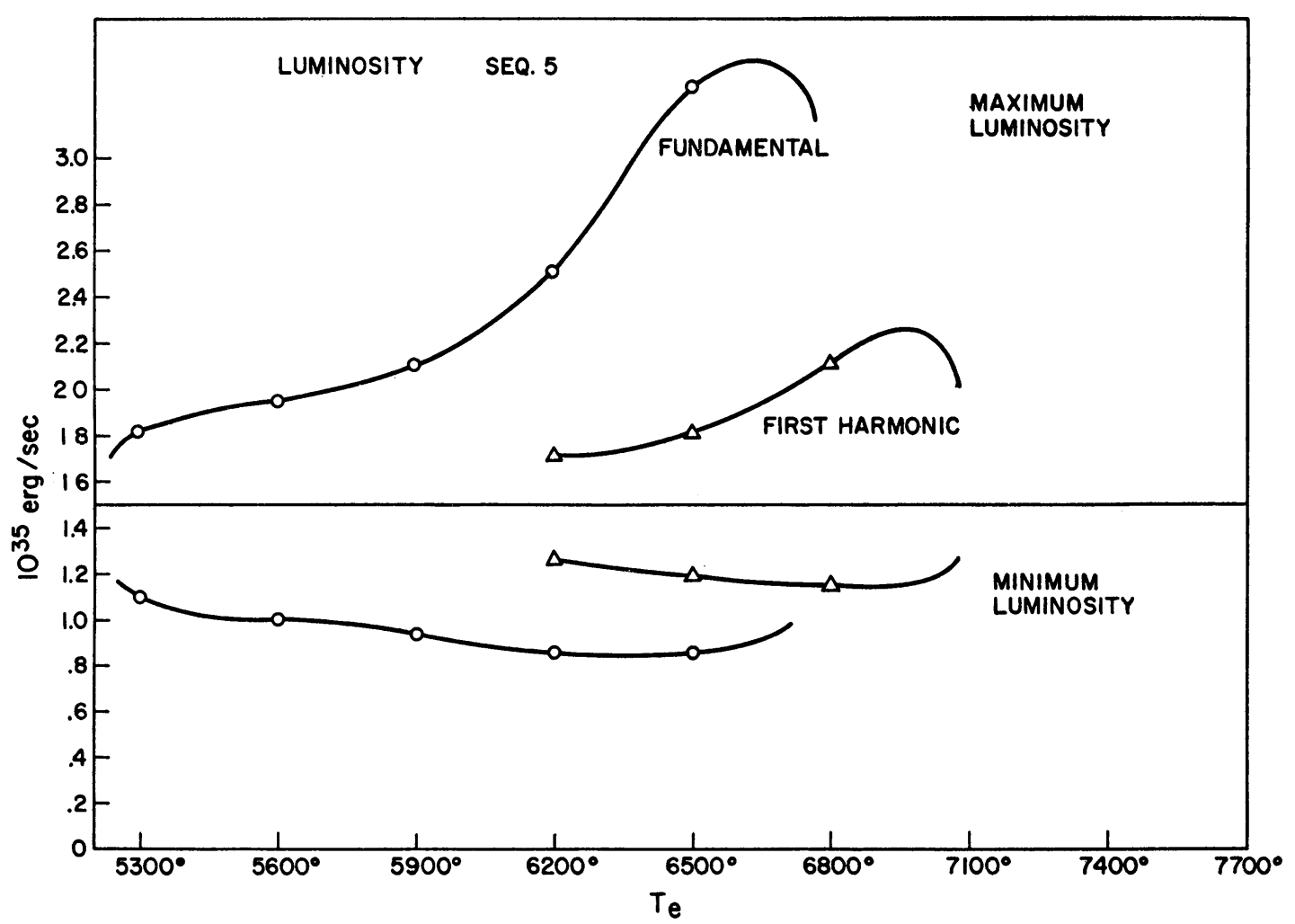

FIG. 17. - The variation of maximum and minimum luminosity with $T_{e}$ for the models of sequence 5 148 


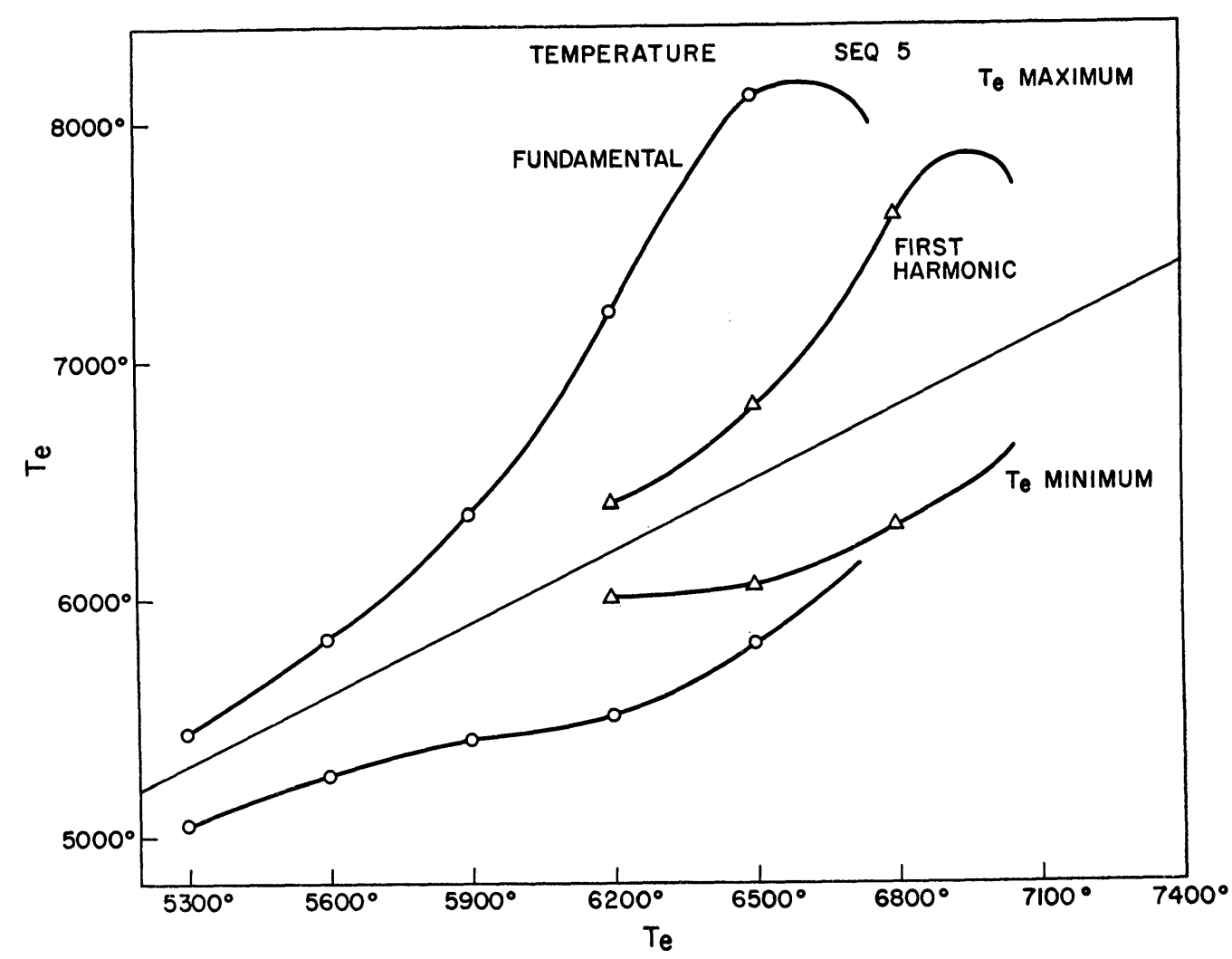

FIG. 18.-The variation of maximum and minimum $T_{e}$ with the mean $T_{e}$ for the models of sequence 5

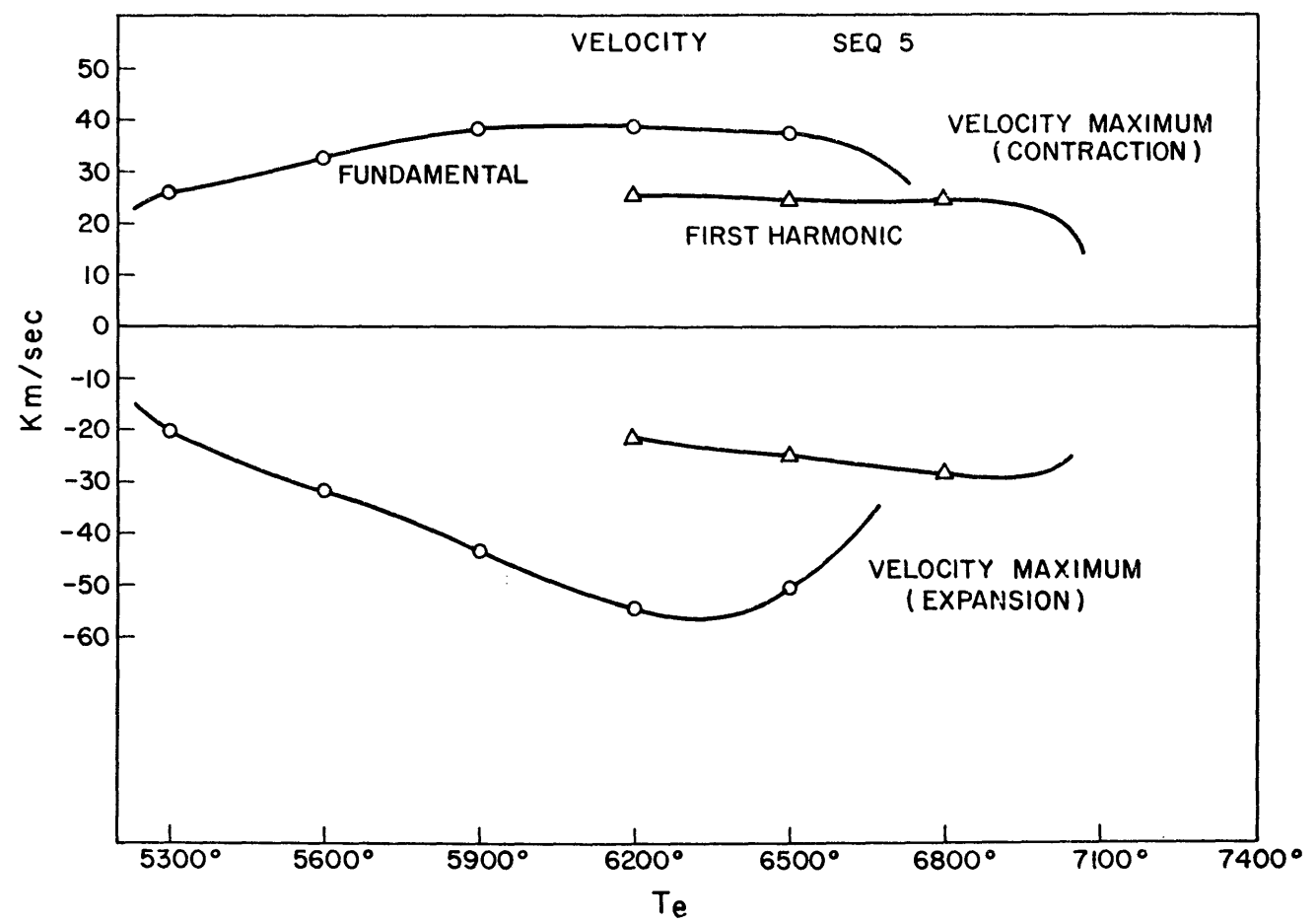

Frg. 19.-The variation of maximum and minimum velocity with $T_{\mathrm{e}}$ for the models of sequence 5 
tive to shape whereas the minimum is flat. Since the shape of the luminosity-curve has been seen to vary from peaked at higher $T_{e}$ to a rounded maximum at lower $T_{e}$, the maximum luminosity will systematically decrease toward lower $T_{e}$.

The maximum and minimum $T_{e}$ 's during a cycle are shown in Figure 18 as a function of $T_{e}$ mean. The same dependence shows here also. The first-harmonic pulsators have relatively small amplitudes, ranging from a high of $1000^{\circ}$ or more on the high- $T_{e}$ side to perhaps $500^{\circ}$ on the low- $T_{e}$ side. The amplitudes of the fundamental models are systematically higher, ranging from about $2000^{\circ}$ on the high- $T_{e}$ side down to $500^{\circ}$ on the low- $T_{e}$ side. This reduction in temperature amplitude with decreasing mean $T_{e}$ is, of course, intimately related to the systematic change in the shape and amplitude of the luminosity-curve with $T_{e}$. A consequence of the decrease in temperature amplitude with decrease in mean $T_{e}$ is that the value of the minimum $T_{e}$ during the period is only slowly

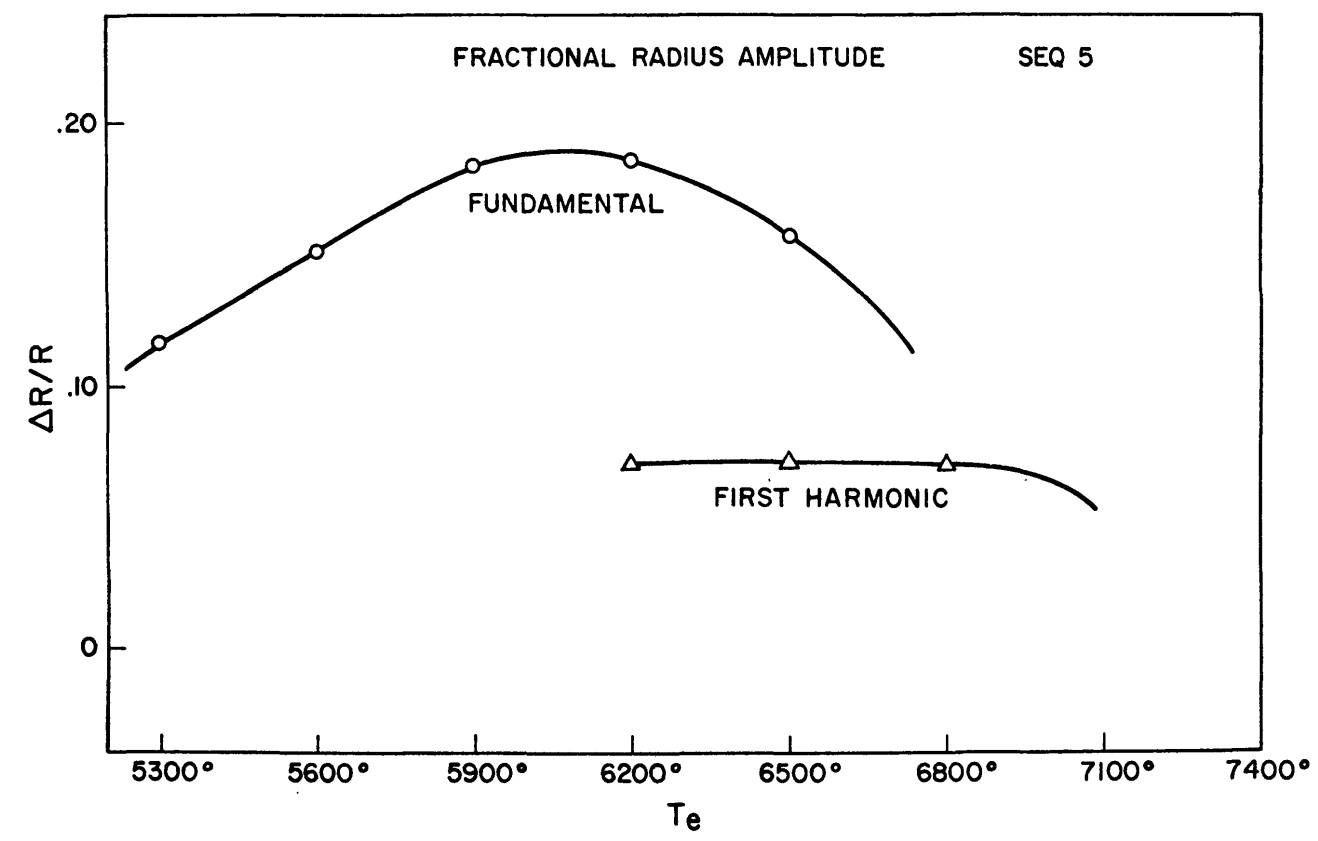

FIG. 20.-The variation of the radius amplitude with $T_{e}$ for the models of sequence 5

changing as $T_{e}$ mean is changed. Over a significant range in mean color, the value of $T_{e}{ }^{\min }$ is nearly constant. This also has been noted by observers (J. B. Oke, private communication).

The velocity amplitude also shows a systematic change with mean $T_{e}$. It is seen in Figure 19 that the velocity amplitude is also somewhat smaller for the first harmonic and in general decreases toward lower $T_{e}$. However, because of a systematic change in the shape of the velocity-curve with $T_{e}$, the negative amplitude exceeds the positive amplitude for higher $T_{e}$ whereas the reverse is true for the lower $T_{e}$. Observationally, this means that the $\gamma$-velocity is more positive than the mean of $V_{\max }, V_{\min }$ for the higher- $T_{e}$ fundamental variables and the $\gamma$-velocity is less positive than the mean of $V_{\max }$ and $V_{\min }$ for the lower- $T_{e}$ fundamental variables. It is also a consequence of those variations that the positive amplitude of the fundamental is largely independent of $T_{e}$, whereas the negative peak is rapidly changing with $T_{e}$.

The amplitude that shows greatest constancy is the fractional radius amplitude $\Delta R / R$ (where $\Delta R$ is the full amplitude). This is, in general, near 0.08 for the first harmonic and is near 0.16 for the fundamental. An approximate constancy of this amplitude at these values can easily be understood in terms of a picture of the limiting velocity- 
curve as a sawtooth-curve with a sudden velocity reversal followed by a linear change between maximum and minimum. This picture also would give a radius variation consisting of parabolas connected at cusps. If the linear-velocity change involves a constant acceleration of some fraction $a$ of the local $g$, we can calculate $\Delta R=\frac{1}{2} a g P^{2} / 4$, where $P$ is the period. Then

$$
\frac{\Delta R}{R}=\frac{1}{8} a \frac{6.67 \times 10^{-8}}{R^{3}} M P^{2}
$$

If $P$ is in days, this gives

$$
\frac{\Delta R}{R}=2.61 a(P \sqrt{ } \rho)^{2}
$$

Since we find the atmospheric acceleration is usually near $0.4 \mathrm{~g}$, this gives $\Delta R / R \approx 0.18$ for the fundamental, and about $\Delta R / R \approx 0.09$ for the first harmonic. Thus we can understand the values we find for $\Delta R / R$ in terms of a maximum-amplitude motion which is associated with a sawtooth velocity-curve in which the acceleration is about $\frac{1}{2}$ of the local value of $g$. We will show in a later section that the limiting amplitude is associated with the sudden velocity reversal.

\section{d) The Energy Production}

The sources of excitation and dissipation of the pulsation have been investigated as in Paper II. The work per period in each mass zone was found from $\varnothing P d V$ evaluated in most cases near the maximum amplitude where the motion was most nearly periodic. In a few cases (sequence 5), the work integral was also evaluated at the intermediate amplitude (about $\frac{1}{2}$ maximum) at which the motion was initiated. As was discussed in Paper II, the difficulties associated with treating radiation flow in the hydrogen zone near $10^{4}{ }^{\circ}$ did not permit a truly small amplitude investigation of the energy production. It would have been possible to evaluate the energy in the helium zone at arbitrarily small amplitude, but, since it behaves linearly up to $\frac{1}{2}$ maximum, it was unnecessary.

The zone that shows great non-linearity at very small amplitude is the hydrogen zone. This zone probably shows a linear behavior at very small amplitude when the ionization front moves through only a fraction of its thickness in one period. The limiting amplitude of this linear behavior is very sensitive to convection, which thickens the front. A second, different linear region then results when the front moves through a distance large compared to its thickness, but the amplitude is still below maximum and shock waves or very steep velocity fronts are not present. It is this latter linear region which we have investigated. Finally, steep velocity fronts form, and the amplitude reaches a limiting value for reasons to be discussed later.

We chose to divide the energy production, somewhat arbitrarily, into three regions: a hydrogen zone, a helium zone, and a dissipative region. These can be understood by referring to Figure 21, where the work developed per period in each zone is shown in percentage of the total kinetic energy. In general, the dissipative region contains both an atmospheric part and a deep part. The region between these dissipative regions is found to have two maxima which are somewhat arbitrarily separated into a contribution due to He II and a hydrogen zone. If there is negative work in the minimum between the two peaks, it is included also as part of the contribution of these zones. The total of the energy developed per period in the $\mathrm{H}$ zone is shown in the thirteenth column of Table 3 and the total in the helium zone is given in the fourteenth column. In each case, the energy production is divided by the total kinetic energy given in the fifteenth column so that the entries in the thirteenth and fourteenth columns are fractional contributions to the kinetic energy of the envelope per period. These units are the same as those in the eleventh column of Table 2 except that the energies there are expressed in per cent. The 
net energy increase or decrease in Table 2 is shown for medium-amplitude models, whereas the results in Table 3 are for maximum-amplitude models where the total dissipation just equals the total energy developed per period.

The dependence of energy production and dissipation on $T_{e}$ for a typical sequence (5) is shown in Figure 22. The medium- or small-amplitude results are shown for stable as well as unstable models and for first-harmonic and fundamental modes. At the high$T_{e}$ boundary, the energy production in the helium zone is sharply decreasing; however, in the transition region between first harmonic and fundamental, both modes are unstable and the high-temperature boundary of the fundamental instability has not been explored

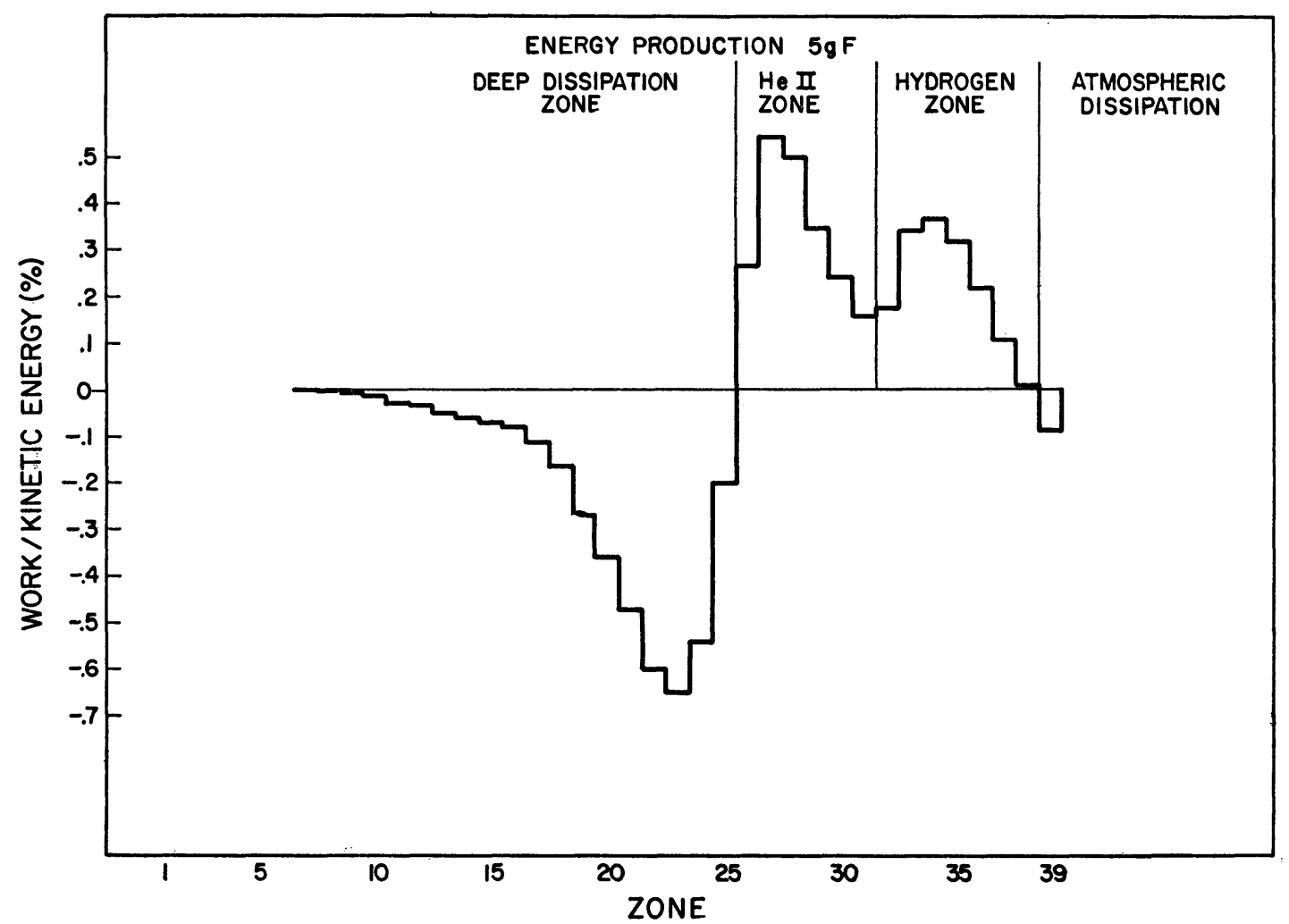

FIG. 21. - The energy production and dissipation at various depths in model $5 \mathrm{~g} \mathbf{F}$

since the motion changes to the harmonic. At the lowest temperatures, the energy production in both hydrogen and helium are rapidly decreasing whereas the dissipation is increasing.

Table 3 shows a number of trends in the energy production. If we examine a series of models with fixed $L, M, T_{e}$, and varying composition, we see that the total energy production rate (and, of course, dissipation) decreases as the helium content is increased from 0 to 45 or 60 per cent. At the same time, the contribution from hydrogen decreases and the contribution from helium increases even more rapidly than the helium content. In the first-harmonic models, since the amplitude decreases more rapidly toward the node, I had expected the relative contribution of the helium zone to be reduced relative to the hydrogen zone. Just the opposite result is shown by the calculations. The region of the first-harmonic models below the node (which occurs about zone 15) is effectively inert. The amplitude is so small that the system would behave almost the same if an immovable boundary with constant luminosity were placed at the node.

The total energy content of the envelope increases rapidly with increasing stellar mass 
at fixed luminosity because of the increase in mass of the outer envelope. For this reason, the ratio of the work developed per period to the kinetic energy is greatest for small masses. The total work developed per period is more or less independent of the mass for fixed luminosity but increases with increasing luminosity so that the efficiency (work per period/luminosity $\times$ period) is more or less independent of mass and luminosity in the fundamental. The kinetic energy of the envelope is largely independent of luminosity for fixed mass; as a consequence, the fractional work developed per period is greater the greater the luminosity for fixed mass. However, if the period is held constant, the fractional work per period decreases with increasing mass and luminosity. The kinetic

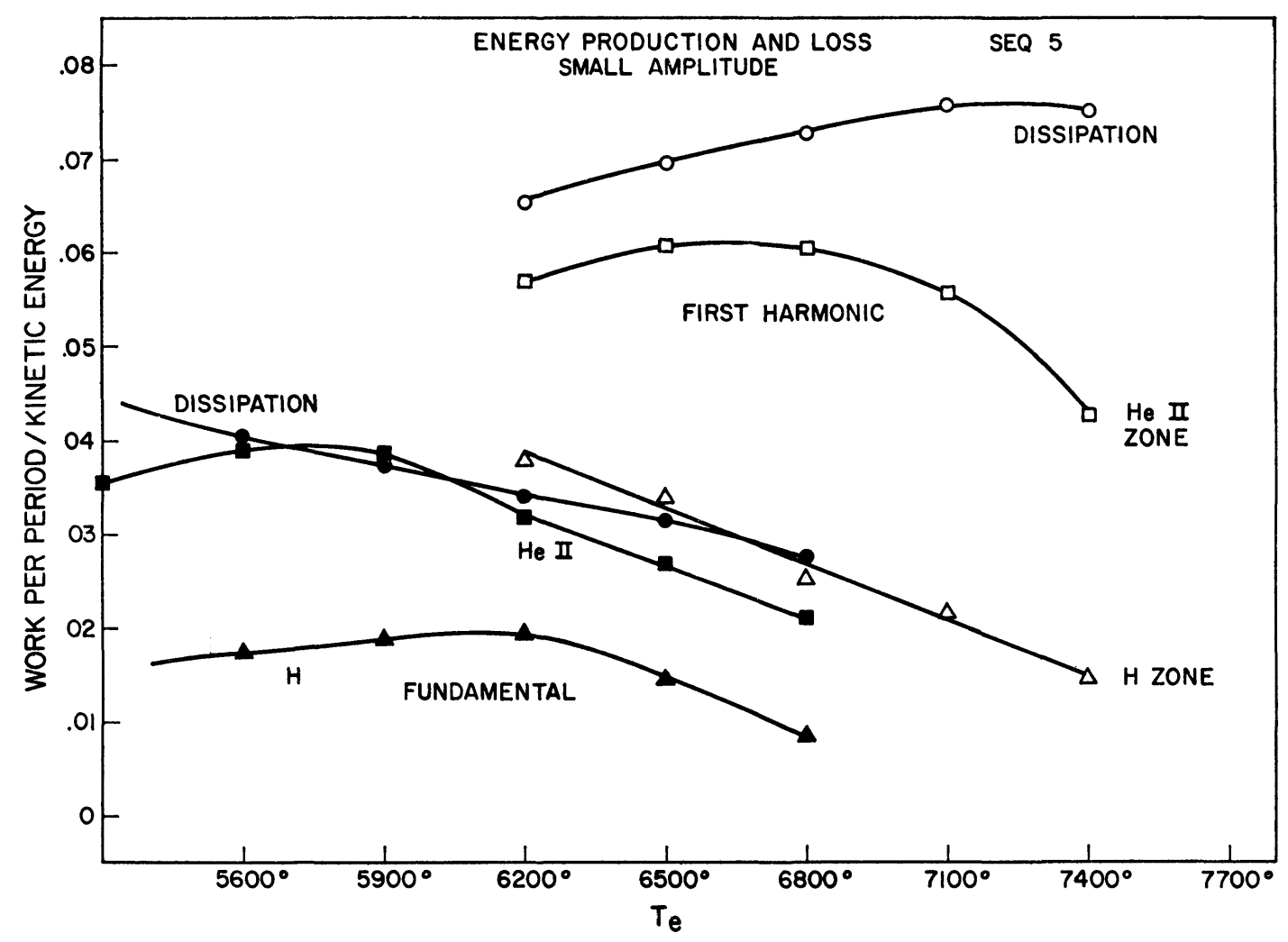

FIg. 22.- The variation with $T_{e}$ of the ratio of energy production and loss to the kinetic energy for the models of sequence 5 .

energy in the first harmonic is only about 10 per cent of that in the fundamental; for this reason, although the efficiency is greatly reduced in the first harmonic, the fractional work developed per period is larger in the first harmonic. The fractional work per period is of particular interest in the calculations because it is related to the net fractional increase in amplitude per period at small amplitudes, and the success of the computations requires that the fractional increase in amplitude per period be not too small. Also the violence of the pulsation and the depth at which shock waves are developed is also related to the fractional increase of amplitude per period. The larger the increase at small amplitudes, the more violent the pulsation at maximum amplitude and the deeper are the shock waves developed.

\section{e) The Limiting Amplitude}

In the course of these calculations, the limiting amplitude has been encountered in a great variety of ways. Sometimes it was approached from smaller amplitudes and some- 
times from larger amplitudes. In some models, the amplitude was started above the limiting amplitude and decreased rapidly to slightly below and then increased again to the limiting amplitude. This large amount of qualitative experience with the behavior of these systems in the neighborhood of their maximum amplitude led to the conclusion that the development of steep velocity fronts is always associated with the cessation of the growth of the amplitude. This feature of the final motion has previously been called on in discussing the magnitudes of $\Delta R / R$ to show why they attain the values about 0.16 in the fundamental and 0.08 in the first harmonic.

In order to explore the causes underlying the amplitude limitation, we have made a thorough study of one particular model $-5 \mathrm{~g} \mathrm{~F}$, which is a typical fundamental pulsator.

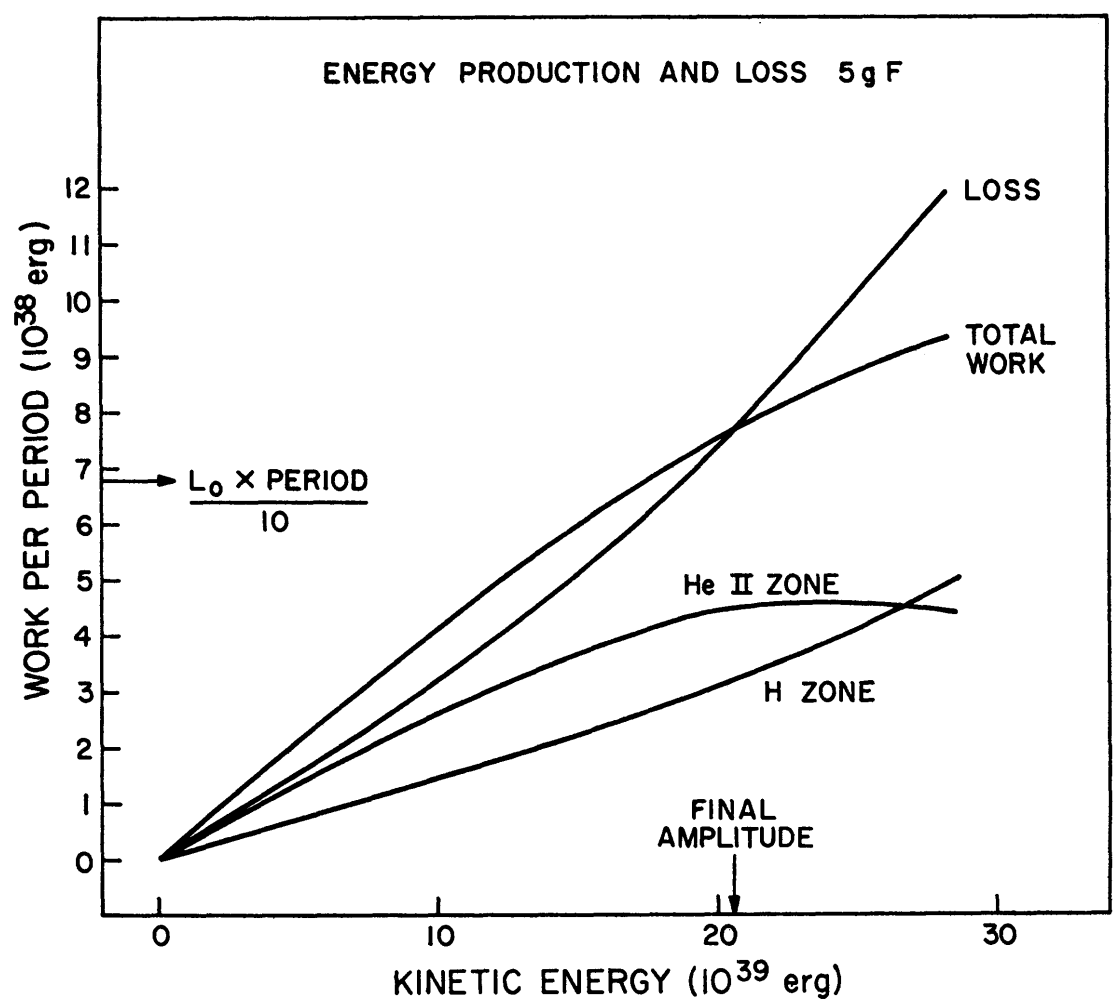

Fig. 23.-The variation of the energy production and loss with kinetic energy for model $5 \mathrm{~g} \mathrm{~F}$

This model was initiated at medium amplitude (later referred to as "small amplitude"); the amplitude was then driven artificially to a value exceeding the limiting value (referred to as "large amplitude") and then brought to the limiting amplitude. In this way the motion was studied over a considerable amplitude range including the limiting amplitude.

The general behavior of this model was similar to that of the other fundamental pulsators. The fractional increase of kinetic energy per period was found in all the models to maintain an approximately constant value until the radius amplitude $(\Delta R / R)$ was about one-half of its limiting value, and then it decreased to zero at the limiting value. The increase continued to become negative at amplitudes exceeding the limiting one. The variation of the fractional increase of kinetic energy during the approach to the limiting amplitude is displayed in Figure 8 of Paper II.

The final distribution of energy production in $5 \mathrm{~g} \mathrm{~F}$ is shown in Figure 21. The totals of the work developed in the hydrogen and helium zones and the total dissipation were then studied for various amplitudes, and the results are displayed in Figure 23. It was 
immediately apparent that the principal non-linearity appeared as a relative reduction in the work developed by $\mathrm{H}$ II at large amplitudes, and it could be stated that this reduction was responsible for limiting the amplitude. On examining individual zones, it became clear that the largest reduction was in zones 28 and 29.

The question next examined was how this "saturation" of energy production by helium was related to the observation that steep velocity fronts are associated with the limiting amplitude. The development with increasing amplitude of this steep velocity front at zone 28 is shown in Figure 24, where the ratio of peak minus mean pressure to velocity peak is shown as a function of energy amplitude. When the motion is sinusoidal,

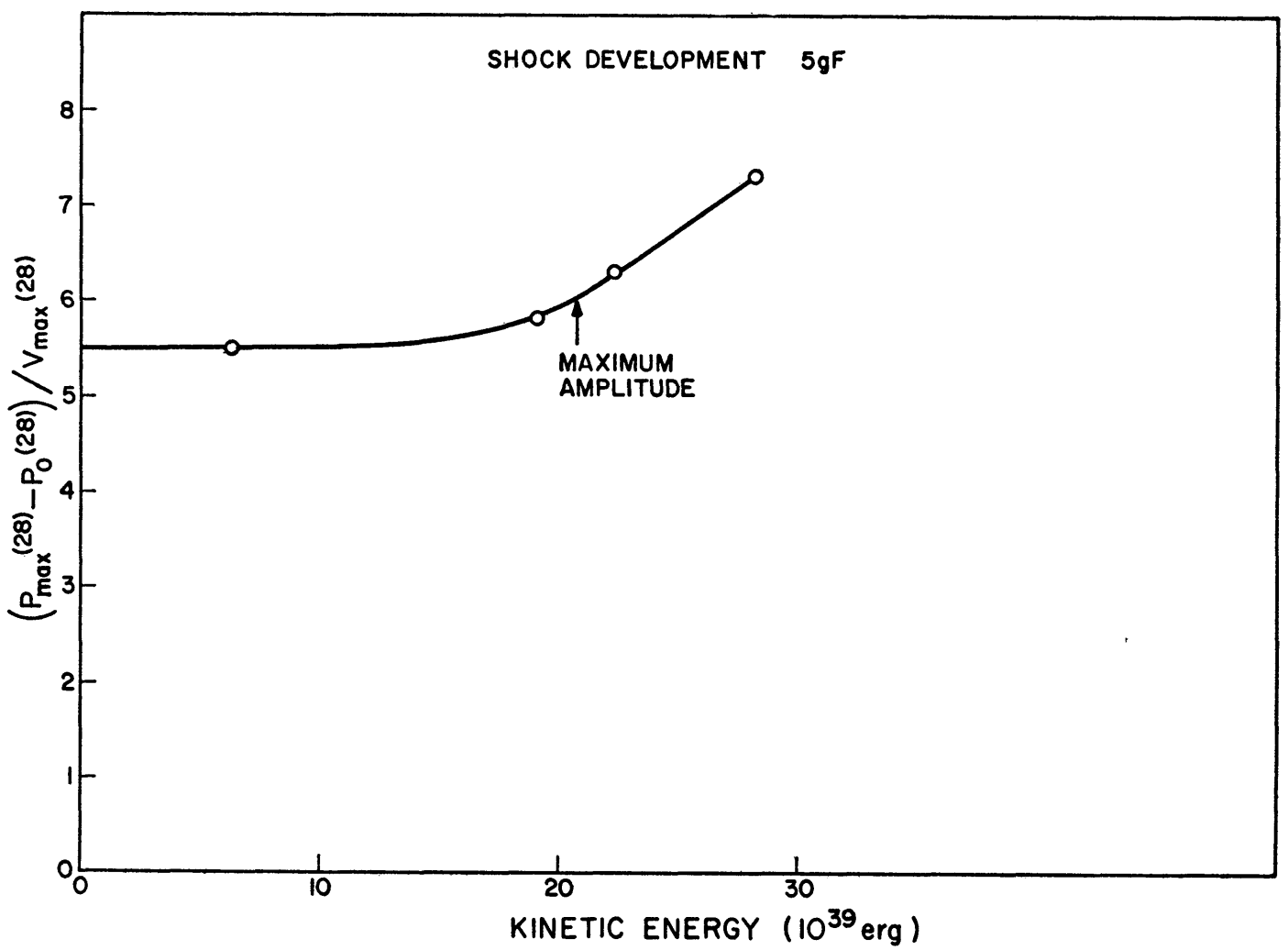
$5 \mathrm{~g} \mathrm{~F}$.

FIg. 24.-The development of excessive pressures at large amplitude in the helium zone for model

the difference of the peak pressure and the static or mean pressure at a zone is proportional to the peak velocity attained during the period. On the other hand, when the velocity front becomes steep, the accelerations become very large and the peak pressure grows more rapidly. It is apparent that the maximum amplitude is reached after the pressure peak has begun to become abnormally high associated with steepening of the velocity fronts. The velocity-curves are shown for large amplitude in Figure 25 . It is again apparent that it is just in the helium zone (zones 26-31) that the velocity-curves first develop the characteristic steep front that then persists in the hydrogen zone.

In order to see in more detail how this abnormally high pressure peak in the helium zone is responsible for limiting the amplitude, we have examined the luminosity as a function of time at various depths in the star both at small amplitude (Fig. 26) and large amplitude (Fig. 27). In Figure 26 the luminosity peak is approximately symmetrical with respect to maximum compression in zone 26 and below. This is the "quasiadiabatic" behavior characteristic of the dissipating regions. Zones 27-31 are where 
He II ionization is relevant. They show the characteristic phase delay of the luminosity peak which means that energy is being absorbed in the neighborhood of maximum density. This is, of course, a work-producing relation. A further sizable phase delay and work production occurs in the hydrogen zone (zones 32-37). In Figure 27, at large amplitude, we see immediately that a new, early peak in the luminosity has appeared just before maximum compression and the phase delay of the luminosity is largely eliminated in the helium zone by this peak. The luminosity peak in the hydrogen zone is still delayed well after maximum compression so that that zone is still functioning as a heat engine as we already saw.

We can now see the connection of the high-pressure peak to the "saturation" or loss of effectiveness of the $\mathrm{He}$ II zone. To clarify this, we show the opacity at various depths as a function of time for small amplitude in Figure 28 and large amplitude in Figure 29. These figures show immediately the difference between the energy-producing and energy-

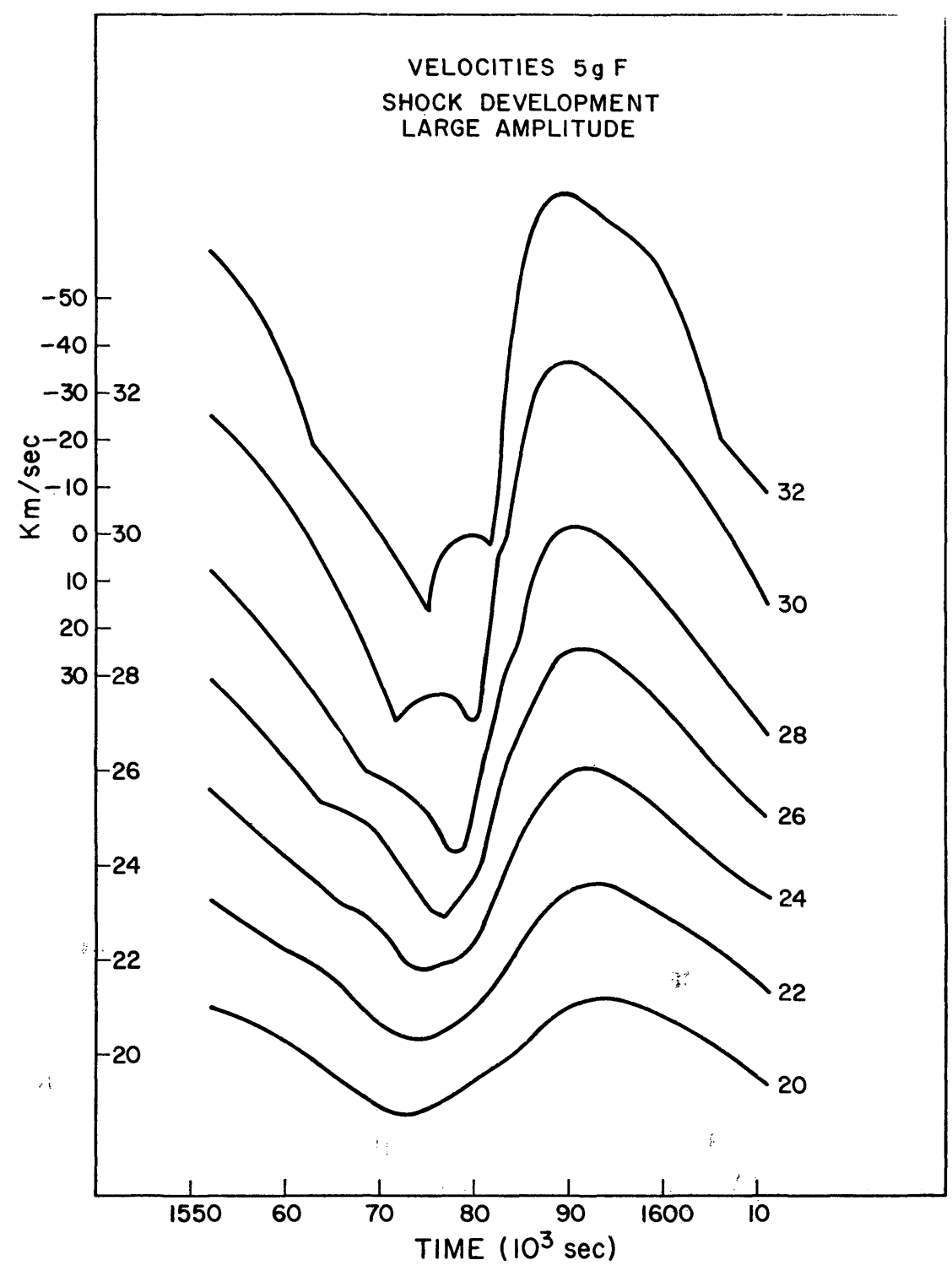

FIG. 25.-The steepening of the velocity front in the helium zone (zones 26-31) for a large amplitude pulsation in model $5 \mathrm{~g}$. 
dissipating zones in terms of the so-called opacity mechanism. In the dissipation region (zones 25 and below), we see that the opacity has the normal behavior and is a minimum at maximum compression, leading to dissipation. In the $\mathrm{He}$ II zone, however, because of the small temperature increase associated with compression, the opacity reverses its behavior and achieves a peak at maximum compression at zone 30. This, in turn, leads to the energy production of this zone. This peak at maximum compression persists in the hydrogen zone. However, at large amplitude, we can see a significant change in the behavior at zones 28,30 , and 32 . The large peak pressure in these regions at maximum compression, arising from the steep velocity front, has driven the temperature so high that the conventional decrease in opacity with increasing temperature gives a severe reduction in opacity just at maximum compression and thereby reduces the energy production in the He Ir zone. This effect does not greatly reduce the opacity in the hydrogen zone, which continues to energize strongly.

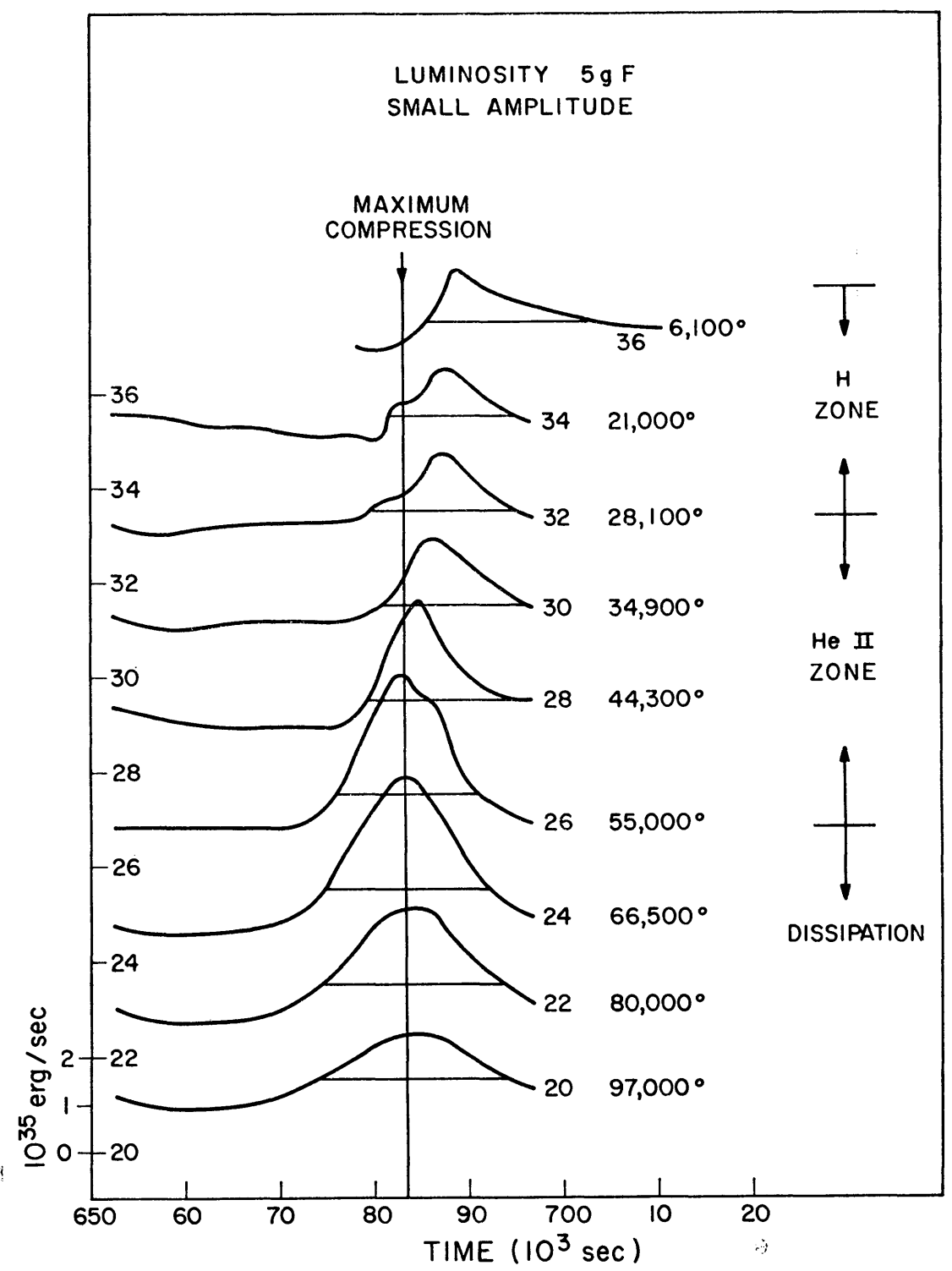

FIg. 26.-The diminution in amplitude and progressive shifting of phase of the luminosity in going from the dissipation region to the photosphere. Model $5 \mathrm{~g}$ F at mẹdiụm amplitude, 
We have seen, then, how the development of a steep velocity front in the helium zone is able, through the conventional opacity law, to reduce the energy production in this region and thus lead to the amplitude limitation. At the same time we can see why other characteristic effects have been noticed as the amplitude increased. We saw in Table 2 of Paper II that the phase lag showed a characteristic decrease, and also we have seen in this survey that the luminosity-curve becomes characteristically steeper in its front as well as lagging less at large amplitude. This steepening is related to the early peak that develops in the luminosity due to the opacity drop at maximum compression in the helium zone.

A further non-linearity in the helium zone is apparent in Figure 30 and is discussed in the Appendix. The opacity of hydrogen shows a shelf or bump in the region $\log T \approx 4.6$ which happens to coincide with $\mathrm{He}$ II ionization. For small amplitudes, the helium zone occurs where the opacity is nearly temperature-independent, but at large amplitudes the temperature of these zones rises above the shelf and a large drop in opacity occurs.

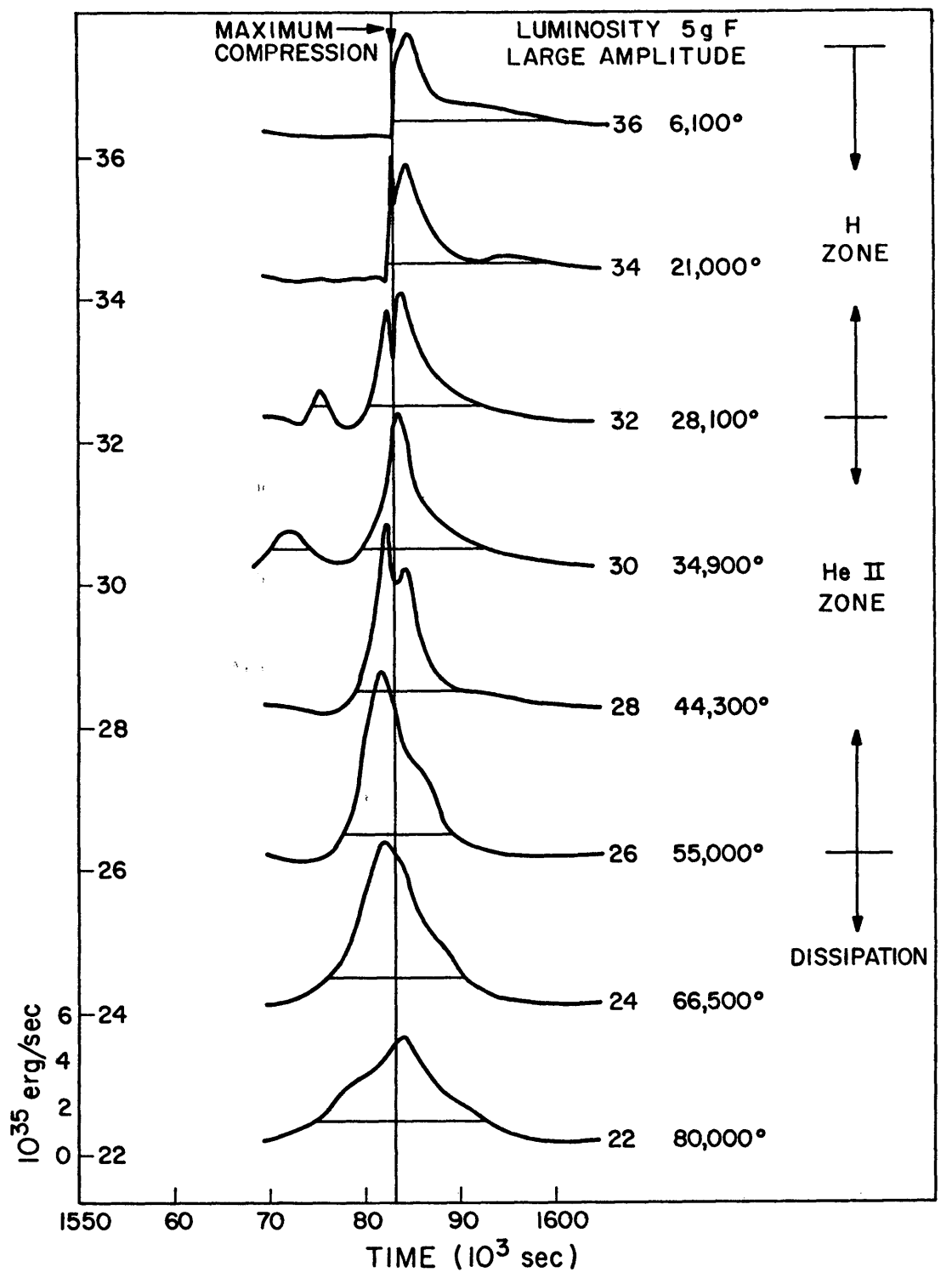

FIG. 27. - The luminosity at large amplitude in model $5 \mathrm{~g}$ F, showing the early peak in the helium zone, 
This discussion shows that the amplitude limitation results from a rather complex interplay of non-linearities - the non-linearity associated with incipient shock development interacting with the non-linearity associated with the finite heat capacity of helium ionization and the non-linearity of the opacity law. It is possible that other models that we have not examined carefully may show additional features. However, the steep velocity-front limitation seems characteristic of all of them.

\section{COMPARISON WITH OBSERVATION}

\section{a) The Shapes of Light-and Velocity-Curves}

We present in Figures 31-35 a considerable number of the calculated light- and velocity-curves in order to be able to discuss various features that correlate with observation. Figures 31-33 show both light- and velocity-curves with the proper phase relations. The light-curve is above and the corresponding velocity-curve just below in the usual astronomical sense. Figures 34 and 35 show a collection of velocity-curves.

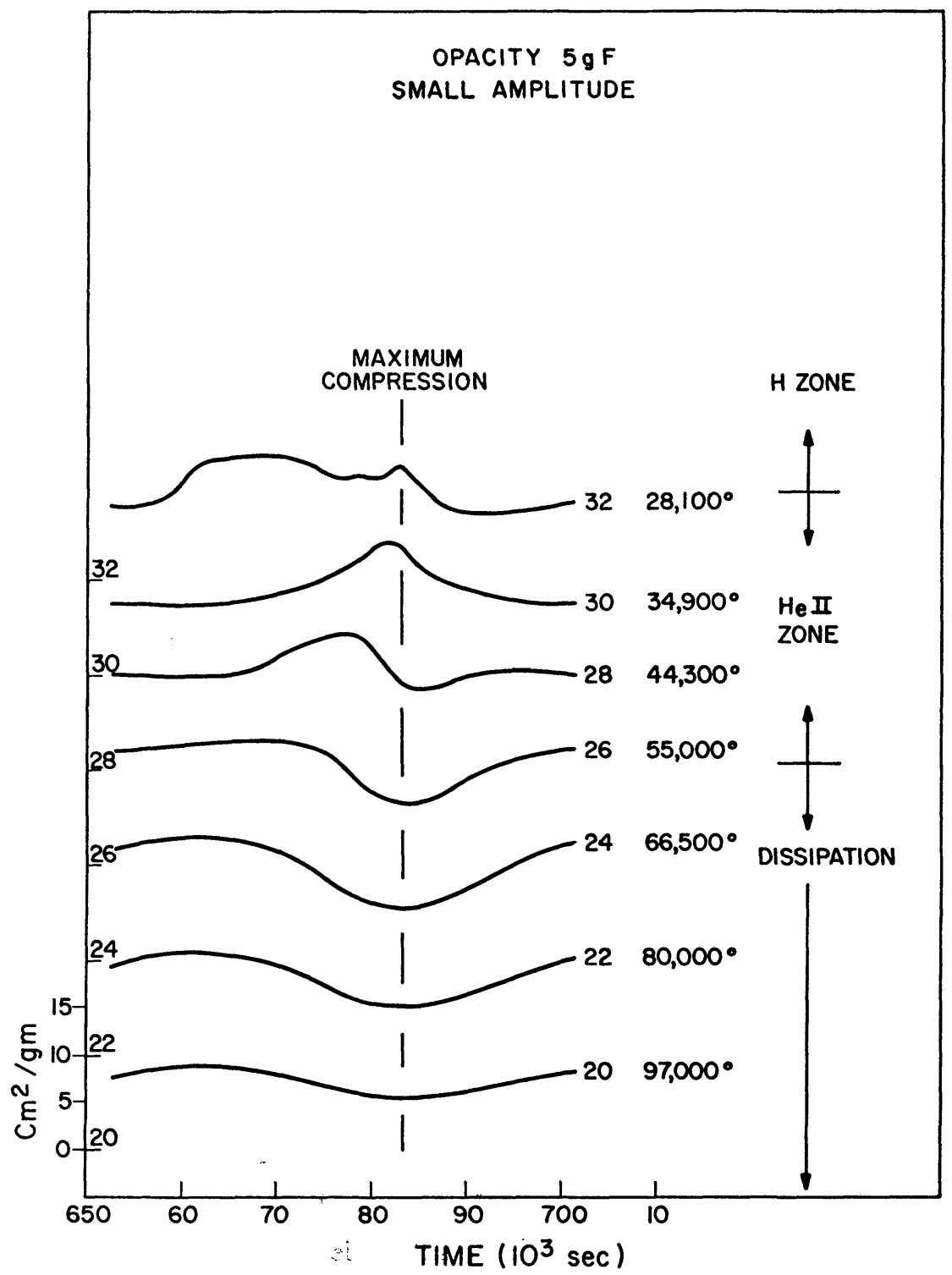

FIG. 28. - The behavior of the opacity in the dissipating region and in the helium zone for model $5 \mathrm{~g} \mathrm{~F}$ at medium amplitude. 
The most prominent feature of the first-harmonic light-curves in Figure 33 is the preliminary peak in luminosity occurring at a time prior to the time of velocity reversal or maximum compression. This peak is followed by a dip that is coincident with the velocity reversal and a subsequent rise to a second peak again. A very similar dip, although not as prominent, is clearly evident in observed type $c$ light-curves ${ }^{2}$ such as DH Peg (Tifft 1964) and T Sex (Preston and Paczyński 1964) and also in light-curves of type $c$ variables in $\omega$ Centauri (Martin 1938). We believe the feature we have calculated is the same as that observed and therefore have examined the calculation with care in an attempt to discover the cause of the feature.

In this investigation, we found that the early rise in luminosity (before maximum

${ }^{2} \mathrm{I}$ am indebted to G. W. Preston for drawing my attention to this feature of observed light-curves and for a generally illuminating discussion on a number of other questions related to RR Lyrae stars.

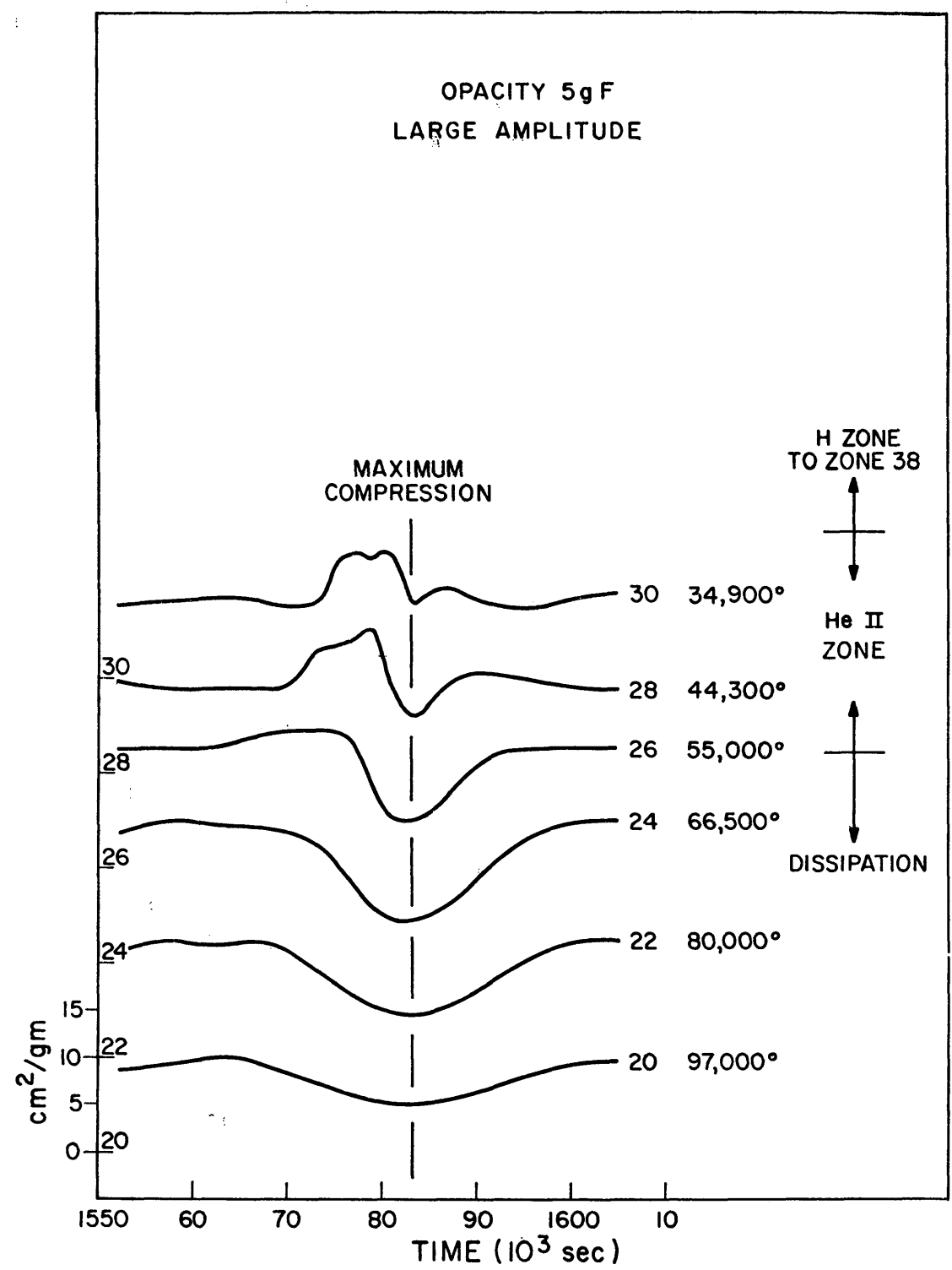

FIG. 29.-The behavior of the opacity at large amplitude, showing the minima at maximum compression in the helium zone. 


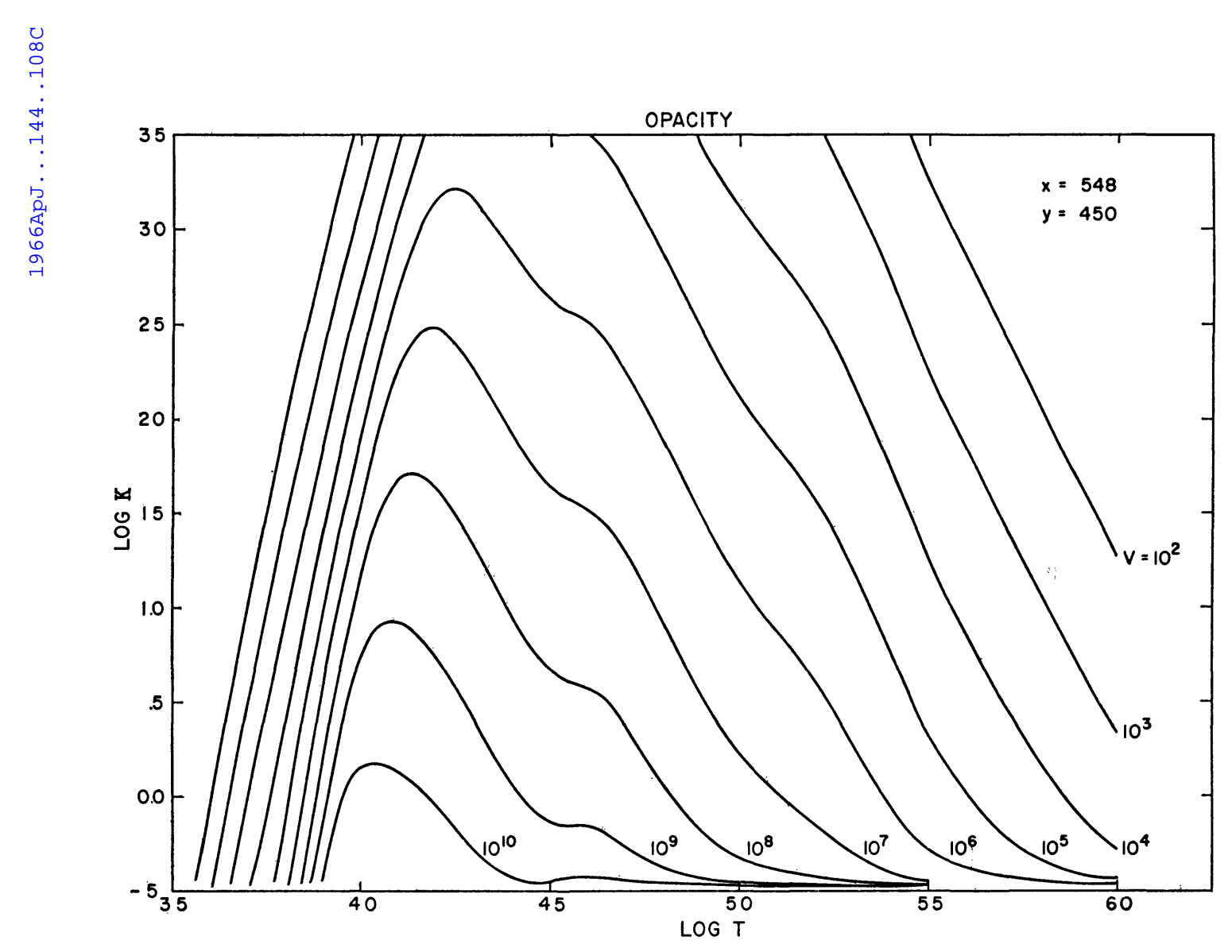

FIG. 30.-The variation of the opacity with temperature and specific volume for a mixture with 45 per cent helium and $Z=0002$.

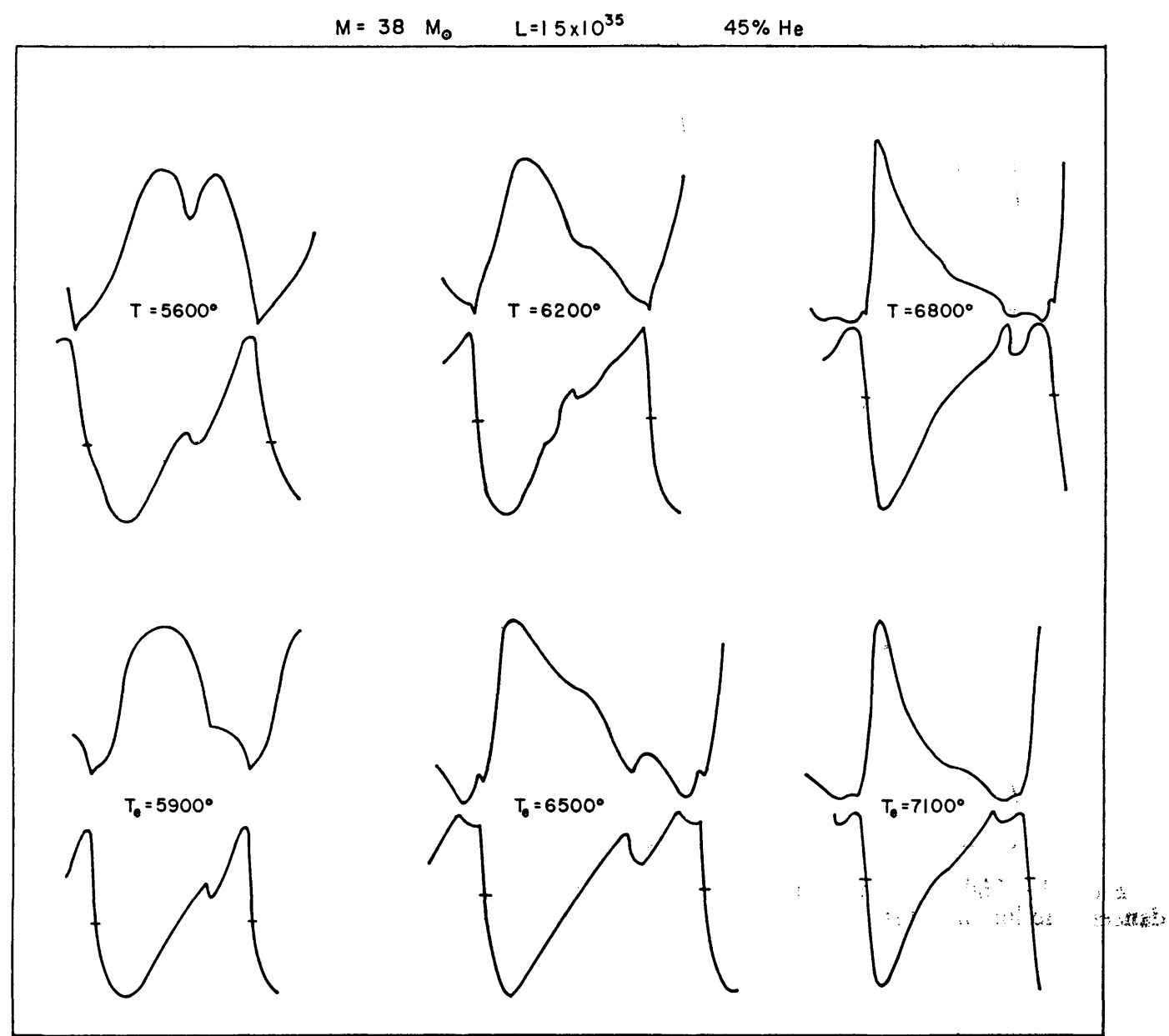

Fic. 31. - Light- and velocity-curves for the fundamental mode for sequence 8. In each case the veloc-

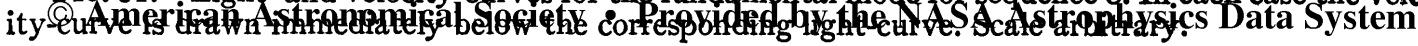


compression) was part of a general trend for the rise in luminosity to occur progressively later as one goes from variables on the high- $T_{e}$ side to the low- $T_{e}$ side of the instability strip (see Figs. 31-33). Now for an adiabatic pulsator the maximum luminosity will coincide with maximum compression, so the rise of luminosity will precede maximum compression by 0.25 period. However, the adiabatic pulsator is stable, and we found (Table 2) that the highest $T_{e}$ at which the instability is found occurs with a significant ionization heat capacity of the ionizing layers that can delay the flux rise by about 0.15 period. It is thus qualitatively consistent that the earliest luminosity rise of unstable stars precedes maximum compression by about 0.1 period. We have also seen that for suitable masses the unstable stars on the high- $T_{e}$ side of the instability are first-harmonic pulsators, and therefore the early rise in luminosity of these stars is an understandable part

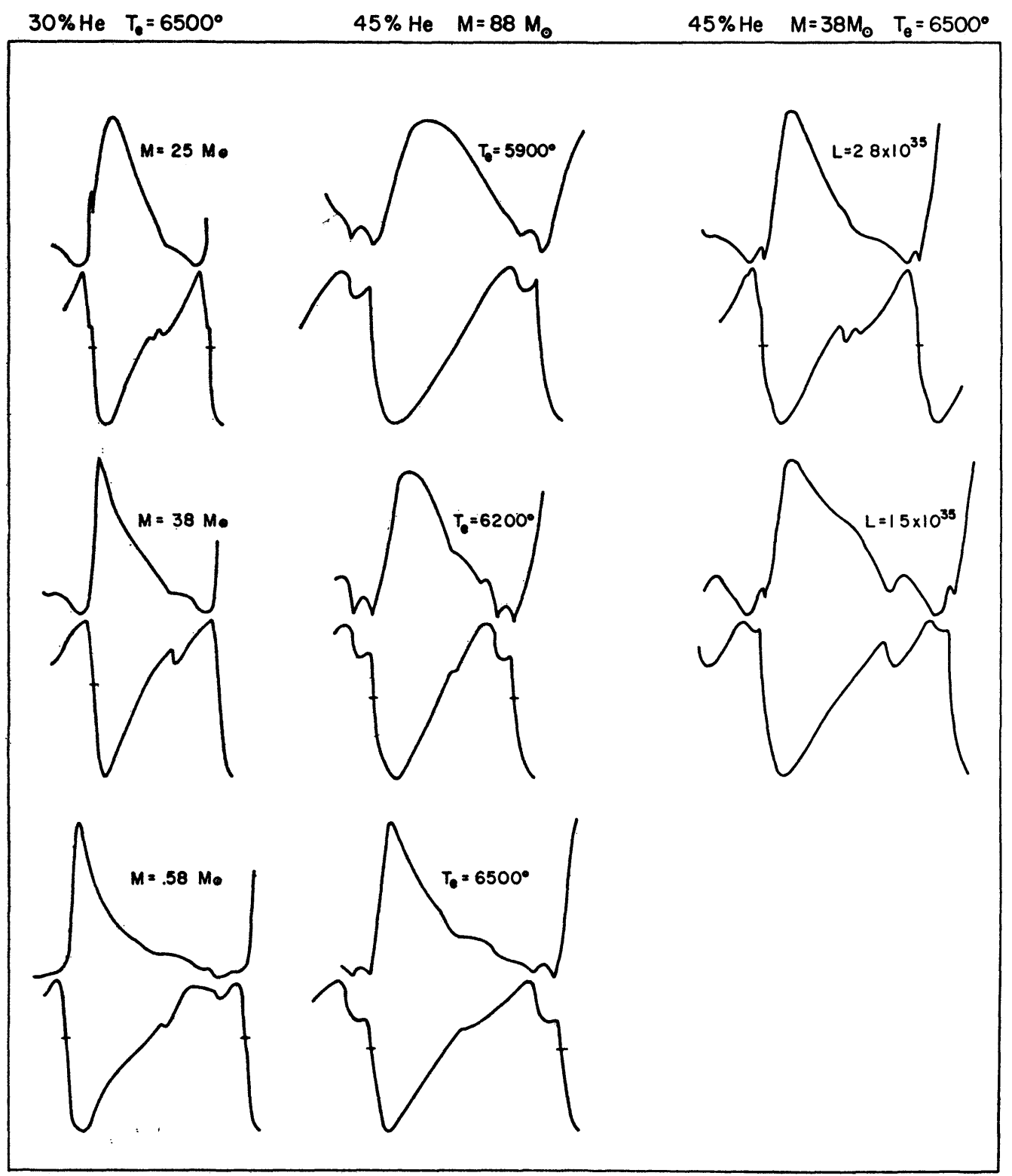

Fig. 32. - Light- and velocity-curves for the fundamental mode for various masses, helium abundances, and luminosities. 
of a general trend. This calculated phase lead of the luminosity rise for type $c$ variables is confirmed by observations (Preston and Paczyński 1964).

It is possible, from the above discussion, that fundamental pulsators of particularly low mass for the luminosity, where we have found that the first harmonic does not become unstable for high $T_{e}$, may at the high- $T_{e}$ side of the instability strip show a rise in luminosity preceding maximum compression. In fact, a few model pulsators with this property were found-particularly $7 \mathrm{~d} F$ and $7 \mathrm{e} \mathrm{F}$. In these cases, however, the rise did not reach such a high peak as in the first-harmonic models. In $7 \mathrm{~d} F$, the rise reached about $\frac{1}{2}$ of the peak luminosity, and the general impression was similar to the well-known "hump" on the rising branch of a fundamental light-curve (Preston and Paczyński 1964).

In model $7 \mathrm{e} F$ the rise in luminosity also occurred early but it reached a peak well below mean luminosity and then dipped to near minimum before the normal rise in luminosity took place. I am indebted to Oosterhoff for calling my attention to a 1.66-day

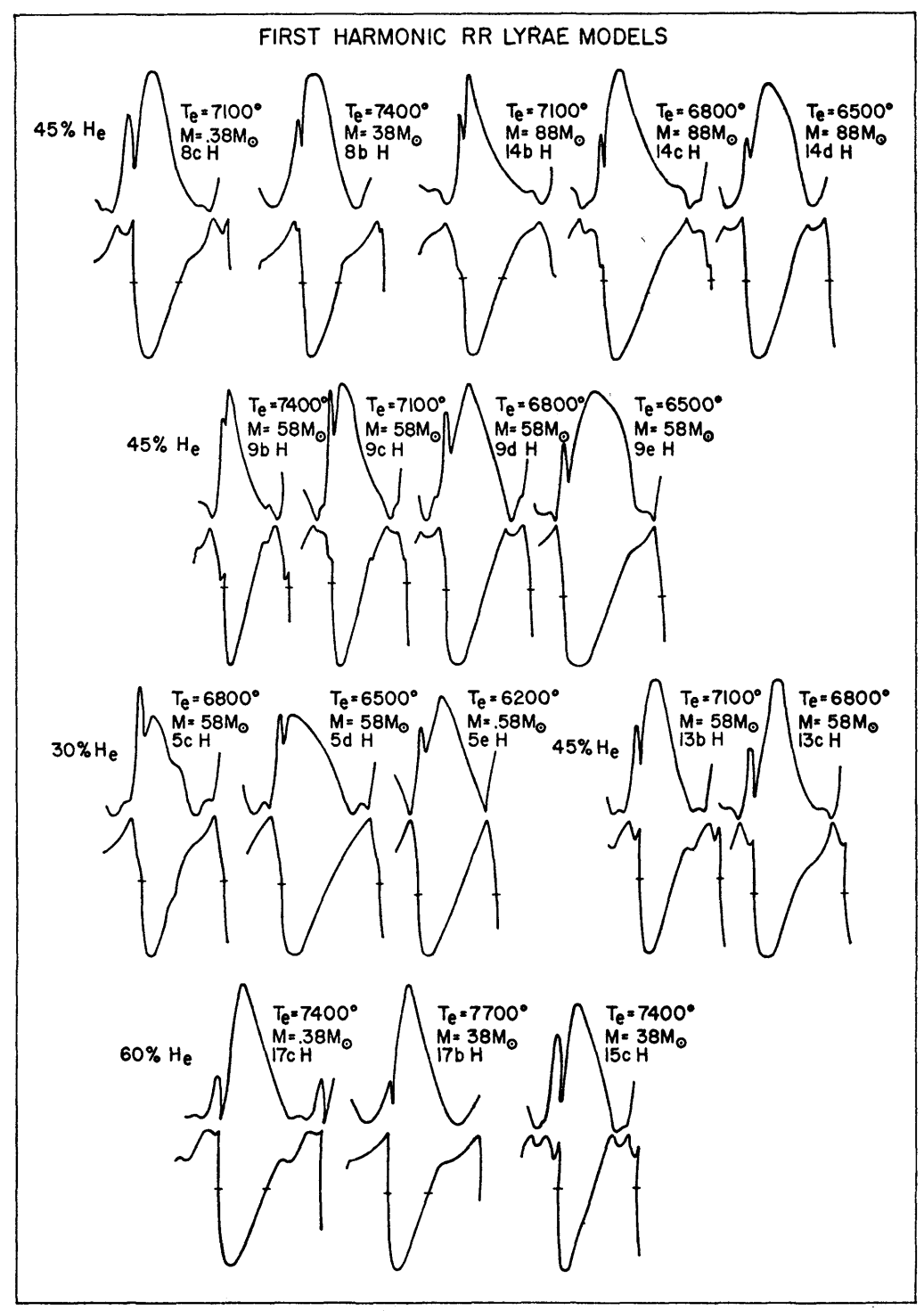

FIG. 33.-Light- and velocity-curves for first-harmonic models 
variable, VZ Aql (Oosterhoff 1943), which shows this behavior. This is presumably the same kind of phenomenon but in a longer-period, more massive, and higher-luminosity variable. It seems that these early rises of the light-curve all have the same basic explanation-it is the normal rise in luminosity resulting from an only slightly delayed flux from the deeper subphotosphere.

In the discussion above the rise in luminosity is considered the normal thing; it is the depression that follows the rise that is special and needs explanation. This differs from

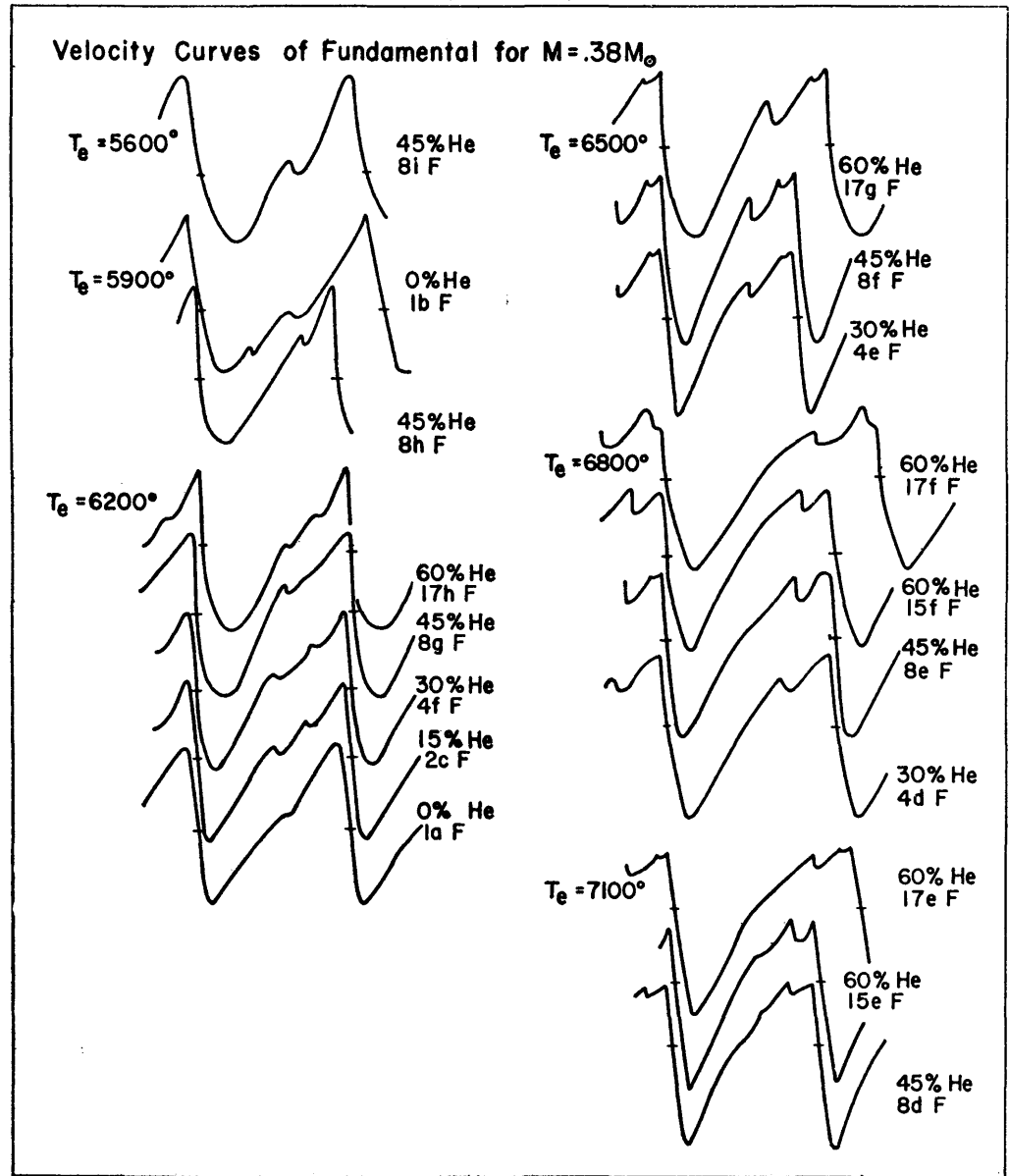

Fig. 34.-Velocity-curves for $M=0.38 M \odot$ and various $T_{0}$ and helium abundances. Scales arbitrary.

the point of view of Tifft (1964), who views the rise as resulting from the transformation of kinetic energy of infalling gas into light in a photospheric collision with the rising gas of the new cycle. In the calculated models, such is not the case. At the time of the first rise in luminosity, only in the region of $\mathrm{He}$ II ionization is the velocity beginning to reverse and the velocity has not begun to reverse in the region of hydrogen ionization until the luminosity is nearing its first peak. The velocity reverses in the photosphere only at the time of the peak.

The explanation given here of the "hump" on the rising branch of a type $a$ variable is quite consistent with that of Oke, Giver, and Searle (1962), who point out that the peak in $U$ at this time is due to the decrease in the Balmer discontinuity associated with large pressures and accelerations in the photosphere. We have noted the same timing, but since 
we are dealing only with the bolometric luminosity we cannot identify a $U-B$ peak in our calculations.

In examining the cause of the dip in luminosity, we noted that in the regions of helium and hydrogen ionization a rise in opacity accompanies compression (see Fig. 28). When the amplitude becomes large, the peak compression appears rather suddenly at the time of velocity reversal, and it appeared that this accompanying rather sharp peak in opacity in the regions of helium and hydrogen ionization was responsible for the dip in luminosity. In this picture the dip in luminosity is associated with the time of maximum compression and with the time of velocity reversal in the photosphere and is due to a temporary increase in opacity in the subphotosphere. We note that the data of Preston and Paczyński (1964) support this view. The dip in luminosity of T Sex occurs between phases 0 and 0.1 which is just the time of velocity reversal.

The same picture may well account for the depression in the rising branch of the

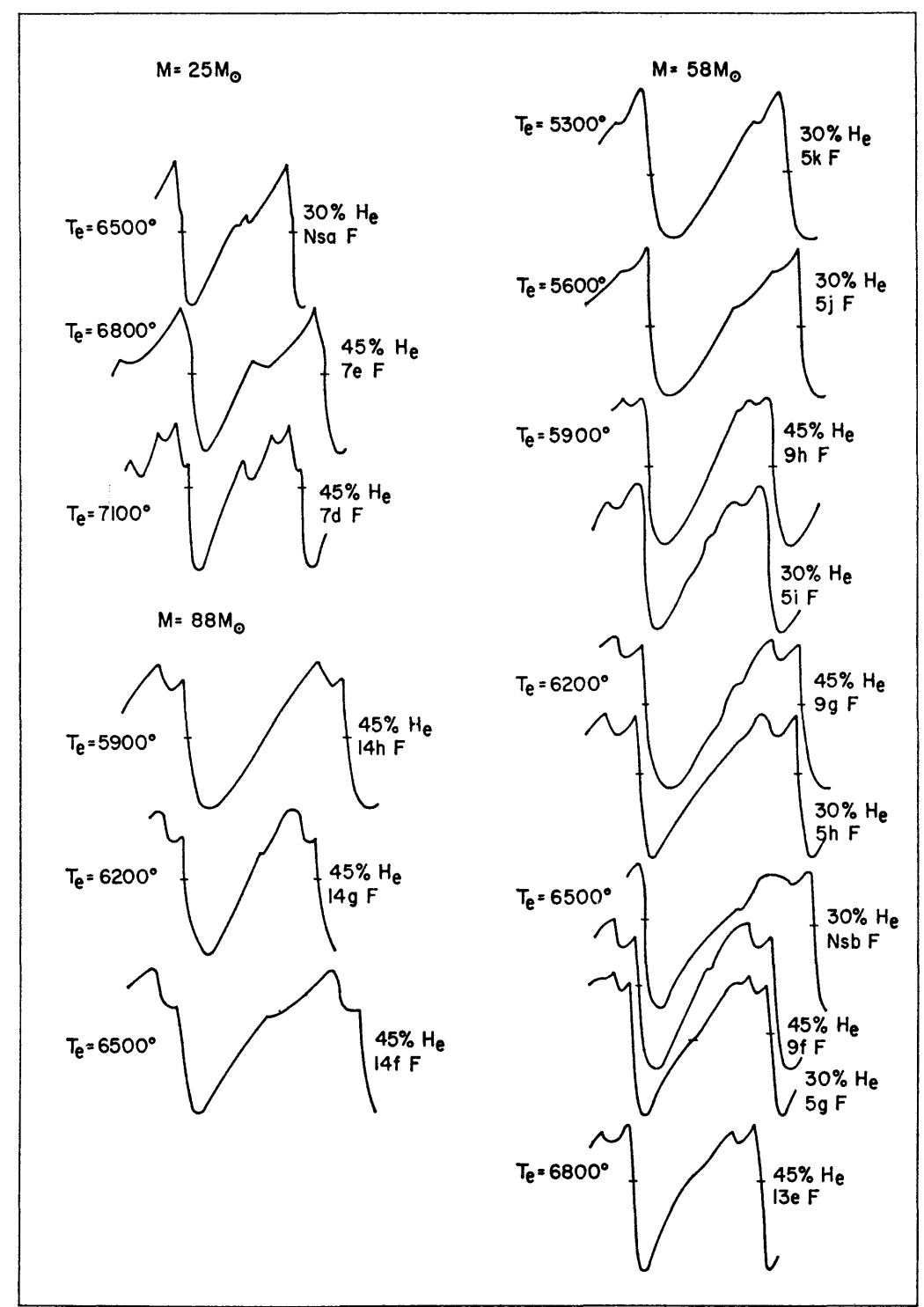

FIG. 35.-Velocity-curves for various masses and $T_{0}$ values 
luminosity-curve of type $a$ variables which makes the "hump" referred to earlier. The work of Preston and Paczyński again is reasonably consistent with this picture as the phase of velocity reversal seems to be correlated with the phase of the depression in luminosity. This feature is rarely very clear in the models we have calculated, which may readily be understood by the fact that it is observationally small and our techniques of calculation of the luminous flux (see Paper II) are somewhat crude.

It should be pointed out that the features we have been discussing are quite non-linear. They appear in the models only for relatively large amplitude. We ascribe this to the fact that the phase relation of luminosity and velocity is quite amplitude-sensitive and the first rise in luminosity occurs significantly earlier and is more prominent near maximum amplitude than at $\frac{1}{2}$ maximum.

The observations of Preston and Paczyński on type $a$ variables, referred to above, clearly shows that the rise in luminosity for type $a$ variables tends to occur slightly earlier than the velocity reversal. Mean luminosity may occur from 0 to 0.01 period prior to zero velocity. Our best model calculations (series 4 and 5) give instead a lag of 0 to $\mathbf{0 . 0 2}$ for the bluer type $a$ variables. It is probable that this indicates a systematic error in the technique as used with only a relatively small number of mass zones. The radiative boundary condition (see Paper II) will itself result in a slight delay in the luminosity, as in our models it is the surface temperature that controls the luminosity rather than the photospheric temperature.

\section{b) Secondary "Bumps" on the Light-Curve}

A persistent feature of the calculations reported here is the appearance during decreasing light of a significant bump or bumps on the light-curve associated with small shocks in the velocity-curve (see Figs. 31 and 32). The small shock or bump in the velocity-curve is particularly noticeable and usually is found between 0.5 and 0.8 of a period after minimum radius. Sometimes there are two small bumps rather than a single large one. The light-curve shows an associated bump corresponding to a temporary heating of the photosphere resulting from the outgoing compressional wave seen in the velocity-curve. The temperature and luminosity show a minimum at the time of the jump in the velocity-curve followed by a small peak about a tenth of a period later.

Such bumps on the light-curve are also known from observation both for RR Lyrae variables and Cepheids (see, e.g., the bump about phase 0.6 for SU Dra [Oke et al. 1962]). The bump on the velocity-curve has not been reported before, but the velocity-curve for SU Dra was drawn (Oke et al. 1962) with a discontinuity in slope.near phase 0.4. We have noted, however, that the data would be consistent with a bump in the velocitycurve similar to those calculated at phase 0.6. We believe, therefore, that SU Dra shows evidence for time-associated bumps on the light- and velocity-curves of the same kind as those appearing in the calculations. A rough examination of unpublished ${ }^{3}$ light- and velocity-curves for $\mathrm{X}$ Arietes and SW Andromeda suggests that these associated features of light-and velocity-curves are a normal characteristic of the dynamics of variable stars. As these features appear to be true results of the calculation and correspond to what is found in nature, we have attempted to learn more about their cause and to find how they correlate with the various parameters that determine the models. In this attempt we have been only partially successful.

The systematics of these features are presented best in the velocity-curves in Figures 34 and 35 . Since our velocity-curves are more precisely and reliably calculated than the light-curves, we could identify these features more consistently in the velocity-curves. Figure 34 shows the considerable regularity in appearance for fixed mass $(0.38 M \odot)$ and a variety of $T_{e}$. For a given $T_{e}$, the curves for differing helium content are quite similar, but the curves progressively change in appearance with $T_{e}$. As $T_{e}$ is raised, the bump be-

${ }^{3} \mathrm{I}$ am indebted to J. B. Oke for many discussions on RR Lyrae stars and for permission to examine unpublished data. 
comes more prominent, reaching a maximum at $6500^{\circ}$ and then remaining prominent but moving toward a later phase as $T_{e}$ is raised further. If we now examine Figure 35, we see in addition a progressive change in character with mass. For $0.25 M \odot$, the bump occurs quite early (near phase 0.5 ). At $0.38 M \odot$, the bump was at about phase 0.65 . For $0.58 M \odot$, the bump is near phase 0.75 and for $0.88 M \odot$ the bump is very late, appearing only as a shoulder just before velocity reversal at about phase 0.85 . Since the velocitycurve feature slightly precedes the light-curve feature (Figs. 31, 32), we are led from the above correlation of phase and mass to identify the mass of observed RR Lyrae stars as about $0.5 M \odot$ since it is in this range of mass that our calculated bumps are most similar to observed ones. It is also possible to use the systematic change of shape of the velocitycurve with $T_{e}$ as a tool for studying observed variables. Actually the comparisons we have made were all with variables of $T_{e}$ near $6500^{\circ}$ where the observed and calculated features are in reasonable correspondence. We have therefore not yet identified in the observations the trend with $T_{e}$ shown in Figure 34 .

An examination of Figure 1 (see above) shows the prominent temperature bump near $t=1630$ (phase $\approx 0.65$ ) at various depths in the model $(4 \mathrm{e} F)$. It seems to originate or amplify strongly at zone 30 near $20000^{\circ}$. It can be traced inward for a few zones where it is lost in a curve which has persistent high harmonic structure superimposed on the fundamental (see zone 19 or 20). In order to understand the feature better the velocities of the interior points were examined in a model with a well-developed surface bump. It was found that the surface feature in the velocity-curve could be traced inward for about 10 mass zones to a region in the helium zone where it became invisible. In the middle mass zones (15-25), the motion was more symmetrical than at the surface but not really sinusoidal. In the interior 5-10 mass zones, the velocity-curve again was found to depart widely from sinusoidal, showing during about one-half of the cycle a very large amplitude of a wave of about one-fifth of the fundamental period. Since this wave was strong in only about one-half period, only about two oscillations of this high harmonic were seen each period. ${ }^{4}$

Although this very strange behavior raises questions about the validity of the calculations, such tests as I have made seem to confirm the correctness of the computed result. The calculated results show a small anomaly in the surface region that becomes progressively weaker in the intermediate region and again amplifies strongly in the inner zones which lie inside 0.5 of the stellar radius. This behavior apparently is related to the behavior of high harmonic modes in linear calculations. Calculations show that high harmonics such as the fifth or sixth have a very small amplitude in the intermediate zones (radii between 0.6 and 0.8 ) when compared to the fundamental at the surface, but amplify very strongly compared to the fundamental in the region inside 0.5 of the radius. Thus the linear calculations show that, if high harmonics are excited in the motion, they will be particularly prominent compared to the fundamental in the inner zones. Our picture of these pecular features, then, is that the very strong pressure peak associated with minimum radius provides a periodic driving force for high harmonics. Certain harmonics can apparently be strongly driven (perhaps by near resonance with the fundamental) and appear markedly in the motions of the interior points and again are noticeable in the motions of the surface zones, where they account for the bumps we are discussing. This explanation is consistent with such checks as we have been able to make: thus if the motion is started smoothly, the anomalies first appear after the required time for a pressure pulse near the surface to communicate to the center and reach the point in question. The fact that near resonance may be needed may help explain why the phase and size of the anomaly in any particular case have not been predictable. We hope that further study of these features which are so characteristic of pulsating stars will permit their unambiguous use as indicators of physical parameters of the star.

4 I am indebted to J. Castor for calling to my attention this strange behavior of the interior points in my calculations. He has also assisted me by discussions on these points and by calculations on harmonic behavior in the linear case. 


\section{c) Fitting a Model to an Observed Variable-SU Draconis}

J. B. Oke and co-workers have made detailed studies of the atmospheres of a few RR Lyrae variables. In particular, we have chosen to relate the observations of Oke et al. (1962) on SU Dra to our calculated models. This follows in general the procedure reported by Christy (1963b) but is more precise because of the large number of fullamplitude models now available.

We prefer to use relative amplitudes rather than absolute amplitudes in such a fitting procedure since we have shown that certain relative amplitudes (such as $\mathbb{R}$, defined earlier) and even the luminosity moment and phase relations are much less sensitive and are more precisely calculated than the absolute amplitude.

From the observational data we find a mean $T_{e} \approx 6425^{\circ}$. The period is 0.660 day, and the mean luminosity occurs at phase 0.90 which is 0.01 earlier than the time of the mean velocity (the lag is -0.01 ). The luminosity was taken to be proportional to $R^{2} / \theta_{e}{ }^{4}$. The luminosity amplitude was $0.95 \mathrm{mag}$. The integrated luminosity amplitude is ILA = 0.118 and the luminosity moment is 0.14 .

Although the evaluation of the luminosity requires considerable precise data or some slight approximation as above, because of the nature of observational techniques it can be done rather accurately. The evaluation of the velocity and radius variations is, on the other hand, subject to considerable random as well as systematic error. The velocitycurve itself refers to a variable mass layer in the atmosphere. We have seen that the bulk of the atmosphere (excluding the very high layers where the hydrogen lines are formed as our calculations were not accurate for such thin layers) shows no appreciable velocity gradient from the time of radius maximum to minimum. There is, however, some gradient during the time when the radius is increasing. To allow for this gradient, Oke et al. (1962) have chosen to redraw the corresponding portion of the observed velocitycurve to eliminate the observed kink at phase 0.4 . This led them to the $\gamma$-velocity of $-161.0 \mathrm{~km} / \mathrm{sec}$ and to a value of $\Delta R$ from integrating the velocity-curve from maximum radius to minimum. We have seen in our models (Figs. 34 and 35) that features related to the "kink" in the velocity-curve are a standard feature of the model calculations and therefore should not be eliminated from the observations. Although we are aware that there should be a small correction to the observations in the direction of Oke's correction, we do not now have a good value for it and we believe that it is negligible. That is, we will interpret the observations as though they pertained to a single layer.

In this way, we deduced a $\gamma$-velocity of $-166.5 \mathrm{~km} / \mathrm{sec}$ (a slight overestimate). We then integrated a relative radius-curve from the velocity (after multiplying by the usual correction $\frac{24}{17}$ to correct from projected velocity to radial velocity with respect to the star). We are not satisfied that this correction factor is reliable, but it will require a dynamic atmosphere calculation to revise it. This radius-curve gave $\Delta R=6.76 \times 10^{10}$ $\mathrm{cm}$ compared to Oke's $3.5 \times 10^{10} \mathrm{~cm}$. The radius-curve calculated from the velocitycurve was then fitted to Oke's photometric results for $R / R_{\max }$. The curve fitted reasonably well over all phases (maximum radius was at phase 0.35 ) in view of the scatter of the points, and we found $\Delta R / R=0.137$, and $R_{\text {mean }}=4.93 \times 10^{11} \mathrm{~cm}$. In addition to the uncertainty in interpreting the velocity-curve to which we have already referred, we found also considerable uncertainty (about 15 per cent) in fitting the relative radius variation. We conclude that the resulting value for $R_{\text {mean }}$ may be uncertain by 30 per cent. Thus, Oke's result $R_{\text {mean }}=3.60 \times 10^{11} \mathrm{~cm}$ would not be excluded by our fitting procedure.

The value for $R$ that results is $0.137 / 0.118=1.16 \pm 0.15$. We can now compare these observatiional results with the model quantities. We see that a model with properties near those of $14 \mathrm{f} F$ would be a good fit. The value of $Q \approx 0.0360$ then would lead to a value $\rho / \rho \odot=0.00297$ and, with the value of $R_{\text {mean }}$ above, we get $M=2.1 \times 10^{33}$ gm. Associated with this radius and $T_{e}=6425^{\circ}$, we get

$$
L_{0}=2.96 \times 10^{35} \mathrm{ergs} / \mathrm{sec} \quad \text { or } \quad M_{\mathrm{bol}}=+0.02 \text {. }
$$


However, the uncertainty in these values is quite large, arising primarily from the large uncertainty in the radius. For example, the radius $3.60 \times 10^{11} \mathrm{~cm}$ found by Oke would fit better with model $4 \mathrm{e} \mathrm{F}$ (although the observed $\Delta R / R$ is smaller than in the model). Then $Q \approx 0.0385$ which leads to a result $M=0.935 \times 10^{33} \mathrm{gm}$. Associated with this radius and $T_{e}=6425^{\circ}$, we get

$$
L_{0}=1.57 \times 10^{35} \mathrm{ergs} / \mathrm{sec} \quad \text { or } \quad M_{\mathrm{bol}}=+0.71 .
$$

These two results are indicative of the very considerable uncertainty in mass that follows from the use of the mean radius deduced from observation. No doubt more study of motions in the atmosphere and more detailed comparison with dynamic model atmospheres could remove a considerable part of this uncertainty.

Two other methods of mass determination are available. One involves the use of a luminosity to determine the radius and the other involves the use of results on the shapes of velocity-curves discussed previously.

The determination of the luminosity from the shortest fundamental period in clusters (see below) leads to $L_{0} \approx 1.50 \times 10^{35} \mathrm{ergs} / \mathrm{sec}$. With this luminosity and $T_{e}=6425^{\circ}$, and the period 0.660 day, we find (see Table 2$) Q \approx 0.0385$ from which $R=3.51 \times 10^{11}$ $\mathrm{cm}$ and $M=0.87 \times 10^{33} \mathrm{gm}$.

The above discussion shows that we have difficulty in determining the mass to within a factor of 2 from the use of the modified Wesselink method. If the luminosity can be determined to better than a factor of 2 , the radius can be found from the temperature and the mass from the period and $Q$. In what follows, we will assume $L_{0}=1.50 \times 10^{35}$ ergs/sec $\left(M_{\mathrm{bol}}=+0.76\right)$, as later arguments and the previous mass determination point at this value and our model sequences are most complete at this value.

The first step in comparing SU Dra with the models is to decide which of the different helium abundances fits best the observations. We have seen that the luminosity moment, $L M$, and the amplitude ratio $R$ correlate well with each other and each varies consistently with $T_{e}$ and with helium content. Thus, from $T_{e}=6425^{\circ}$ and $R=1.16 \pm 0.15$, we deduce (see Fig. 10) the helium content $31 \pm 6$ per cent. The value $L M=0.14$ corresponds (Fig. 11) to a helium content of 29 per cent; an uncertainty of 0.01 in $L M$ would imply an uncertainty of 4 per cent in helium content. Similarly, we find that an uncertainty of 1 per cent in $T_{e}$ corresponds to 3 per cent uncertainty in helium content. These results show that the quantities $R$ and $L M$ are consistent with each other and are indeed sensitive measures of the location of a given variable in the instability region. If $T_{e}$ of the variable is known, this then determines the location of the instability region in terms of the range of $T_{e}$ which in turn is sensitive to the helium content of the atmosphere. In particular, $L M$ can be determined from luminosity data alone and, if systematically studied, can well add a new measure to the study of variable stars.

Having determined that the data of SU Dra fits well the calculations for 30 per cent helium if $L_{0}=1.50 \times 10^{35} \mathrm{ergs} / \mathrm{sec}$, we can now compare other observed and calculated quantities. The phase lag predicted by the models is about +0.02 period whereas it is observed to be -0.01 . This difference may well represent a systematic error in the calculation of the diffusion approximation in the luminous flux in the subphotosphere. The mass resulting from the assumption $L_{0}=1.50 \times 10^{35} \mathrm{ergs} / \mathrm{sec}$ is $M=0.875 \times 10^{33}$ gm which lies between sequences 4 and 5 . The radius is $3.50 \times 10^{11} \mathrm{~cm}$.

The principal discrepancies between the models and observation appear to lie in the amplitudes of the motion. The observed luminosity amplitude of $0.95 \mathrm{mag}$. is in reasonable agreement with what would be expected from sequence 14 with $L_{0}=2.80 \times 10^{35}$ ergs/sec but is significantly smaller than is calculated for sequences 4 and 5 with $L_{0}=$ $1.50 \times 10^{35} \mathrm{ergs} / \mathrm{sec}$. In trying to reconcile the observations with calculations in sequences 4 and 5 , we recognize that the actual amplitude of the computed motion is probably the aspect of the calculations that is most subject to correction. If the work generated per period has been somewhat overestimated, the calculated amplitude would be too great and the discrepancy would be eliminated. 
In the same way that the calculated luminosity amplitude is too great, so is also the calculated radius amplitude $\Delta R / R$ for sequences 5 and 4 . Sequence 14 , however, shows good agreement with observation also for $\Delta R / R$.

Other observational data on RR Lyrae stars can similarly be compared with computed models. The models serve as a basis of comparison and also as a test of consistency. When many observed stars have similarly been compared with calculations, it should become possible to appraise better the agreement or disagreement with observation.

\section{d) The Periods of Cluster and Field RR Lyrae Variables}

Preston (1959) has made a spectroscopic study of RR Lyrae stars and has shown a correlation of metal abundances (as determined by the difference of the hydrogen and $\mathrm{K}$-line spectral types) with the period. He shows that the strong-line variables as a group have shorter mean periods than the weak-line group, and he points out that Oosterhoff's type I clusters have variables with mean type $a$ periods similar to the weak-line group, whereas the Oosterhoff type II clusters have longer mean periods for the type $a$ variables. Deutsch and also Kinman (1959), among others, have shown that the metal abundance of the type II clusters is characteristically less (very weak lines) than the type I clusters (weak lines).

Sandage and Wallerstein (1960) classify clusters according to the difference in magnitude $\Delta V$ between the tip of the red-giant branch and the horizontal branch. They note that this difference is greatest in the clusters with very weak metal lines and the longest periods. The difference is smallest in the clusters with strong metal lines. In these latter clusters the horizontal branch is populated only at the red end, whereas in the clusters with very weak metals it is populated only at the blue end. Clusters with weak metals lie between the above extremes and have periods shorter than the very weak line clusters. Sandage (1958) has suggested that if the RR Lyrae stars of different mean periods are assumed to have the same mass and colors, then the period difference implies a radius and a luminosity difference such that the longer periods are associated with greater luminosity according to $L \propto R^{2} \propto P^{4 / 3}$.

We have found a new type of relation between period and luminosity: the shortestperiod fundamental mode (type $a$ ) or longest-period first harmonic (type $c$ ) in a group of definite luminosity spanning the instability strip varies approximately as $P \sim L^{06}$, and this relation is independent of variations in mass and composition. The calibration of this law resulting from these calculations is

$$
P_{\mathrm{tr}}=0.057(L / L \odot)^{0.6} \quad \text { 'or } \quad L / L_{\odot}=\left(\frac{P_{\mathrm{tr}}(\mathrm{days})}{0.057}\right)^{1.67}
$$

By applying this relation to the clusters and to data on field variables, we are thus able to deduce different luminosities for the RR Lyrae stars in such a way that the groups with the shortest periods, which are associated with the strongest metal lines, have the lowest luminosities and the groups with the longest periods, associated with the weakest metal lines, have highest luminosities. We have examined the period collections of a few clusters and show in Figures 36 and 37 the period distributions of M3 (Hogg 1955) and $\omega$ Cen (Martin 1938). There are usually a few stars with periods in the gap between the main group or type $a$ periods and that of the type $c$ periods; these may correspond to the situation discussed earlier where both instabilities are possible depending on the history of the star. A type $a$ variable in the gap would then be thought to be evolving to the left and a type $c$ to the right in the H-R diagram. By setting the ratio of shortest type $a$ to longest type $c$ periods equal to $\frac{4}{3}$, and fitting the distribution of periods as observed, we have arrived at values for the transition period $\dot{P}_{\mathrm{tr}}$ for the clusters listed in Table 4 .

The list still does not include the population with relatively normal metal content. Preston's compilation and that of Kinman, Wirtanen, and Janes (1965) make it clear 
that the type $a$ periods of this group extend to shorter values than 0.45 day, but the absence of a clear group of $c$ 's to compare to the $a$ 's and the difficulties of selection have not permitted a firm choice of $P_{\mathrm{tr}}$ among these variables. If $P_{\mathrm{tr}} \approx 0.43$ day, then we would deduce $L \approx 1.13 \times 10^{35} \mathrm{ergs} / \mathrm{sec}$ or $M_{\mathrm{bol}}=+1.06$.

The relative numbers of type $a$ and type $c$ variables in a cluster or the shape of the period frequency plots can also reveal additional properties of these stars. The histogram of Figure 37 for $\omega$ Cen shows about the same frequency per unit $\log P$ of type $c$ as of

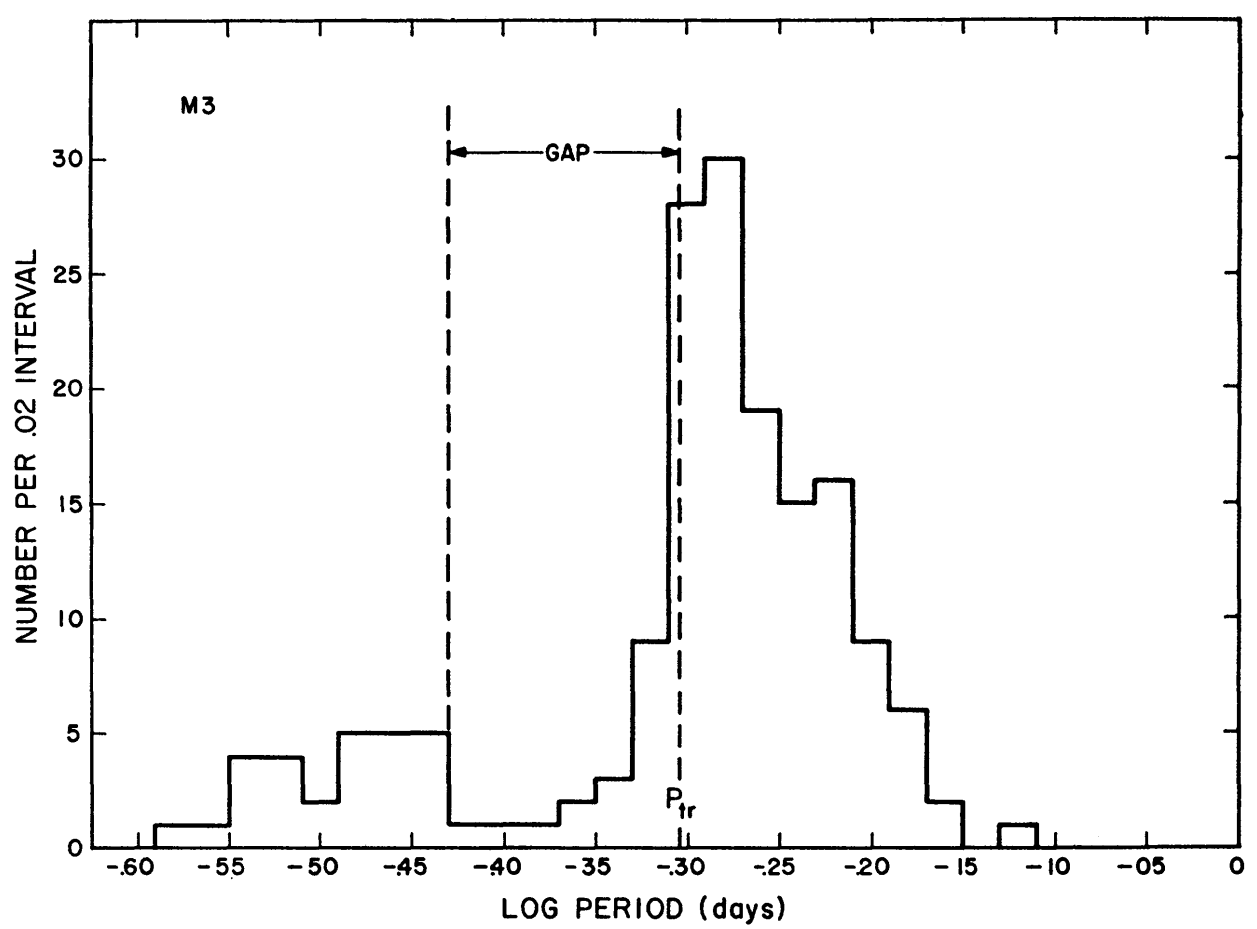

FIG. 36.-The frequency distribution of the periods of variable stars in M3 showing the gap marking the transition from fundamental to first harmonic.

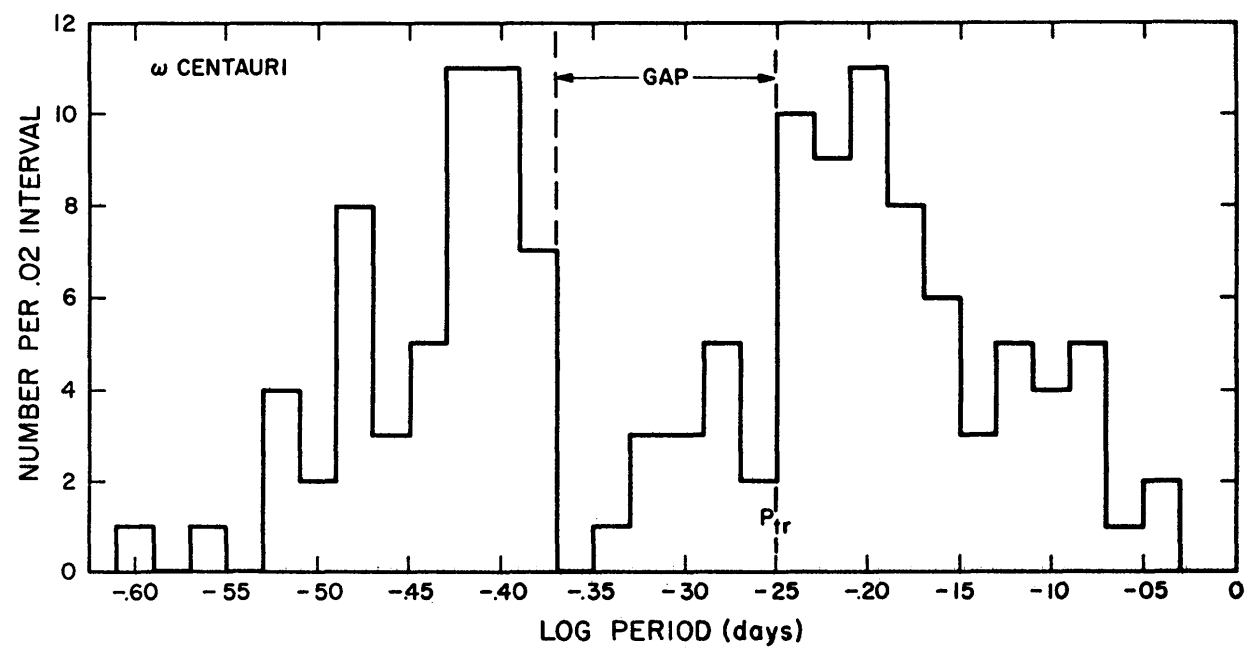

Fig. 37. - The frequency distribution of the periods of variable stars in $\omega$ Centauri showing the gap marking the transition from fundamental to first harmonic. 
type $a$ variables. This is what would be expected if variables evolved either from $a$ to $c$ or $c$ to $a$ or indeed if a roughly uniform population in color evolved vertically with a pause at the horizontal branch. On the other hand, in M3 (Fig. 36), the distribution is completely different. From long to short periods, we see that the frequency rises to a peak near 0.5 days and then after jumping to the $c$ type, the frequency is very much reduced (to $\frac{1}{4}$ or less). We can only conclude that only a few of the stars when evolving in either direction in the variable region are able to become first-harmonic pulsators; 80 per cent of the stars in the variable region are able to pulsate only in the fundamental. These numbers could be modified somewhat if evolution proceeded considerably more rapidly in the type $c$ region, but the discontinuity in frequency at just the transition from $a$ to $c$ can hardly be an accident. We believe it must be real and proceed to examine how to understand this result.

An examination of Table 2 and Figures $2-7$ shows that for a definite helium abundance of 30 per cent, $M=0.75 \times 10^{33} \mathrm{gm}$ does not show a first harmonic instability whereas $M=1.15 \times 10^{33} \mathrm{gm}$ shows a well-developed first-harmonic sequence. A mass somewhat greater than $0.75 \times 10^{33} \mathrm{gm}$ would lead to a minimum type $a$ period about 0.5 days and still would not give a first-harmonic instability. Thus we could explain the M3 frequency

TABLE 4

Transition PeRIods aNd Luminosities OF Clusters

\begin{tabular}{|c|c|c|c|c|}
\hline Cluster & $P_{\text {tr }}$ (days) & $\begin{array}{c}L \text { (calc.) } \\
\left(10^{35} \mathrm{ergs} / \mathrm{sec}\right)\end{array}$ & $M_{\text {bol }}$ (calc) & Metals (Deutsch) \\
\hline $\begin{array}{l}\omega \text { Cen } \\
\text { M15 } \\
\text { M3 . } \\
\text { M5 } . . .\end{array}$ & $\begin{array}{r}0565 \\
.565 \\
496 \\
0455\end{array}$ & $\begin{array}{ll}1 & 78 \\
1 & 78 \\
1 & 44 \\
1 & 24\end{array}$ & $\begin{array}{r}057 \\
.57 \\
80 \\
0 \quad 96\end{array}$ & $\begin{array}{l}\mathrm{B}\left\{\begin{array}{l}(\text { weak }) \\
(\text { Kinman })\end{array}\right. \\
\mathrm{C} \text { (very weak) } \\
\mathrm{AB} \text { (weak) } \\
\mathrm{A} \text { (slightly weak) }\end{array}$ \\
\hline
\end{tabular}

distribution if the horizontal-branch stars in M3 have 30 per cent helium and a range of masses from about 0.45 to $0.60 M \odot$. The mean $T_{e}$ at the transition period would be in the range $6500^{\circ}-6800^{\circ}$. If we attempt to fit with 45 per cent helium (see Fig. 4), we would require the mass to be in the range $0.3-0.45 M \odot$ and the mean $T_{e}$ at the transition would be in the range $6800^{\circ}-7200^{\circ}$. If we fit with 15 per cent helium (see Fig. 2), we would need a mass of perhaps $0.55-0.8 M \odot$. These masses are, of course, all predicated on $L_{0}=1.50 \times 10^{35} \mathrm{ergs} / \mathrm{sec}$ which gives $P_{\mathrm{tr}} \approx 0.5$ day as observed in M3.

Alternatively, we could account for the discontinuity in frequency by means of a definite mass and a range of helium abundances. Thus, from Figures $2-4$ we see that a mass of $0.5 M \odot$ and helium abundances ranging from 15 to 45 per cent would span the observed periods and give the correct transition period. It would require a preponderance at 15 per cent helium to correspond to the frequency discontinuity.

To distinguish these alternatives and to establish in addition the helium abundance in these envelopes, we need data on the values of $T_{e}$. We note that, if the mass is fixed, the transition from $a$ to $c$ takes place at a definite $T_{e}$ independent of helium abundance but the high- $T_{e}$ boundary varies from about $6600^{\circ}$ for 15 per cent helium to about $7500^{\circ}$ for 45 per cent helium which can probably be excluded by the observed sharp color boundary of the variable region. On the other hand, if the helium abundance is fixed at, say, 30 per cent, the high- $T_{e}$ boundary of the type $c$ group will be almost independent of mass at about $7100^{\circ}$ but the transition from $a$ to $c$ will depend on mass somewhat. We would predict that the shortest-period type $a$ variables of low mass would persist to about a maximum $T_{e} \approx 6900^{\circ}$ and would be stable at higher $T_{e}$, whereas the higher-mass type $a$ variables would become $c$ 's at as low as $6500^{\circ}$ and persist as $c$ 's to 
about $7100^{\circ}$. In view of the difficulty in establishing observationally the mean $T_{e}$ of a large-amplitude variable, these differences may not be measurable for the type $a$ group, but a range of $T_{e}$ associated with a given period may be determinable for the smallamplitude type $c$. Roberts and Sandage (1955) in fact show some width to the colorperiod relation that would be consistent with the above views, but it is not certain that their mean colors were determined with sufficient precision to prove the existence of a range of masses. ${ }^{5}$ Our estimate of a mass range of 4 to 3 would, for fixed luminosity, imply a period range of 15 per cent at fixed color or a range of about 0.07 in mean color index at fixed period.

Note added in proof.-Recent observations on $\omega$ Centauri by Dickens and Saunders (1965) and by E. Geyer (private communication) show an overlap in color of the type $c$ and type $a$ variables and of the blue horizontal-branch stars with the type $c$ variables which is discussed above in connection with M3. Observations by A. Sandage (private communication) indicate a similar overlap in color of the type $c$ and $a$ variables in M15 but do not show an overlap of non-variable blue stars with the type $c$. This suggests that a range of masses is also present in these clusters. It is surprising that, whereas it was the small ratio of $c$ 's to $a$ 's in M3 that led to a prediction of a range in masses and an overlap in color at the $c, a$ boundary, this phenomenon is found in $\omega$ Cen and M15 but has not been seen in M3 by Roberts and Sandage (1955).

We can now draw some conclusions about the helium abundance in the envelopes of the $\mathrm{M} 3$ variables provided we use the mass determination of $\approx 0.5 M \odot$ resulting from the discussion of the shapes of light- and velocity-curves. We saw that the phase of secondary bumps pointed to masses in the range $0.35-0.55 M \odot$. If we use this mass estimate together with the luminosity $L_{0}=1.50 \times 10^{35} \mathrm{ergs} / \mathrm{sec}$, we can fix the helium abundance of M3 in the range $25-40$ per cent (by mass). It is not quite clear what the combined uncertainty of this result is, but we could probably exclude 15 per cent helium on one side and 50 per cent helium on the other. In any case we are definitely led to ascribe a helium abundance which is nearer to conventional population I estimates than population II.

One of the remarkable conclusions following from our determination of luminosities between 1 and $2 \times 10^{35} \mathrm{ergs} / \mathrm{sec}$ for RR Lyrae variables, is the determination of mass values in the neighborhood of $0.5 M \odot$. In order to admit masses of $1.0 M \odot$ for these stars and $T_{e}$ in the observed range, we would require a luminosity of $3.0 \times 10^{35} \mathrm{ergs} / \mathrm{sec}$ or $M_{\text {bol }} \approx 0.0$, and, in addition, our determination of the transition between first harmonic and fundamental would have to be systematically in error. Now, since we have not yet been able to understand what physical conditions determine this transition, and we have neglected convection, we cannot be completely sure that such a systematic error is absent. However, the finding that the variables in M3 appear to encompass a range of 33 per cent in mass would be understandable if they had all lost about half their original mass but would be inexplicable without assuming mass loss. Observations on the distribution of horizontal-branch stars in M3 have already led Woolf (1964) to conclude a loss of at least 20 per cent in mass.

\section{DISCUSSION}

\section{a) Models of Horizontal-Branch Stars}

Only a few published models exist for horizontal-branch stars. Cox and Salpeter (1961) constructed approximate models of helium-core stars with hydrogen-rich envelopes of

5 Dr. A. Sandage has recently informed me that the boundaries of the instability strip for M3 are $B-V=0.17$ and 0.44 , corrected for reddening. Using Oke's (1962) relation between $B-V$ and $T$. which was derived for an RR Lyrae star of low metal content, SU Draconis, these boundaries become $7130^{\circ}$ and $5980^{\circ}$. From Table 1 , we see that the high- $T_{e}$ boundary agrees with that calculated for 30 per cent helium (sequence 5 , which also shows the correct periods). We conclude that the envelopes of the variable stars in M3 behave in the same way as calculated envelopes with 30 per cent helium. 
small fractional mass based on pure helium models of Cox and Giuli (1961). In these models, the luminosity was determined primarily by the mass of the helium core for the low-mass envelopes they employed. Hayashi, Hōshi, and Sugimoto (1961) studied the evolution of a $0.7 M \odot$ star based on a pure helium core of $0.53 M \odot$. Also, Härm and Schwarzschild (1964) have studied the evolution of a $1.3 \mathrm{M} \odot$ star through the helium flash to the horizontal branch. The essential results of these calculations are found in Table 5.

In addition, Hayashi et al. and Härm and Schwarzschild have published some results on the mass of the helium core for various models at the time of the helium flash. They are summarized in Table 6.

TABLE 5

LUMINOSITIES OF HORIZONTAL-BRANCH MODELS

\begin{tabular}{|c|c|c|c|c|c|c|}
\hline \multirow{2}{*}{ Mass $/ M_{\odot}$} & \multirow{2}{*}{$\begin{array}{c}\text { CoRE } \\
\text { MASS } / M_{\odot}\end{array}$} & \multicolumn{3}{|c|}{ Original Composition } & \multirow{2}{*}{$\begin{array}{l}\text { LUMINosity } \\
(\mathrm{erg} / \mathrm{sec})\end{array}$} & \multirow{2}{*}{ REFERENCE } \\
\hline & & $X$ & $\boldsymbol{Y}$ & $Z$ & & \\
\hline $\begin{array}{lll}0 & 5 & \cdots \\
1 & 0 & \cdots \\
0 & 7 & \cdots \\
1 & 3 & \cdots\end{array}$ & $\begin{array}{ll}0 & 5 \\
1 & 0 \\
0 & 53 \\
0 & 60\end{array}$ & $\begin{array}{l}\cdots \\
\dot{0} \\
0\end{array}$ & $\begin{array}{ll}1 & 00 \\
1 & 00 \\
0 & 099 \\
0 & 099\end{array}$ & $\mid \begin{array}{lll}\cdots & \cdots & \cdots \\
0 & 001 \\
0 & 001\end{array}$ & $\begin{array}{ll}1 & 56 \times 10^{35} \\
1 & 24 \times 10^{36} \\
3 & 25 \times 10^{35} \\
4 & 57 \times 10^{35}\end{array}$ & $\begin{array}{l}\text { Cox and Giuli (1961) } \\
\text { Cox and Giuli (1961) } \\
\text { Hayashi et al. (1961) } \\
\text { Härm and Schwarzschild (1964) }\end{array}$ \\
\hline
\end{tabular}

TABLE 6

CORE MASSES

\begin{tabular}{|c|c|c|c|c|c|}
\hline \multirow{2}{*}{ MASs $/ M_{\odot}$} & \multirow{2}{*}{$\begin{array}{c}\text { CoRE } \\
\text { MASS } / M_{\odot}\end{array}$} & \multicolumn{3}{|c|}{ Original Composition } & \multirow{2}{*}{ REFERENCE } \\
\hline & & $X$ & $\boldsymbol{Y}$ & $Z$ & \\
\hline $\begin{array}{lll}1 & 3 & . \\
1 & 3 & . \\
1 & 3 & . \\
1 & 0 . . & \cdots \\
1 & 3 \ldots\end{array}$ & $\begin{array}{r}042 \\
.53 \\
.60 \\
59 \\
056\end{array}$ & $\begin{array}{r}061 \\
90 \\
90 \\
.90 \\
90 \\
090\end{array}$ & $\begin{array}{l}0.37 \\
099 \\
.099 \\
.099 \\
0.09\end{array}$ & $\begin{array}{ll}0 & 02 \\
.001 \\
001 \\
001 \\
0 & 01\end{array}$ & $\begin{array}{l}\text { Hayashi et al. (1961) } \\
\text { Hayashi et al. (1961) } \\
\text { Härm and Schwarzschild (1964) } \\
\text { Härm and Schwarzschild (1964) } \\
\text { Härm and Schwarzschild (1964) }\end{array}$ \\
\hline
\end{tabular}

From these results, it would appear that a helium core of about $0.5 M \odot$ with a lowmass hydrogen-rich envelope of perhaps $0.1 M \odot$ could indeed be a model of a horizontalbranch star with $L \approx 1.5 \times 10^{35} \mathrm{ergs} / \mathrm{sec}$ as we have concluded. Hayashi et al. note that the decrease in core mass in going from their population II mixture to their population I mixture is primarily due to the increase in helium. We conclude that our preferred models with $Y=0.30$ would have core masses smaller than found by Härm and Schwarzschild with $Y=0.099$, and would probably lie in the range $0.4-0.5 M \odot$. In addition, we note that the core mass increases somewhat with decreasing metal abundance. This is consistent with our result that the luminosity is greater for the very low metal-abundance RR Lyrae stars than for the ones with only slightly low metal abundance. Further interior model and evolutionary calculations will be needed in order to check our results on the masses and luminosities of RR Lyrae stars, but existing calculations seem to be quite consistent with them.

Note added in proof.-Suda and Virgopia (1966) have recently published a series of possible horizontal-branch models. Their results are also consistent with the choices of 
luminosity and mass made above for RR Lyrae variables. J. Faulkner $(A p . J$., to be published) has also constructed a set of models which include as possible models those we have chosen.

The evolutionary picture for the stars of mass near $1.0 M \odot$ that results from these considerations is as follows. In the course of hydrogen-burning evolution, the stars develop a helium core, the mass of which ultimately is the most significant factor determining their luminosity in the helium-burning stage. At a certain core development, the helium flash occurs and the star reverses its evolution toward the horizontal branch. Since our results imply masses of only $\sim 0.5 M \odot$, we infer that there is a loss of mass between the red-giant phase and the RR Lyrae phase. This mass loss may accompany dynamic effects of the helium flash or a recently discovered thermal instability associated with a helium-burning shell (Schwarzschild and Härm 1965). Alternatively, the mass loss may be a result of pulsation itself.

Calculations (to be published) on a model of a W Virginis star with mass $\approx M \odot$ and $M_{\text {bol }} \approx-3$, which was constructed to duplicate the observed behavior of $W$ Virginis itself, showed a very violent shock wave associated with the pulsation. During the calculation, the surface layer of mass $20 \mathrm{gm} / \mathrm{cm}^{2}$ was being driven away from the star by the momentum transported in the shock. This clearly indicated the possibility of mass loss from pulsation and showed that the very luminous W Virginis phase of cluster variables is likely to provide the most rapid mass loss. In addition, it is possible that the RR Lyrae pulsation phase is itself a significant mass-loss phase.

As a result of mass loss, the stars then arrive on the horizontal branch with a helium core of $0.4-0.5 M \odot$ and a total mass not much greater. Whether their subsequent evolution is governed by nuclear-burning effects in the interior or by continued mass loss resulting from pulsation and supersonic effects in the atmosphere remains for further study.

\section{b) The Long-Period Modulation}

RR Lyrae itself is well known to exhibit a 41-day cycle of modulation which has been recently studied extensively by Preston; Smak, and Paczynski (1965). There is no hint in any of the calculations in this survey of any such phenomenon. I have referred to a 3-period modulation which arises from a mixture of the fundamental and first harmonic, but no periods with such a small ratio as needed for the long-period modulation have been found.

We have noted, however, that the 41-day period would be a reasonable rotation period. Accordingly we have tentatively adopted the working hypothesis that the 41-day period is in fact the result of the pulsation of a non-spherical star which rotates and presents different aspects of its non-spherical appearance to us in the course of time. As a suggestion of the nature of the non-sphericity we have considered a magnetic field which is not symmetric with respect to the rotation axis.

We have mentioned this problem not because we regard the above discussion as an adequate explanation - that will need much more observation and very extensive analysis -but because we believe that such a picture presents the possibility of explaining the phenomenon. Basically we regard the modulation phenomenon as a superficial manifestation rather than a deep-seated deviation from the kind of theory presented in this paper.

\section{c) Cepheid Variables}

The techniques of this paper can be applied to Cepheid variables as easily as to $R R$ Lyrae variables. A few such calcuations have been carried out-specifically for models of $\delta$ Cephei, $\eta$ Aquilae, and W Virginis. These will be reported separately. The only reason that this survey was confined to RR Lyrae models was to permit sufficiently detailed coverage of the remaining physical parameters to allow adequate exploration of the pulsation phenomenon. The calculations on Cepheid and W Virginis models indi- 
cates that there is no difference in the physical phenomena involved. The differences arise solely from the different masses and luminosities of the underlying star.

One result for Cepheid models is suggested by the luminoity dependence of the transition period between first harmonic and fundamental. The increase in the transition period with luminosity is less rapid than the general increase of Cepheid periods with luminosity. As a result, we find that the transition period appears to cross the Cepheid region in such a way that at the short-period low-luminosity end, the stable pulsation mode should be the first harmonic whereas at the long-period high-luminosity end, the stable pulsation mode should be the fundamental. Our approximate formula for the transition period would suggest that the transition would occur near 7 days. We recall the well-known change that is found in Cepheid characteristics near 9 days period and we are led to propose that the short-period (less than 9 days) group of Cepheids are predominantly first-harmonic pulsators, whereas the long-period group are predominantly fundamental pulsators. This proposal will need extensive confirmation by model calculations, but it appears to be consistent with the Eggen type $\mathrm{C}, \mathrm{AB}$ separation (Eggen 1951).

\section{d) Future Work}

The principal deficiency of the present calculations is the omission of convective heat flow. We have presented arguments that this omission is probably not serious on the high-temperature side of the instability strip but may be dominant on the low-temperature side. A major aim of future computations should be a realistic treatment of convection and its interaction with pulsation. Radiation pressure was also omitted from the equation of state in the calculations reported here. Because of these omissions, and because of possible small changes in the opacities in the relevant regions of the envelope, we must expect that all the numerical conclusions in this paper will be subject to some change which I hope will be small. However, the correlations that have been found should remain true in general form.

There is no indication in the examples that have so far been studied that the $\beta$ Cephei or $\beta$ Canis Majoris variables can be accounted for by the mechanisms responsible for RR Lyrae and Cepheid pulsation. The models appear to stabilize at $T_{e} \approx 7500^{\circ}$, and destabilization of the envelope to radial pulsation activated in the ionization zones at higher $T_{e}$ is not indicated. However, no survey of that problem has been made. On the other hand, as discussed in Paper I, it is very likely that similar mechanisms to those explored in this survey will account for pulsation in the long-period variables when a convective envelope can be properly treated.

The very interesting question of the ejection of matter from a pulsating variable by the action of the periodic shocks arising from pulsation has been only touched on in this paper. The techniques used here are not adequate to settle this question except where the ejection is very violent. We hope that future observations and calculations will be able to determine the amount of mass ejected by these mechanisms.

The author's work on variable stars was initiated while on sabbatical leave in 1961 at the Institute for Advanced Study, made possible by J. R. Oppenheimer and supported by the National Science Foundation.

The author would like to thank the many astronomers who have helped him with discussions of these problems; at the same time he would like to apologize for being unfamiliar with the very extensive literature in this field which has no doubt led to the omission of a number of important references. The assistance of the Computing Center at the California Institute of Technology in the extensive computations reported here is gratefully acknowledged. 


\section{APPENDIX}

THE OPACITY

The opacities used in these calculations were derived from the following formula:

$$
\begin{gathered}
\kappa=P_{e}\left[5.4 \times 10^{-13} \times V / T+X\left\{T^{1 / 2}\left(2 \times 10^{6} / T^{4}+2.1 \times T^{6}\right)^{-1}\right.\right. \\
\left.+\left[4.5 T^{6}+T^{-1}\left(4 \times 10^{-3} / T^{4}+2 \times 10^{-4} V^{1 / 4}\right)^{-1}\right]^{-1}\right\}+Y\left[\left(1.4 \times 10^{3} T+T^{6}\right)^{-1}\right. \\
\left.\left.+1.5\left(10^{6}+0.1 T^{6}\right)^{-1}\right]+Z\left[T^{1 / 2} \times\left(20 T+5 T^{4}+T^{5}\right)^{-1}\right]\right],
\end{gathered}
$$

where $T$ is the temperature in units of $10^{4}{ }^{\circ} \mathrm{K}, V$ is the specific volume, and $P_{e}$ is the electron pressure.

The basic source of the opacities used in deriving the expression above was a series of calculations carried out by A. N. Cox and J. N. Stewart (unpublished, but see Cox and Stewart [1965] for similar ones) at Los Alamos in the spring of 1962. I am very grateful to them for the use of these results prior to publication. These calculations included the effects of bound-bound transitions in addition to the usual bound-free, free-free, and scattering contributions. In the region of interest here, between $10^{4}$ and $10^{5}$, the line-broadening effects make the bound-bound contributions (primarily from hydrogen and helium) as large as, or larger than, all the others. These calculations were provided for the following five mixtures by weight:

I:

II:

III:

IV:

V:

$$
\begin{array}{lll}
X=0.90, & Y=0.06, & Z=0.04 ; \\
X=0.50, & Y=0.46, & Z=0.04 ; \\
X=0.90, & Y=0.08, & Z=0.02 ; \\
X=0.50, & Y=0.48, & Z=0.02 ; \\
X=0.996, & Y=0.0, & Z=0.004
\end{array}
$$

In view of the subsequent treatment, the exact heavy-element mixture chosen (more or less standard) is not relevant.

Because these calculations were not sufficiently detailed in the photospheric region, they were augmented by additional calculations ${ }^{6}$ of the continuum opacity excluding, however, boundbound transitions for temperatures from $3000^{\circ}$ to $30000^{\circ}$.

These two sources of opacities were still inadequate, as those calculated here could not be used for $T>10^{\circ}{ }^{\circ} \mathrm{K}$ because of the omission of line effects and because the Los Alamos opacities were for a quite restricted set of compositions. Since my intention was to make a survey of the effects of varying the composition, it was decided to attempt interpolation and extrapolation of the Los Alamos opacities and to connect these with our own.

The basic procedure was to start with $\kappa$ (the opacity in $\left.\mathrm{cm}^{2} / \mathrm{gm}\right) / P_{e}$ (the electron pressure) and to attempt to solve, at each temperature and density, the equations resulting from the assumption that

$$
\kappa / P_{e}=f_{1}(V, T) X+f_{2}(V, T) Y+f_{3}(V, T) Z
$$

to find $f_{1}, f_{2}$, and $f_{3}$. This assumption that the opacity is additive in $X, Y, Z$ is not basically correct but was used as a matter of convenience. It is probably acceptable as an interpolation method but not as an extrapolation method. Actually, only the first four mixtures were used for this purpose. They provided enough information for determining three quantities $\left(f_{1}, f_{2}, f_{3}\right)$ and one check. The check showed that these opacities could be reasonably so represented.

${ }^{6} \mathrm{I}$ am indebted to $\mathrm{J}$. Noble for assistance in these calculations. 
The resulting sets of $f_{1}, f_{2}, f_{3}$ for each $V, T$ were then represented approximately as algebraic expressions in $V, T$. In deriving these analytic expressions, we referred to integrations through a giant envelope to find the approximate paths in the $V, T$-plane of particular interest. The analytic approximations were then chosen to obtain a good fit to the opacities along these paths. This is a second stage of approximation from the original opacities. The reasons for this second approximation were various and not necessarily even valid. First, the original set of $V, T$ were so few in number that they would lead to a rather jumpy set of opacities which could interfere with the numerical iteration scheme (sometimes such an iteration will jump back and forth across a discontinuity and refuse to converge). Second, I had found from previous calculations that there was an inflection or bump in the opacity (as a function of $T$ ) near $4 \times 10^{4}$. This bump appears both in hydrogen and in helium and can be interpreted as a result of the peak of the Planck distribution coinciding with the ionization edge (Lyman edge in hydrogen). In helium a second such bump should appear at about $1.6 \times 10^{5}{ }^{\circ}$ associated with the second ionization edge of helium. Existing calculations are marginal to determine the first bump and inadequate to determine the second. In addition, there is the well-known peak near $2 \times 10^{4}$ 。 in the hydrogen opacity.

It was thought that some of these opacity peculiarities (in particular, that at $4 \times 10^{4}{ }^{\circ}$ which coincides with the second helium ionization) might be relevant in driving pulsation. I felt that they could be represented more adequately by carefully constructing curves to fit the limited number of opacities available but including effects leading to such bumps. These curves were then approximated by algebraic expressions.

Referring now to the opacity formula, we can see the purpose of the various terms. The first term just gives the free electron scattering contribution. In the remaining contributions, it was found that for the relatively low densities appropriate to giant envelopes, the opacity was approximately proportional to $\rho=1 / V$. This was then assumed in what follows. It limits the application of these expressions to regions where $\log V T>8$ (i.e., $V>10^{3}$ at $T=10^{5}$ ).

In the second term, due to hydrogen, the first term gives the opacity at high temperatures and peaks near $4 \times 10^{\circ}$. The second term takes over below $4 \times 10^{4}$ and goes into the lowtemperature region; this term has an additional $V$ dependence to rectify the fact that $\kappa / P_{e}$ is not $\propto \rho$ near $10^{4} \circ$.

In the third term, due to helium, the first term gives the low-temperature contribution and provides the break at $4 \times 10^{4}$. The last term comes in at $1.6 \times 10^{5}{ }^{\circ}$, giving a bump there.

The last term, relatively smooth, provides the heavy-element contribution.

This expression has been discussed in some detail, not because it is especially accurate but because, if any reader is interested in using the expression outside its range of validity, similar expressions but with adjusted form may be desired.

The present formula has been compared with the originals from which it was derived with the following result. The average $\log \kappa_{\text {formula }} / \kappa_{\text {original }}$ in the region above $2 \times 10^{4}{ }^{\circ}$ was calculated and gives an average error of 8 per cent in the density region appropriate to a giant envelope. This corresponds in this problem to $1 \leq \kappa \leq 100$. Compared to the nearly pure hydrogen mixture, the formula is about 30 per cent high.

Recently, Cox and Stewart (1965) have made available a series of opacity calculations for various element mixtures and including photospheric opacities. In addition, Vardya (1964) has published a series of photospheric opacities. I have therefore compared the formula with these new opacities.

In the photospheric region where hydrogen is the only significant opacity source, the formula gives results differing on the average by about 17 per cent from those of Vardya. Incidentally, the calculations of Cox and Stewart differ from those of Vardya also by about 17 per cent on the average. This comparison covers the region from $5000^{\circ}$ to $10000^{\circ}$ and $\rho=10^{-7}, 10^{-8}, 10^{-9}$, which is the important region for RR Lyrae and Cepheid variables.

In the region $4.4 \leq \log T \leq 5.8$, and densities so chosen that $\kappa$ is in the range $1-100$ which is appropriate for the kind of envelopes we are interested in, the results of the formula have been compared to recent calculations of Cox and Stewart with the following results. Compared to 
two population I mixtures, the mean error is 13 per cent; compared to a population II mixture, the error below $2 \times 10^{5}$ is only 5 per cent; above $2 \times 10^{5}$, the formula begins to fail since it gives opacities that are progressively too large. Apparently both the hydrogen and helium opacities above $2 \times 10^{5}$ are overestimated in my formula. Compared to pure helium opacities, the mean error is 45 per cent.

These new opacities of Cox and Stewart would provide the basis for significant improvement in the formula.

\section{REFERENCES}

Aleshin, V. I. 1964a, Astr. Zh., 41, 201. . $1964 b$, ibid., p. 1056.

Baker, N., and Kippenhahn, R. 1962, Zs.f. Ap., 54, 114. 1965, ibid., 62, 868.

Böhm-Vitense, E. 1958, Zs.f. Ap., 46, 108.

Christy, R. F. 1962, $A$ p. J., 136, 887 (Paper I).

- $1963 a$, A.J., 68, 275. 1963b, ibid., p. 534. 1964a, Rev. Mod. Phys., 36, 555 (Paper II). $1964 b, A . J ., 69,536$.

Cox, A. N., and Stewart, J. N. 1965, Ap. J. Suppl., 94, 1.

Cox, J. P. 1963, Ap.J., 138, 487.

Cox, J. P., and Giuli, R. T. 1961, Ap. J., 133, 755.

Cox, J. P., and Salpeter, E. E. 1961, Ap. J., 133, 764.

Cox, J. P., and Whitney, C. 1958, A p. J., 127, 561.

Dickens, R. J., and Saunders, J. 1965, R. Obs. Bull., No. 101.

Eggen, O. J. 1951, A p. J., 113, 367.

Härm, R., and Schwarzschild, M. 1964, $A$ p. J., 139, 594.

Hayashi, C., Hōshi, R., and Sugimoto, D. 1961, Prog. Theor. Phys., Suppl., 22, 1.

Hogg, H. S. 1955, Pub. David Dunlap Obs., 2, 35.

Kinman, T. D. 1959, M.N., 119, 538.

Kinman, T. D., Wirtanen, C. A., and Janes, K. A. 1965, Ap. J. Suppl., 100, 223.

Martin, W. Chr. 1938, Leiden Ann., 17, Pt. 2, 1.

Mihalas, D. 1965, Ap.J., 141, 564.

Oke, J. B., Giver, L. P., and Searle, L. 1962, $A$ p. J., 136, 393.

Oosterhoff, P. Th. 1943, B.A.N., 9, 399 (No. 356).

Preston, G. W. 1959, Ap. J., 130, 507.

Preston, G. W., and Paczyński, B. 1964, A p. J., 140, 181.

Preston, G. W., Smak, J., and Paczyński, B. 1965, A p. J. Suppl. 12, 99 (No. 104).

Roberts, M., and Sandage, A. 1955, A.J., 60, 185.

Sandage, A. 1958, Stellar Populations, ed. O'Connell (Rome: Recherche Astr. Specola Vaticana), Vol. 5.

Sandage, A., and Wallerstein, G. 1960, $A p$. $J ., 131,598$.

Schwarzschild, M. 1958, Structure and Evolution of the Stars (Princeton, N.J.: Princeton University Press).

Schwarzschild, M., and Härm, R. 1965, Ap. J., 142, 855.

Suda, K., and Virgopia, N. 1966, $A$ p. $J$., 143, 75, 87.

Tifft, W. G. 1964, Ap. J., 139, 451.

Vardya, M. S. 1964, Ap. J. Suppl., 80, 277.

Woolf, N. J. 1964, Ap. J., 139, 1081.

Zhevakin, S. A. 1963, Ann. Rev. of Astr. and Ap., ed. L. Goldberg, 1, 367. 